\title{
An Intelligent Telemedicine System for Detection of Diabetic Foot Complications
}
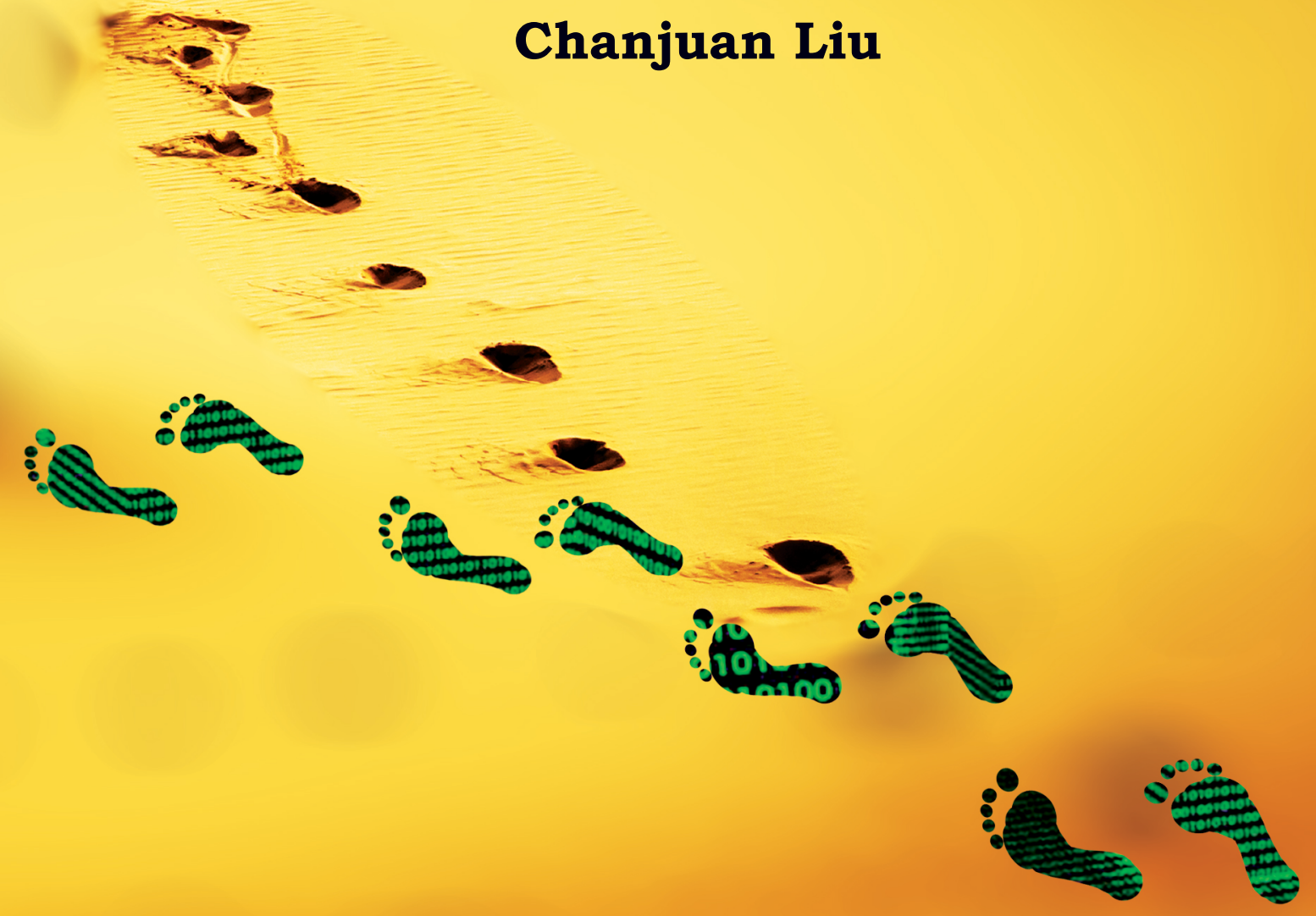


\section{An Intelligent Telemedicine System for Detection of Diabetic Foot Complications \\ Chanjuan Liu}


Graduation committee:

Chairman:

Promoter:

Assistant promoter:

Members:

Prof. dr. ir. P. H. Veltink

Prof. dr. ir. W. Steenbergen

Prof. dr. A. G. J. M. van Leeuwen

Referents:

Dr. J. G. van Baal

Dr. J. J. van Netten
Prof. dr. P. M. G. Apers

Prof. dr. ir. C. H. Slump

Dr. ir. F. van der Heijden

University of Twente

University of Twente

Academic Medical Center,

University of Amsterdam

Ziekenhuisgroep Twente Almelo

Ziekenhuisgroep Twente Almelo

This work is part of the project "Early detection of ulceration in diabetic feet an intelligent telemedicine monitoring system", which is funded by ZonMw.

Chair Robotics and Mechantronics - Medical Applications

EEMCS Faculty, University of Twente

P.O. Box 217, 7500 AE Enschede, The Netherlands

Copyright (C) Chanjuan Liu, Enschede, 2014

No part of this publication may be reproduced by print, photocopy or any other means without the permission of the copyright owner.

Printed by Gildeprint B.V., Enschede, The Netherlands

Typesetting in $\mathrm{LT}_{\mathrm{E}} \mathrm{X} 2 \varepsilon$

ISBN 978-90-365-3746-9

DOI 10.3990/1.9789036537469 


\title{
AN INTELLIGENT TELEMEDICINE SYSTEM FOR DETECTION OF DIABETIC FOOT COMPLICATIONS
}

\author{
DISSERTATION \\ to obtain \\ the degree of doctor at the University of Twente, \\ on the authority of the Rector Magnificus, \\ Prof. dr. H. Brinksma, \\ on account of the decision of the graduation committee, \\ to be publicly defended \\ on Friday 03 October, 2014, at 14:45 \\ by \\ Chanjuan Liu \\ born on 24 March, 1983 \\ in Shandong, China
}


This dissertation has been approved by:

Prof. dr. ir. C.H. Slump (promoter)

Dr. ir. F. van der Heijden (assistant-promoter) 


\section{Contents}

1 Introduction 1

1.1 Diabetic Foot Complications . . . . . . . . . . . . . . . . 1

1.2 Statement of the Problem . . . . . . . . . . . . . . . . . 3

1.3 Research Scope and Objectives . . . . . . . . . . . . 5

1.4 Thesis Outlining . . . . . . . . . . . . . . 6

2 Potential Modalities and Experimental Setup Development 7

2.1 Telemedicine on Diabetes Management . . . . . . . . . . 7

2.2 Potential Modalities . . . . . . . . . . . . . . . . . . . . . 9

2.3 Experimental Setup . . . . . . . . . . . . . . . . . . 16

2.4 Measurement Protocol . . . . . . . . . . . . . . . . . . . 17

3 Statistical Analysis of Spectral Data: a Methodology for Designing an Intelligent Monitoring System for the Diabetic Foot 23

3.1 Abstract . . . . . . . . . . . . . . . . 23

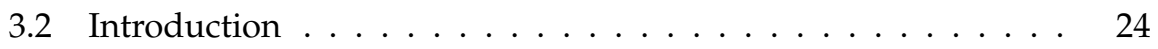

3.3 Collection of Training Data . . . . . . . . . . . . 26

3.4 Statistical Analysis for Selection of the Optical Filters . . . . . 29

3.5 Stability Analysis . . . . . . . . . . . . . . . . . . 42

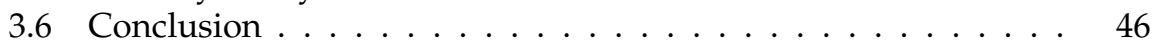

4 A Front end for Automatic Detection of Diabetic Foot Complications using Spectral Images: a Statistical Approach to Pixel Based Segmentation 49

4.1 Abstract . . . . . . . . . . . . . . . . . . 49

4.2 Introduction . . . . . . . . . . . . . . . . 50

4.3 Materials and Image Acquisition . . . . . . . . . . . . . 53

4.4 Spectral Image Processing and the Design of the Pixel Classifier 56 
4.5 Results and Discussion . . . . . . . . . . . . . . 72

4.6 Conclusion . . . . . . . . . . . . . . . . 86

5 Automatic Detection of Diabetic Foot Complications with Infrared $\begin{array}{lr}\text { Thermography by Asymmetric Analysis } & 89\end{array}$

5.1 Abstract ....................... 89

5.2 Introduction . . . . . . . . . . . . . . . . . . . . . . . . . . . .

5.3 Materials and Measurements . . . . . . . . . . . . . . 93

5.4 Methodology for Automatic Detection of Diabetic Foot Compli-

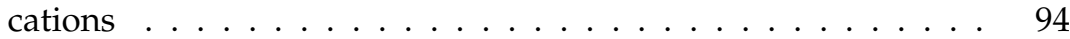

5.5 Results and Discussion . . . . . . . . . . . . . . . . 105

5.6 Conclusion . . . . . . . . . . . . . . . . . . 111

6 Photometric Stereo Imaging to Reconstruct the 3D Surface of the Plantar Foot of Diabetic Patients: A Feasibility Study on Detection of Diabetic Foot Complications

6.1 Abstract ......................... 113

6.2 Introduction . . . . . . . . . . . . . . . . . . . . 114

6.3 Photometric Stereo Imaging . . . . . . . . . . . . . . . . . . . . 116

6.4 Materials and Measurement . . . . . . . . . . . . . . . . 117

6.5 Method . . . . . . . . . . . . . . . . . . . . . 118

6.6 Results and Discussion . . . . . . . . . . . . . . . . . 122

6.7 Conclusion . . . . . . . . . . . . . . . . . . 125

$\begin{array}{lll}7 \text { Conclusion } & 127\end{array}$

7.1 Answers to Research Questions . . . . . . . . . . . . . . . . . . 127

7.2 Recommendations for Future Work . . . . . . . . . . . . 130

$\begin{array}{ll}\text { A Live Assessment Form } & 135\end{array}$

$\begin{array}{ll}\text { Bibliography } & 139\end{array}$

$\begin{array}{ll}\text { List of publications } & 151\end{array}$

$\begin{array}{ll}\text { Summary } & 153\end{array}$

$\begin{array}{ll}\text { Samenvatting } & 155\end{array}$

$\begin{array}{lr}\text { Acknowledgments } & 157\end{array}$ 


\section{Introduction}

\subsection{Diabetic Foot Complications}

Diabetes Mellitus (DM) is one of the most common chronic diseases over the world, and it continues to increase in population and significance. There were 194 million people suffering from DM worldwide in 2004 [1], and this number is expected to grow to 439 million by 2030 due to longer life-expectancy and changes in dietary habits [2]. People with diabetes are at risk of developing a number of disabling and life-threatening health problems, such as lower limb amputation, blindness and kidney failure [2]. Diabetes management requires frequent assessment, continuous medical care, and ongoing self-management education and support to avoid the potential consequences from mismanagement of diabetes [2-7]. All of these cause a great burden for the patients themselves and the society. While the prevalence of DM and its complications expand, the economy regarding the health care sector is facing notable challenges as well [3]. Taking the year of 2013 as an example, about 382 million people worldwide have diabetes; among them, 5.1 million died from DM, and above 548 billion US dollars have been spent in treating diabetes and manage the complications globally [8].

Many patients with DM are affected by vascular and neurological complications in the lower extremities. These conditions significantly increase the risk of developing diabetic foot ulceration (DFU), which is one of the major diabetic foot complications. DFUs result from complex reactions of numerous factors $[3,4,9]$. The causal pathways to DFU have been illustrated in Fig. 1.1. Approximately, $15 \%$ to $25 \%$ of patients with DM eventually develop DFU, with a yearly incidence of $2 \%[4,8,10]$.

With the protective layer of the skin broken, DFUs provide an avenue for bacterial colonisation. If not adequately treated, these ulcers may lead to 


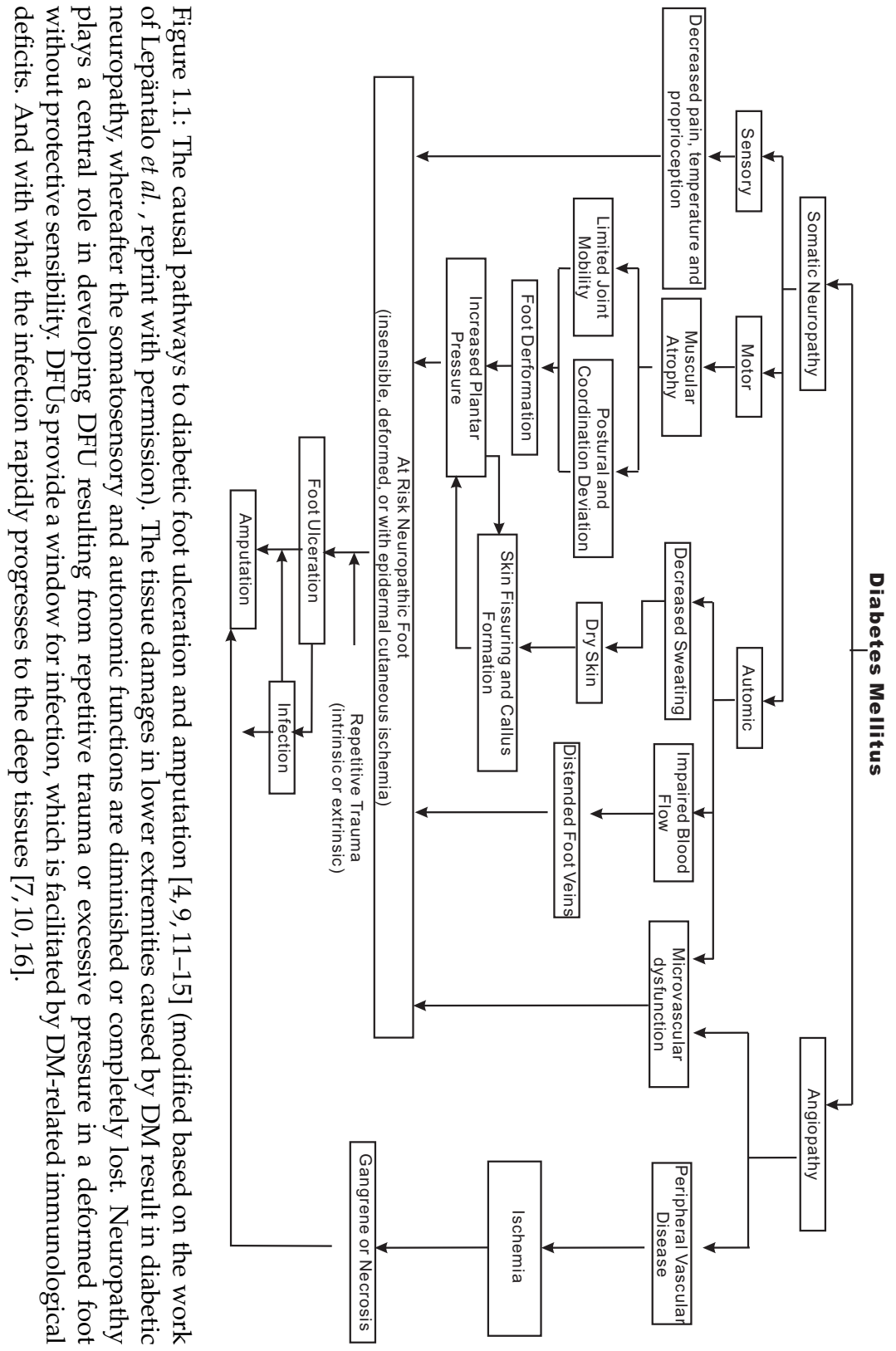


foot infection, and ultimately to total (or partial) lower extremity amputation, which imposes a heavy burden on both the patient and the society [3,8]. It has been shown that DFUs are the leading causes for hospitalisation and lower extremity amputations; above $85 \%$ of all lower extremity amputations in patients with DM resulted from foot ulcerations [17]. Thus, prevention of DFU is one of the foci of any amputation prevention program $[5,6]$. Researchers and practitioners are challenged to find efficient and effective solutions to avoid DFU and the amputation.

Currently, risk assessment and status monitoring of feet of patients with DM are performed routinely in many countries, following various publications of clinical guidelines for DFU management $[4,7,18]$. Risk identification, education, proper foot care, multidisciplinary treatment, prevision of appropriate footwear and close monitoring are fundamental of DFU management and can help to reduce the amputation rates by $49-85 \%[3,11]$. However, Lavery et al. pointed out that the incidence of onset of DFUs and lower amputation can be further reduced $[5,6]$.

\subsection{Statement of the Problem}

The onset of diabetic foot ulcers may be preventable in case of early identification and subsequent treatment of ulceration and its pre-signs, such as callus formation, redness, fissures, and blisters (Examples of different diabetic foot complications are illustrated in Fig. 1.2). This early identification strongly depends on frequent risk assessment, preferably on a daily basis especially for high-risk patients [7]. However, frequent assessment by healthcare professionals is costly and not always possible. Due to the complications from DM (e.g. limited joint mobility and bad eyesight), self-examination by patients themselves are difficult and impractical.

Thus, any non-invasive, non-interactive and user-friendly initiative, which may overcome these limitations and contribute to automated detection of early warning signs, should be supported and implemented in diabetic feet care [6]. A solution would be to apply an automated monitoring system that is easily accessible for the patient. Problems are then: a) what should be the principle of operation of such a device, $b$ ) how should this device be operated such that it is easily accessible for the patient (e.g. the ergonomics of the device), and c) how should this device put to service within the system of health care (e.g. the business model, the servicing model, etc.). The current study focusses solely on the first aspect: the principle of operation. 
1 Introduction

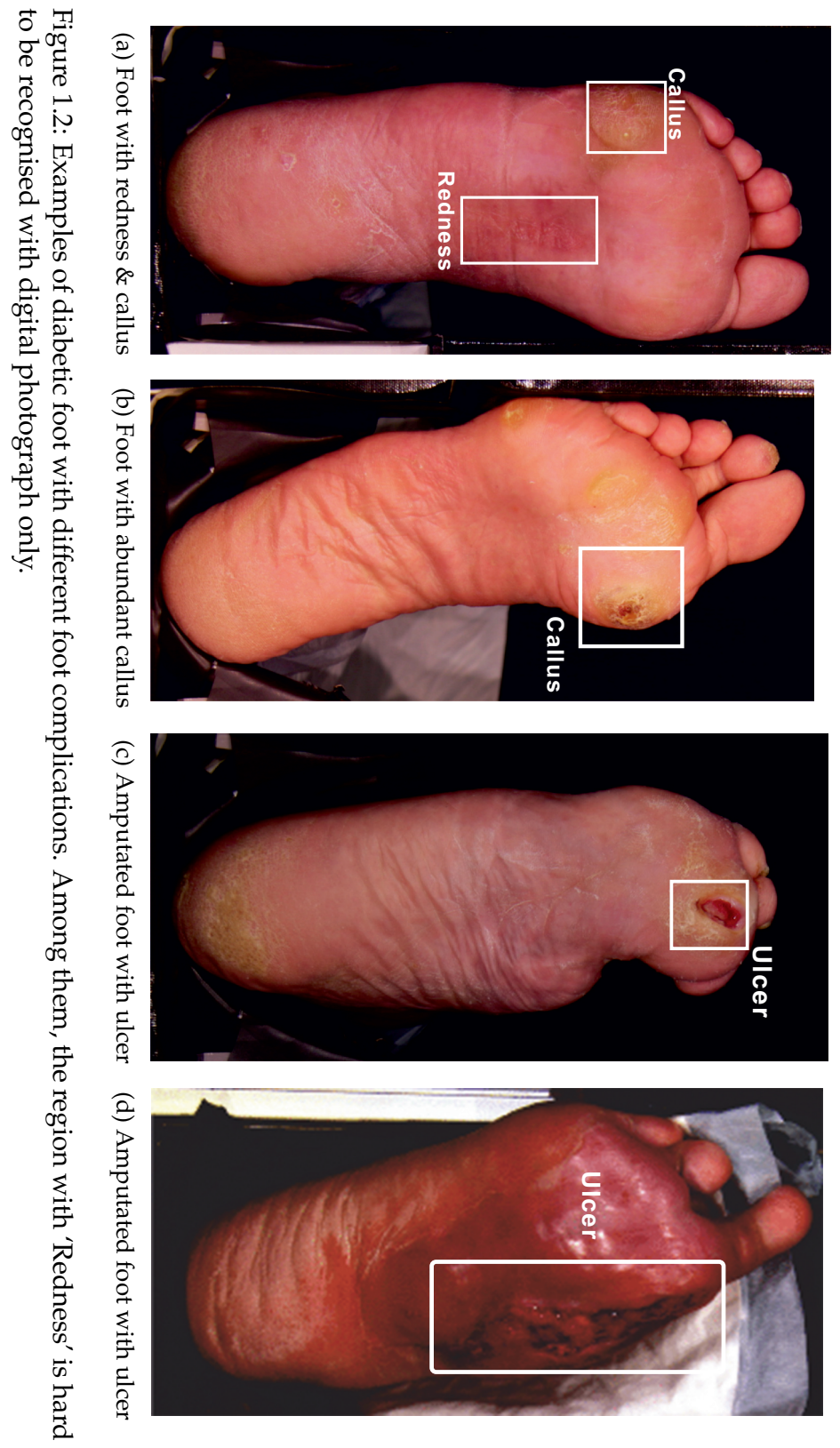




\subsection{Research Scope and Objectives}

In this thesis, our goal is to develop and investigate the technologies for building an intelligent telemedicine monitoring system that can be deployed at the patients' home environment or general practitioner's place for frequent examination of the patients' feet to detect diabetic foot complications (ulceration and its pre-signs, such as callus formation, redness, fissures, and blisters) in a timely manner.

To achieve this goal we first invested the feasibility of telemedicine systems for diabetic foot complications, compared potential technologies and made a pre-selection based on this literature study. An experimental setup with three modalities, which are Spectral Imaging, Thermal imaging and Photometric Stereo Imaging, has been built and is being used for data collection on patient at high risk for developing DFU and amputation.

Based on the acquired patients' data, we addressed the following research questions:

- Can skin spectra be used to detect diabetic foot complications, and to distinguish different foot complications?

- What are the most informative wavelength bands for the detection of diabetic foot complications and for distinguishing the different diabetic foot complications? Is it possible to build a Spectral Imaging system that employs these informative bands?

- Can the spectral imaging system with the selected bandpass filters designed in our experimental setup detect different diabetic foot complications? What is the performance?

- Can infrared thermal imaging system in our experimental setup can be used for the detection of some of the diabetic foot complications?

- What kind of information can photometric stereo imaging provide in their application? Can photometric stereo imaging be used for the detection of diabetic foot complications? 


\subsection{Thesis Outlining}

The remainder of the thesis is organised as follows:

Chapter 2 gives an overview of current state and art of the technologies in the telemedicine systems for diabetic foot complications. A pre-selection of three modalities is made based on the investigation, which are Spectral Imaging, Thermal imaging and Photometric Stereo Imaging. Integrated with the three modalities, an experimental setup has been developed and has been used for data collection in hospital on a group of high-risk diabetic patients.

Chapter 3 describes the design of a spectral imaging system with a small number of selected optical bandpass filters for detecting and discriminating different diabetic foot complications. The filter selection is done with features selection technologies on the data acquired from foot spots using a spectrometer.

Chapter 4 employs the designed spectral imaging system to acquire spectral images at discrete wavelengths to detect diabetic foot complications. Supervised machine learning technology was employed for the detection. A classifier was trained with spectral data from spectral images, with true labels from annotation from clinical experts.

Chapter 5 compares the temperature difference between corresponding areas on the left and right foot to detect the risk of inflammation. This was done with the following steps: thermal foot segmentation with assistance from digital color images, left and right foot registration with non-rigid B-splines registration, and high-temperature-difference risk detection by overlapping the registered left and right foot.

Chapter 6 addresses the possibility to find information on diabetic foot complications using the three dimensional height map reconstruction and skin albeldo reconstruction from the photometric stereo images set.

Chapter 7 concludes this thesis by summarising the research results and providing recommendations for further work. 


\section{Potential Modalities and Experimental Setup Development}

\subsection{Telemedicine on Diabetes Management}

Telemedicine (TM) systems are more and more attention. They are building up huge expectations in various medical specialities, as they address several major challenges: a) to improve the accessibility of healthcare, especially for patients in underserved or remote areas; $b$ ) to increase the frequency of assessment; $c$ ) to provide a solution to the scarcity of clinical professionals dealing with epidemic diseases; $d$ ) to reduce the cost of healthcare while improving the quality $[19,20]$.

The attempts to leverage of TM system on diabetes management date back to late 1970s for self-monitoring blood glucose. With four decades development and investigation, researchers concluded that a proper implemented TM system is safe and sounding for managing patients with DM [21-23]. TM has also been of particular interest in monitoring diabetic foot complications. Several such applications have been developed, which can be summarised into the following categories:

Tele-consultation through Cellular Phone or Video Conference [24-27]

With the emergence of Universal Mobile Telephone System (UMTS) and the rapid development of smartphone, tele-consultation through a cellular phone, with audio or video communication, gained more attention in the area of telemedicine system on diabetes management. With such a system, the patients and healthcare workers (e.g. visiting nurse) are linked with clinical specialist. The acquired data by the healthcare workers, such as notes, audio and video are transmitted to a clinical center server for archiving with other electrical medical data. These data will be downloaded and reviewed by clinical 
specialists. As such, a real-time diagnostic feedback is feasible. Studies imply that the treatment of DFU may benefit from such systems, where patients are allowed to stay at home, thereby decreasing the transportation and waiting time for an in-person consultation. The cooperation between the visiting healthcare workers and clinical specialist may increase the treatment quality $[26,27]$.

\section{Remote assessment by three-dimensional (3D) wound imaging [28, 29]}

Bowling et al. $[28,29]$ have developed a digital optical system that creates a 3D image of the ulcer region to measure the wound length, depth, volume, surface area and surface curvature. The authors concluded from the pilot studies that the measurement obtained from this system showed promising outcomes in predicting ulcer healing and providing information to guide the DFU treatment. However, no statistical meaningful conclusion could be drawn with the small sample size in the initial studies [29].

\section{Remote diagnostic with digital photography [30-33]}

Telemedicine devices with digital photography imaging sensors have been designed and used to monitor the diabetic foot in the home environment [30-33]. Four clinical observers (two wound specialist and two surgeons) participated in the live assessment and the assessment based on the photographic recordings. To avoid bias from memory, the photographic assessment was repeated two weeks and again four weeks post imaging. Studies show that a good agreement between the live and photographic assessment and between repeated photographic assessment could be obtained for diagnosis of different (pre-)signs of ulceration, such as presence of ulceration and abundant callus $[30,31]$. The authors also indicated a good feasibility of the utilisation of the photographic imaging device in the patients' home environment [32]. However, inflammation and infection, which are vicarious markers of diabetic foot complications, are difficult to assess using digital photography [32]. This was further elaborated by combining digital photographs with thermographic measurements to diagnose of diabetic foot infections. Such combination showed promising results [33].

\section{Wound area measurement with an Optical Scanner [34-36]}

Similar studies were conducted by another group of researchers to monitor the wound area through home-usage of a telemedicine system (TeleDiaFos). This system employed an optical scanner instead of digital cameras, to assure good lightening conditions and to avoid extra effort for developing a stand and a customised foot support for the device. Besides of the scan recordings, other 
medical data (blood glycymia and blood pressure) were also stored and transmitted to the clinical centre service to help the clinical specialists in evaluating the wound healing process [34-36]. By projection of a curved skin surface (foot plantar) to a flat scanner screen, there were some limitations that could not be avoided, such as the effect of the projection angle in the wound measurement. Although a disinfection procedure was involved for this TM system, it is still not suitable for all vulnerable diabetic feet with wounds as a contact device. Even with these limitations, patients found the device easy to use and it gave them a sense of safeness [36].

These TM systems show great potential in expanding the healthcare geometrically, especially for patients in the rural areas. They all act as bridge to link the patients remotely to the clinical specialists. However, non of them are yet capable of automatic detection and diagnosis of diabetic foot complications, which means they still require significant time and effort from healthcare professionals. An extra organisation of the healthcare professionals is required. This limits the application and implementation of these above mentioned TM systems.

Thus, there is still a distinct need for a non-invasive, non- interactive, more comprehensive and intelligent TM system, in which several technologies are integrated in order to detect and to discriminate different diabetic foot complications (such as ulceration, callus, fissure, redness, etc.), and to work more efficient in prevention, monitoring or treatment of diabetic foot complications.

The goal of the study presented in this thesis is to design and develop the technologies that may be used to build such an intelligent TM system.

\subsection{Potential Modalities}

The predecessor of this study, Vincent 50 [30-32], showed that some of the important pre-signs of ulceration, such as blister and redness, can hardly be diagnosed using digital photography only. Besides, complimentary preliminary research $[37,38]$ noticed that changes of the appearance of a risk area based on digital photography does not automatically imply the beginning of an ulcer. The tentative conclusion from Vincent 50 was that the photographic images do not carry sufficient information for reliable, automated diagnosis of foot disease in diabetic patients and that more sophisticated technologies are required. As successor of Vincent 50, we proposed to use multiple imaging modalities, instead of plain photographic imaging, to automatically detect different diabetic 


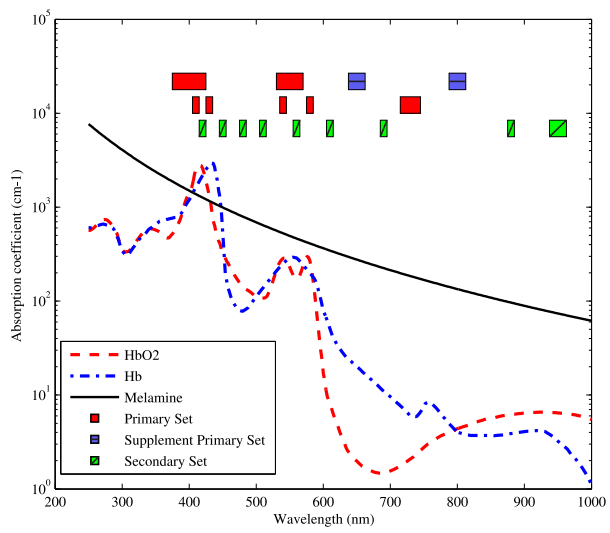

Figure 2.1: The absorption spectrum of main chromophores in skin [39]. $\mathrm{HbO}_{2}$ absorption features a strong maximum around $412 \mathrm{~nm}$, and maxima at $542 \mathrm{~nm}$ and $577 \mathrm{~nm}$, while $\mathrm{Hb}$ exhibits peaks at $430 \mathrm{~nm}$ and $555 \mathrm{~nm}$. The rectangles in the plot correspond to the distributions of the filters in two filter sets of our experimental setup. The red rectangle in the primary set are the optical filters selected [40]. The two blue supplement filters in the primary set, and the green ones in the second filter set were selected by visual inspection of the absorption spectra of $\mathrm{HbO}_{2}$ and $\mathrm{Hb}$, when there is large difference or equal value between these two spectra.

foot complications.

Besides of the TM system intended for home-use introduced above, there are also other technologies that have been applied in the area of prediction of DFU and/or monitoring the wound healing. Examples of these modalities are (hyper)spectral imaging and thermal imaging. Beyond these two, 3D reconstruction can also be a possible modality for the desired system, for it gives a more realistic, geometrical representation of the patents' foot. A detailed introduction of the possible modalities can be found below.

\subsubsection{Spectral Imaging}

Spectral imaging is a promising technology for our desired TM system, as it provides a hybrid modality for optical diagnosis with spectral data of the entire area under measurement and rendered in image form [41-44]. 


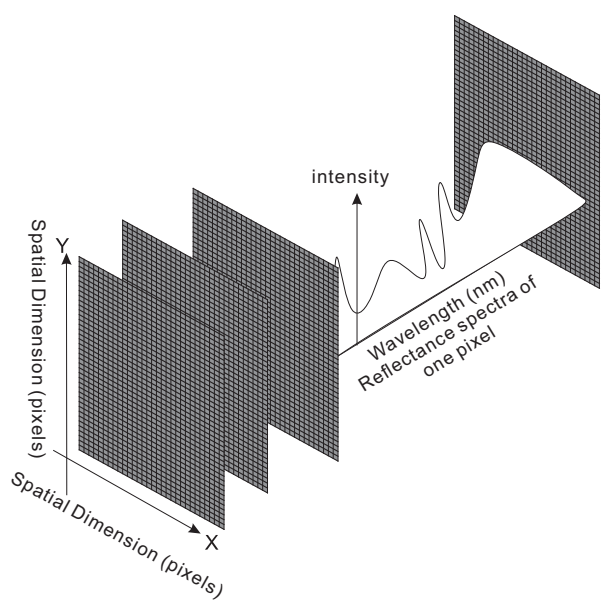

Figure 2.2: Hyperspectral Imaging generates a three dimensional dataset, which is called hypercube $I\left(x, y, \lambda_{i}\right)$, where $x$ and $y$ are two spatial dimensions and $\lambda$ presents the spectral dimension.

\section{Skin Optics - Basis for Spectroscopy as a Diagnostic Tool}

The spectral properties of tissue are determined by the interaction of light with human tissue [45-47]. The light incident onto human skin may be absorbed by skin chromophores. It may also be scattered by the cells or collagen fibers throughout the skin layers. The main chromophores of human skin are melanin in the epidermis layer, and oxy-haemoglobin $\left(\mathrm{HbO}_{2}\right)$ and deoxyhaemoglobin $(\mathrm{Hb})^{1}$ in the dermis layer. Different chromophores have different absorption spectra, as shown in Fig 2.1. Any small changes in the distribution of these skin chromophores may result in obvious changes in skin spectral reflectance. Besides, tissue structures and contents, such as thickness of the epidermis layer, determine the formation of the scattered diffuse reflection, which also accounts for changes in spectral reflection [45-47].

Impairments in microcirculation and macrocirculation of the diabetic foot play an important role in the development of diabetic foot ulcerations and the subsequent failure to heal $[4,9,12-15,48]$. Initial studies found that reduced oxygen supply might be the main reason for the development of the tissue

\footnotetext{
${ }^{1}$ Haemoglobin is the molecule in blood responsible for oxygen transportation from lungs to the rest of the body. It has two forms, which are oxygen bounded, $\mathrm{HbO}_{2}$, and oxygen unbounded, $\mathrm{Hb}$.
} 
hypoxia and nerve dysfunction [49].

These form the basis for spectroscopy to visualise the oxygen saturation and tissue structure in the perfusion of blood of diabetic feet, to detect, to predict or to monitor the development/healing of DFU.

Pilot studies on the skin's optical properties with spectroscopy (measurement on specific points) from diabetic human or animal samples indicated that the concentration of $\mathrm{Hb}$ can be 8 times higher than that of $\mathrm{HbO}_{2}$ in the diabetic tissue, which might account for the reduced oxygen supply; and the scattering is also higher for the diabetic tissue for its inhomogeneity [50-54].

\section{Spectral Imaging On Diabetic Foot}

Original developed by Department of Defence (DoD) for military purpose, combining spectroscopy and photographic imaging, hyperspectral imaging has been used for decades to map the earth surface to discriminate between different soil types or different architectures. Recently, (hyper-/multi-)spectral imaging has emerged as a new modality in medical imaging techniques to provide a powerful diagnostic tool for non-invasive and non-contact tissue analysis. Generally, spectral imaging systems consist of wide band illumination sources and a number of optical filters, which help to record images at a number of discrete wavelengths. Each pixel of the acquired hypercube corresponds to the local reflectance spectrum of the target, as shown in Fig. 2.2.

Several studies have been conducted to implement hyperspectral imaging for monitoring diabetic neuropathy [55], for monitoring and predicting ulcer healing process [56-58], and for assessing the risk for ulceration formation [59]. Beyond these three options for diabetic foot management, an intelligent monitoring system should also be capable of automatic recognition of ulcers and its pre-signs and to be able to discriminate these complications from the healthy skin. This may be achieved by statistical analysis to signify the difference between spectral data from healthy skin and skin with diabetic foot complications.

However, spectral imaging is currently still at the experimental stage, and hard to be implemented in a home environment due to its high cost.

\subsubsection{Thermal Imaging}

Inflammation plays a central role in the occurrence of two of the most devastating diabetic foot complications, which are DFU and Charcot's Foot, while 
increased plantar foot temperature is one of the main indicators of inflammation [60-65]. Monitoring the temperature changes in diabetic foot frequently can be significantly useful in diabetic foot management with the intent to determine the risk of DFU $[5,6,63-71]$.

Clinical studies on the home-monitoring of plantar foot temperature with an infrared thermometer (measurement done on six specific points on each foot) have shown that frequent temperature assessments and treatment in case of temperature differences $>2.2{ }^{\circ} \mathrm{C}$ between a foot region and the same region on the contralateral foot, can prevent diabetic foot complications [5,6]. It also has shown clinically that temperature assessment of the plantar foot are not only usefully for preventing DFU recurring but also may be indicative of subclinical neuropathy and assess the wound healing trajectory $[65,72]$. As such, thermography is a prime modality for an intelligent telemedicine monitoring system.

The technologies for temperature measurement of the foot plantar in diabetic foot management fall in three categories: hand-held dermal infrared (IR) thermometers [5,6,63] (Fig.2.3a), IR camera systems (Fig.2.3b)[67, 68,70,71,73, $74]$, and liquid crystal thermography (LCT, as shown in Fig. 2.3c) [66, 69].

The shortcoming of the hand-held dermal IR thermometers in the home environment is that the temperature is measured manually on specific spots on the foot. This makes it subjective, and it is impossible to obtain the temperature distribution of the whole foot.

Compared with LCT, IR camera systems have the following advantages:

No extra illumination sources are necessary IR camera systems measure the radiant heat emission from the target object. This may reduce the complexity of the system. On the other hand, LCT requests white light in the visible range, no ultraviolet (UV) or IR light involved, as UV light may cause the the sensors compounds to degrade and IR light may result in radiant heating effect on the surface.

Contactless measurements This prevents unwanted pressures and the transmission of pathological organisms, and hence avoids surface temperature changes due to the measurements [71,72]. As a curved surface, part of regions on foot plantar, such as the medial arch, can be easily measured by IR camera systems but hardly by LCT plate. Additionally, it is capable of measuring the dorsal side of the foot as well.

As such, IR camera systems show greater potential for telemedical applications and they will be the focus of this study. 


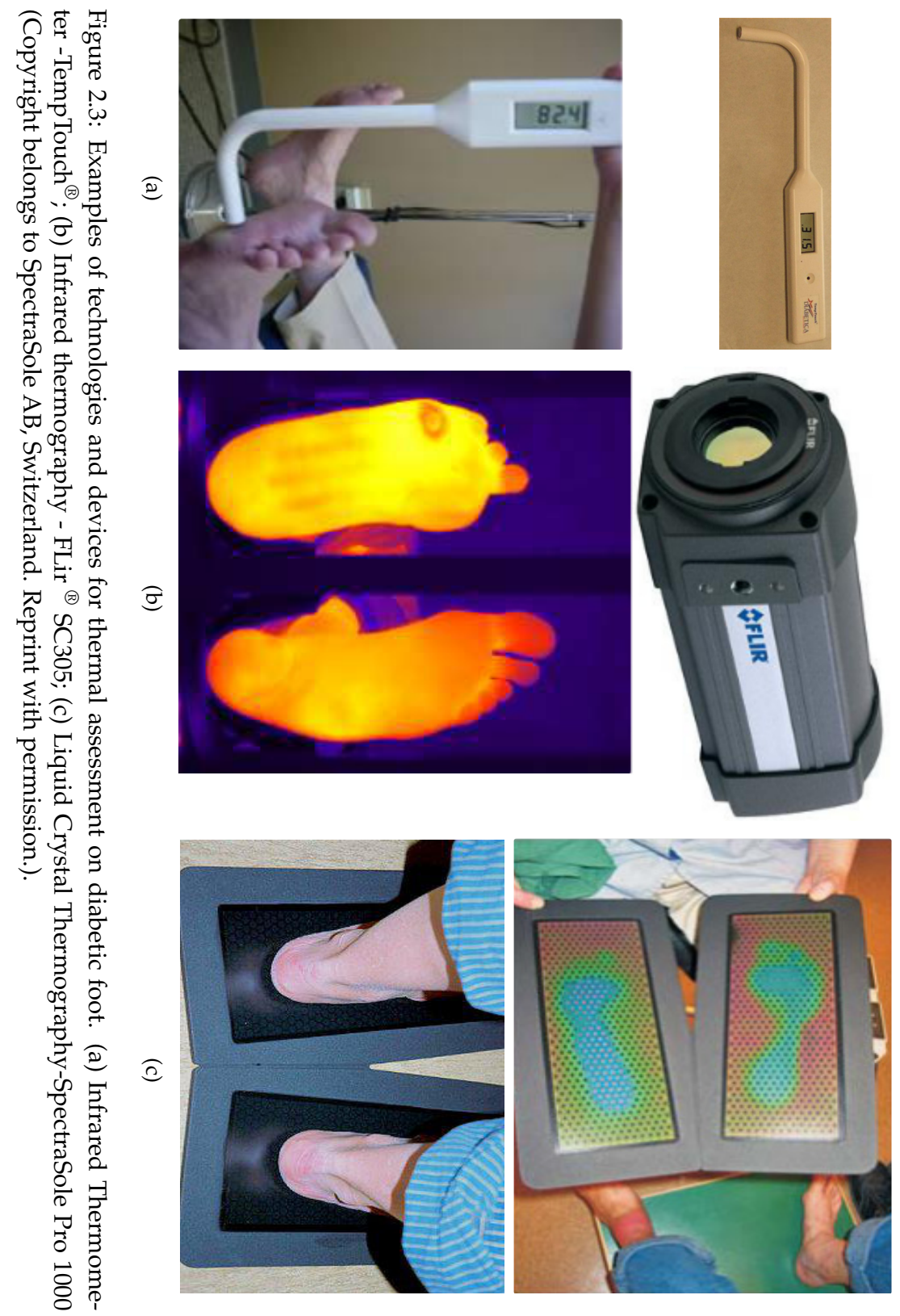




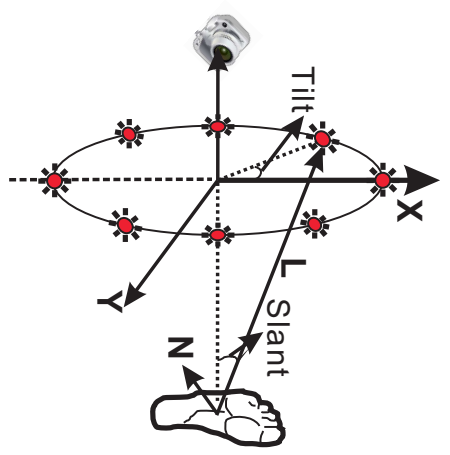

Figure 2.4: Schematic photometric stereo imaging setup with one camera and a number of illumination source. $\mathbf{L}$ is the illumination and $\mathbf{N}$ is the surface normal.

\subsubsection{D Surface Reconstruction}

3D shape acquisition and reconstruction is a beneficial but challenging problem in many fields of engineering and life science. The 3D geometry of the foot surface may be useful for revealing the local 3D surface deformation of the foot sole skin surface, which then provides information about changes in the surface textures. Such changes are either caused by geometrical variation of the surface (e.g. papillary lines, or pre-signs of ulceration such as fissures, abundant callus) or by radiometrical variations (e.g. necrosis, infection related redness, or an ulcer). We envision that diabetic foot complications can be detected by the usage of such 3D texture changes.

Numerous 3D scanning technologies exist. They generally fall into three categories, which are multi-view imaging, photometric stereo imaging (PSI) and tactile imaging. Multi-view imaging techniques often require elaborate setups [75], and focus more on the global 3D shape reconstruction, and thereby failing to capture high-frequency structure details [76]. Tactile imaging systems measure the local pressure on the object surface and they translate the measured pressure to a 3D surface [77]. The pressure on the foot skin is fundamental for tactile imaging, but may cause the transmission of pathological organisms in our application. PSI, on the other hand, is a modest and noncontact technology, which achieves the 3D surface reconstruction based on a set of images taken from one fixed viewpoint under three or more different illumination conditions [78-82]. The schematic of a PSI system is illustrated in Fig. 2.4. In PSI, the low spatial frequency components of measured gradi- 
ents are rejected. 3D surfaces are derived based on higher spatial frequency components, which can capture the local surface details.

As such, PSI shows greater potential for future telemedicine applications and was chosen for the developing our experimental setup.

\subsection{Experimental Setup}

An experimental setup has been built that Integrates the three imaging modalities, namely, spectral imaging, infrared thermal imaging and photometric stereo imaging. It has been used for data collection on patients at high-risk for amputation and with presence of diabetic foot complications. Measurements and data collection have been done in the hospital (Ziekenhuisgroep Twente, Almelo, the Netherlands). The illustration of the experimental setup can be found in Fig. 2.5.

\section{Spectral Imaging (SI )}

For spectral imaging (SI), an imaging system consists of an array of nine cameras, four illumination sources and two optical filter arrays (nine filters each), has been developed. Eight wide-band Quartz-Tungsten Halogen (QTH) lamps (400 $\sim 1000 \mathrm{~nm}$ ) were mounted with equal distance to each other at the boarders of a square (Fig. 2.6) at a distance of $465 \mathrm{~mm}$ from the target plane, which provide quasi-diffuse illumination with sufficient intensity at all wavelength. The two sets of the illustration sources, for PSI and for SI, are placed at delicate distance to the object and to each other, to make sure that there are no interaction and affects with each other when illumination illuminating the patients' feet.

To eliminate the image acquisition time and avoid the mechanical vibration, a camera array structure was employed: $3 \times 3$ matrix ( Fig. 2.6b) consisting of 9 monochrome cameras (GigE Flea 3, Point Grey, Ludwigsburg, Germany) that can perform image acquisition simultaneously. The cameras were all fitted with Schneider Kreuznach Xenoplan 1.4/17 lenses (Bad Kreuznach, Germany), at a distance of $1240 \mathrm{~mm}$ from the measurement plane. The diaphragm of the lenses were set to F4.0 and fixed. Each camera covered a field of view $490 \times 370$ $\mathrm{mm}^{2}$, with $1600 \times 1200$ pixels $(1$ pixel $\approx 0.3 \mathrm{~mm}$ ). The distribution of spectra coverage of the filters are illustrated in Fig. 2.1, which were chosen based on the absorption spectra of $\mathrm{Hb}$ and $\mathrm{HbO}_{2}$ and the study in Chapter 3.

\section{Infrared Thermal Imaging (TI)}

This modality in our experimental setup consists of one IR camera, FLIR SC305 
(Flir Systems GmbH, Frankfurt am Main, Germany).The IR camera has a resolution of $320 \times 240$ pixels and is placed at a distance of $860 \mathrm{~mm}$ from the measurement plane, covering a field of view of $400 \times 350 \mathrm{~mm}^{2}$. It provides a pixel distance on the foot soles of about $1.25 \mathrm{~mm}$. Besides, a set of six thermal reference elements are mounted in the field-of-view of the thermal camera, 4 elements above and 2 below the foot positions. The top surfaces of the reference elements are sandblasted and coated with flat black paint in order to provide maximum infrared emissivity. The elements are designed to provide a homogeneous temperature distribution over their entire top surfaces. Prior to applying the experimental setup for any clinical tests, the uniformity of the IR camera response was characterised with the help of this six thermal references. For calibration, these elements were heated to different, accurately known, constant temperatures in the expected temperature range for foot soles $\left(20^{\circ} \mathrm{C}-38^{\circ} \mathrm{C}\right)$ and serve as long-term reference of the absolute temperatures.

Through calibration, the maximal difference of temperature readings of the camera of two isothermal references, one far left and one far right in the image plane, is $\pm 0.25^{\circ} \mathrm{C}$ for $4 \times 4$ pixel areas (circa $5 \times 5 \mathrm{~mm}^{2}$ ).

\section{Photometric Stereo Imaging (PSI )}

The PSI modality in this experimental setup conducts the image acquisition by the corporation of one commercial digital RGB camera, Canon EOS 40D, and eight power LED light sources [83] as shown in Fig. 2.5 and Fig. 2.6. The digital camera was placed at a distance of $860 \mathrm{~mm}$ from the measurement plane, which is at the same distance of the IR camera and covering a field of view $420 \times 280 \mathrm{~mm}^{2}$ and acquiring images with resolution $3888 \times 2592$ pixels (pixel size $\approx 0.1 \mathrm{~mm}$ ). The eight illumination sources were mounted on a circle $(\phi \approx 565 \mathrm{~mm})$ with equally spaced tilt angles. The distance between the target imaging plane and the illumination plane was $480 \mathrm{~mm}$. As such, the slant angles for each illumination sources were about the same, $\approx 30^{\circ}$, satisfying the requirement for photometric stereo imaging system design [80].

\subsection{Measurement Protocol}

The measurement and research proposal with the above experimental setup has been approved by Medical Ethical Committee of Twente (METC Twente). The patients were recruited for this study from the multidisciplinary diabetic foot clinic of the Hospital Group Twente, Almelo, the Netherlands. Before 


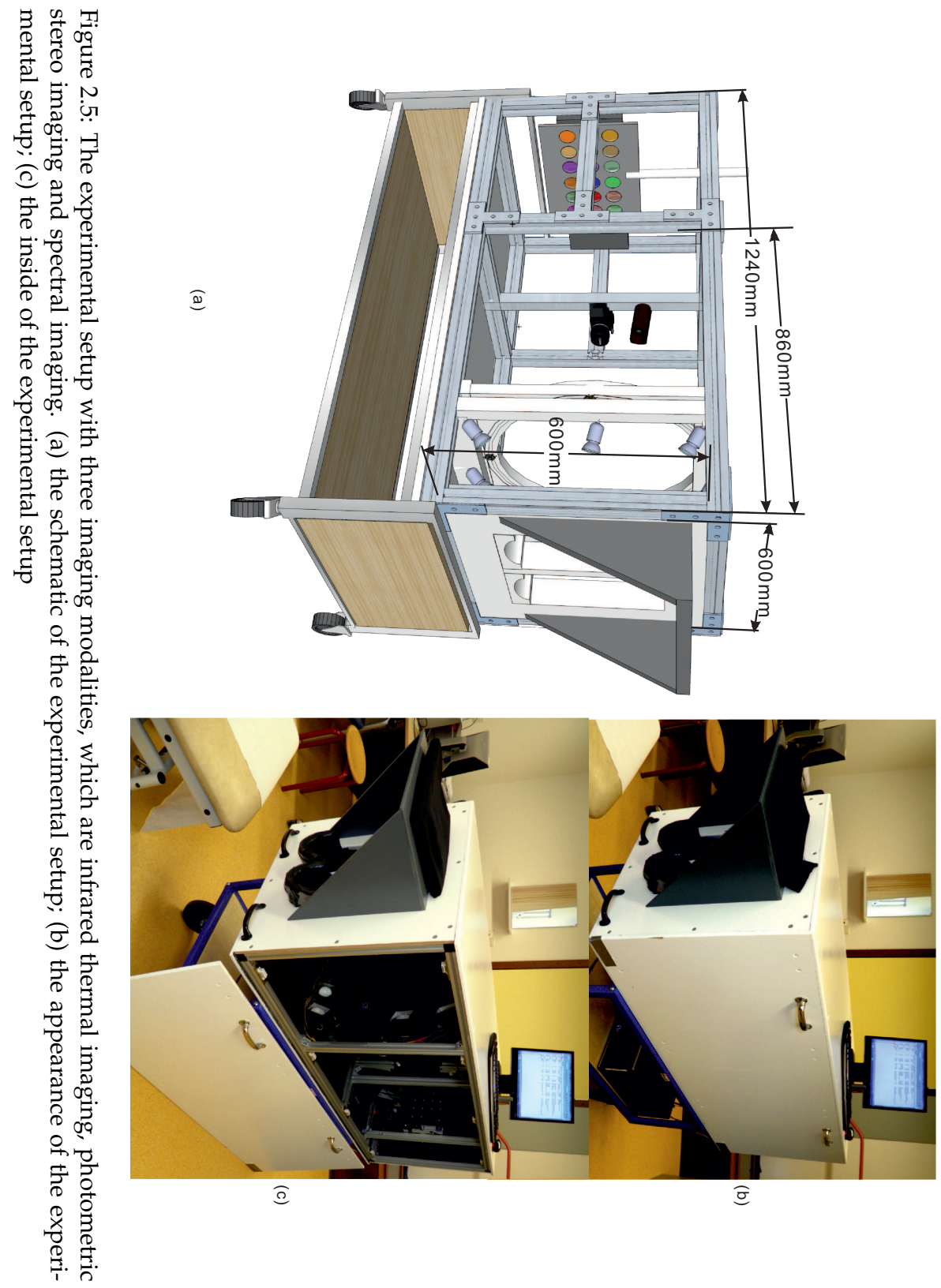



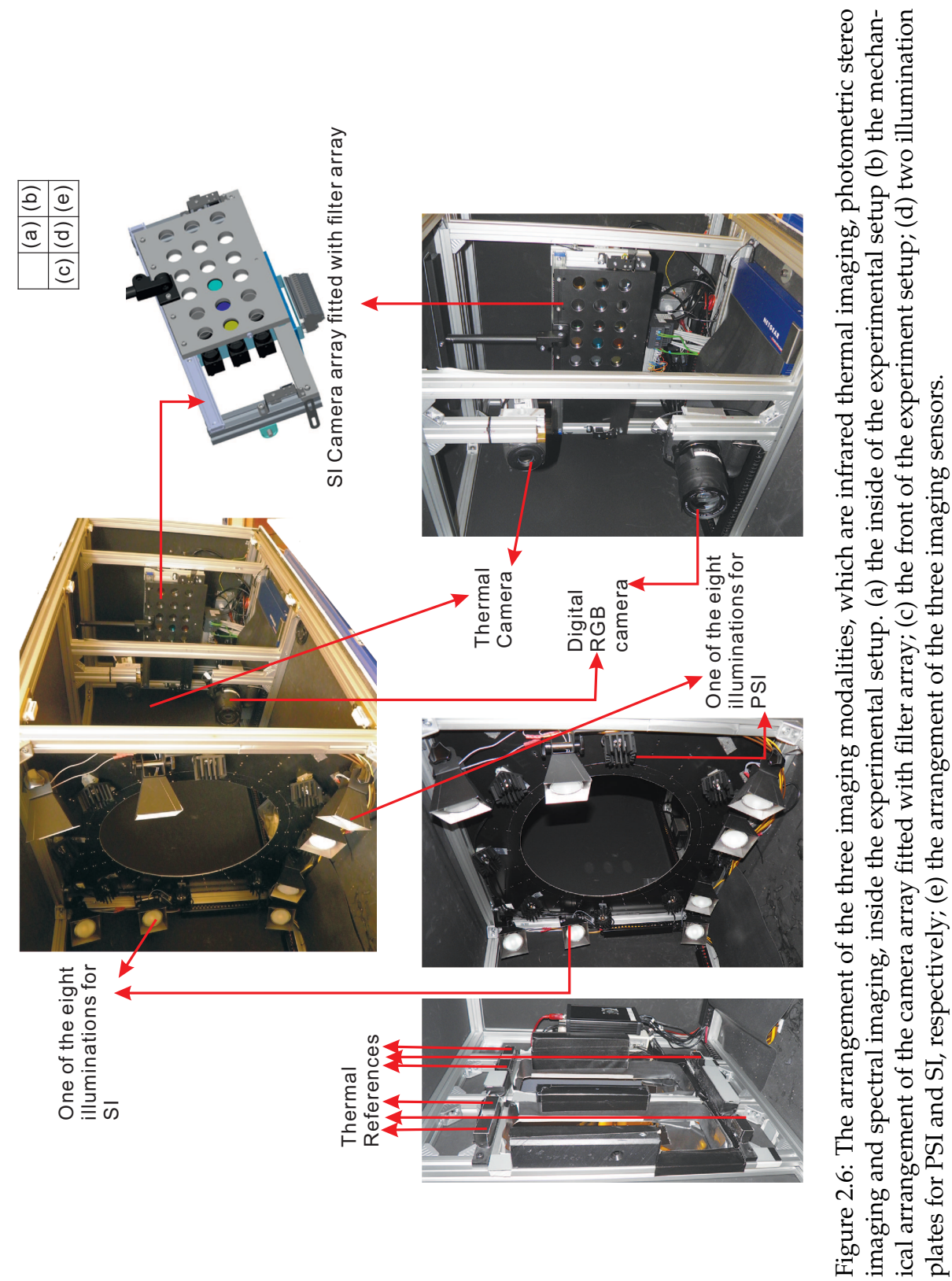


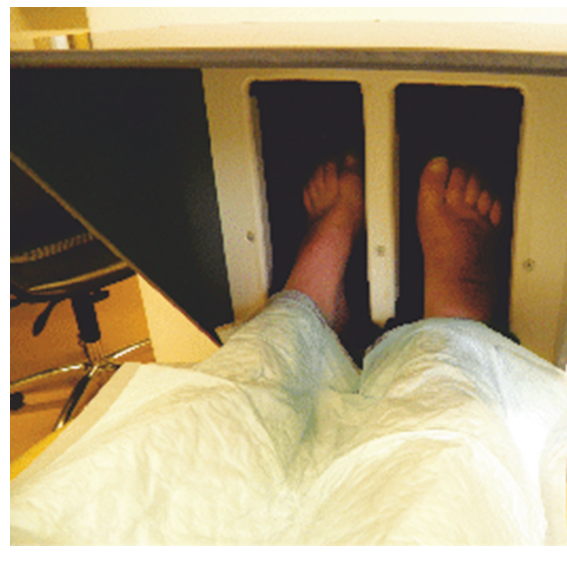

(a)

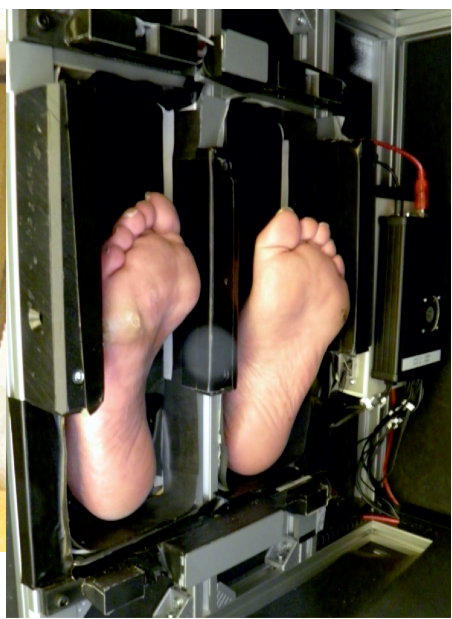

(b)

Figure 2.7: The positioning of the patient's feet for image acquisition. (a) the patient's feet were covered with a hospital cloth for for hygiene reasons. (b) the foot positioning inside the experimental setup.

measurement, they were all informed of the nature of the study. A written permission was given by the recruited patients to participate in this study. An example of the patient consistent form was given in Fig. A.1.

Before image acquisition, each foot was assessed by a clinical expert for the presence of diabetic foot complications, such as the presence of the ulceration, some important clinical pre-signs of ulceration (such as abundant callus, redness, fissures, blisters or other), or absence of any foot complications, on a simple live assessment form as shown in Fig. A.2. A foot ulcer was defined as a full-thickness lesion penetrating through skin dermis layer and into the subcutis. Abundant callus was defined as a callus formation requiring referral and treatment within three days. Each region of signs of foot complications was identified, reported and documented in the live assessment form. Patients were helped to place their foot on the foot support of the experimental setup. Measurements started after patients had rested for around 5-10 minutes on the treatment bench with barefoot. This was done to achieve equilibration of the foot temperature with the environment temperature. The feet were then placed on the foot support of the experimental setup. A hospital cloth was placed over the legs of the patients for hygiene reasons and one piece of black 
cloth was draped over the shield of the setup to block external ambient light entering the setup and to provide a homogeneous background.

The measurement was performed via a controlled software based on Matlab platform. It followed the sequence of TI, PSI and SI. During the process of TI, all illumination sources inside the experimental setup were turned off and turned back on during the acquisition of colour images with the digital camera for photometric stereo imaging.

During the PSI measurement, the eight illumination sources were turned on one by one. For each of the illuminators, one image was taken. As such, for each measurement, an image set of eight images was acquired by the same digital camera with 8 different illumination sources. The total measurement for PSI took around 90 seconds, during which the patients were requested to keep their foot as stable as possible to avoid any movement. The image acquisition for each set of the filters of SI imaging modalities could be finished within 1 second. For thermal imaging, the image acquisition time per patient was also within 1 second. Two digital colour images were taken at the beginning and the end of the measurement, respectively. The difference between the two digital images were illustrated on the screen to help the operator to check whether there was any foot movement involved during the image acquisition process. If so, a repeated measurement of the three modalities would follow.

A 30-minute interval was assured between measurements of different patients to eliminate heat residue from the illumination or from the body of the previous patient. 



\section{Statistical Analysis of Spectral Data: a Methodology for Designing an Intelligent Monitoring System for the Diabetic Foot}

\subsection{Abstract}

Hyperspectral imaging is a promising technique for detection and classification of (pre-)signs of ulceration on diabetic foot. However, the number of the spectral bands should be limited to avoid overfitting, which is critical for pixel classification with hyperspectral image data. The goal of this chapter was to design a detector/classifier based on spectral imaging with a small number of optical bandpass filters. The performance and stability of the design were also investigated.

The selection of a bandpass filter boils down to a feature selection problem. A dataset was built, containing reflectance spectra of 227 skin spots from 64 patients, measured with a spectrometer. Each skin spot was annotated manually by clinicians as 'healthy' or a specific (pre-)sign of ulceration. Statistical analysis on the data set showed the number of required filters is between 3 to 7 , depending on additional constraints on the filter set. The stability analysis revealed that shot noise was the most critical factor affecting the classification performance. It indicated that this impact could be avoided in future spectral imaging systems with a camera sensor whose saturation level is higher than $10^{6}$ per sensor element i.e. per pixel, or by post-image processing.

This chapter has been published in a slightly modified version as "C.Liu, J. J. van Netten, M.E.Klein, J. G. van Baal, S. A. Bus, and F. van der Heijden, Statistical analysis of spectral data: a methodology for designing an intelligent monitoring system for the diabetic foot, Journal of Biomedical optics, vol. 18, no. 12, p. 126004, 2013." 


\subsection{Introduction}

Diabetes Mellitus (DM) is one of the most common chronic diseases worldwide and continues to increase in population and significance [1,2]. Vascular and neurological disorders in lower extremities are common complications of DM. Approximately $15 \%$ to $25 \%$ of patients with DM eventually develop foot ulcers [3]. If not adequately treated, these ulcers may ultimately lead to lower extremity amputation[84]. The onset of diabetic foot ulcers is preventable by early detection and timely treatment of (pre-)signs of ulceration, such as callus formation, inflammation, and blisters. However, early detection depends on frequent assessment. This is not always possible, because self-examination is difficult or impossible due to the various health impairments caused by DM, and a sufficiently frequent examination by health care professionals would be too intrusive and too costly. Previous studies have shown the feasibility of such frequent assessment with help of telemedicine [32]. However, digital photography, as applied in those studies, misses the opportunity for automatic detection, thereby still requiring help from health care professionals. The ultimate objective of our project is to develop an intelligent telemedicine monitoring system that can be deployed for frequent examination of the patients' feet to timely detect (pre-)signs of ulceration. That is, we want to automatically detect (pre-)signs of ulceration in the diabetic foot, and discriminate these from healthy skin. No such system is currently available.

Spectral imaging (SI) is a promising modality to achieve this objective. SI is a hybrid modality for optical diagnosis, which obtains spectral data of the entire measured area and renders it in image form[43]. Medical hyperspectral imaging (MHSI) provides a non-contact and non-invasive diagnostic tool that is able to quantify relevant features such as tissue oxygenation and epidermal thickness [59]. Impairments in these features play an important role in the development of diabetic foot ulcerations and their subsequent failure to heal [48]. Based on these features, studies have been conducted with MHSI to assess the risk for ulcer formation [59], to monitor diabetic neuropathy [55], and to predict ulcer healing [57]. Besides these three options, an intelligent monitoring system should also be capable to automatically recognize ulcers and pre-signs of ulceration, such as abundant callus formation, inflammation and blisters, and discriminate these skin spots from healthy skin. This can be achieved with the help of statistical pattern classification techniques. SI has not yet been used for automatic recognition of (pre-)signs of ulceration in the diabetic foot with statistical pattern classification techniques. 


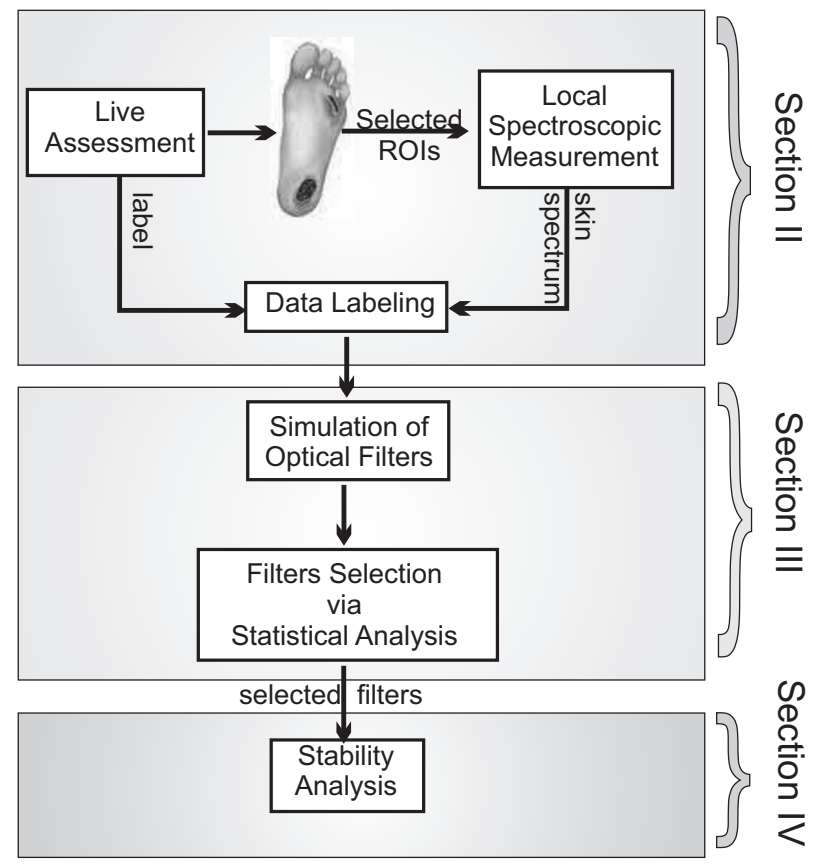

Figure 3.1: The schematic representation of the methodology in this study.

\subsubsection{Statement of the Problem}

The aim of the current paper is to describe the methodology for designing an intelligent monitoring system involving SI for the diabetic foot, with spectral data acquired from spectrometer measurements on skin spots. Such a design is hampered by the lack of a physical model signifying the difference between spectral data coming from skin spots that are healthy or (pre-)sign of ulceration (e.g. ulcers, abundant callus formation, inflammation, blister, etc.). The alternative is an approach that exploits the statistical properties of a training set of spectral data, which has been annotated by an expert clinician. This immediately confronts us with the problem arising from the high-dimensionality of the spectral data acquired by current available MHSI systems. Exploiting the statistical properties by training a pixel classifier using high-dimensional MHSI data tends to overfit [85], i.e. to adapt the classifier to accidental, statis- 
tically irrelevant peculiarities in the training set. This problem can be solved by a firm reduction of the dimensionality, by reducing the number of spectral bands (filters) employed in the SI system. Another advantage of such a reduction is that it will also increase the measurement and cost efficiency of the future system.

The methodology presented in this article will consist of determining suitable subsets of optical filters for the desired SI system by investigating their performance in discriminating different skin spots. Next, the stability of the selected filters in a simulated SI system will be analyzed with respect to shot noise and small changes of the experimental parameters.

\subsubsection{Outline of the Chapter}

Fig. 3.1 presents an overview of the followed methodology and an outline of the paper. In section II, the materials for collecting local skin spectral data on feet of diabetic patients with (pre-)signs of ulceration, the measurement procedure and resulting data set are described. In section III, the statistical analysis is performed on the acquired data, to search for suitable subsets of commercial interference bandpass filters. With the selected filters, the stability of the virtual SI system with respect to shot noise and small changes of the experimental parameters is investigated with a Monte Carlo method in section IV. Finally, the conclusions and future plans are presented in section V.

\subsection{Collection of Training Data}

\subsubsection{Data acquisition}

A spectroscopic system has been built to collect reflectance spectral data from the foot soles of patients with complications caused by diabetes. The experimental setup, as shown in Fig. 3.2, consists of a spectrometer (1), a broad-band illumination source (2), and a reflection fiber optics probe (3). The reflection probe (6.5 mm diameter) contains 7 fibers: 6 illumination fibers (Fiber 3a) surrounding 1 reader fiber (Fiber $3 b$ ). The probe is mounted in a probe holder made from black anodized aluminum, which is placed on the target skin area. It fixes the distance between the probe end and target skin area at $5 \mathrm{~mm}$. In this way, ambient light is blocked and a reproducible illumination under an angle of $45^{\circ}$ is achieved. The coaxial arrangement of illumination and recording fibers minimizes the influence of superficial scattering and specular reflection 
on the spectra. The recorded skin spectra cover the wavelength range from 360 to $1000 \mathrm{~nm}$ and contain 1127 channels with a bandwidth ranging from 0.6 to $0.53 \mathrm{~nm}$. The measurement area of the skin is around $60 \mathrm{~mm}^{2}$, which is suffi-

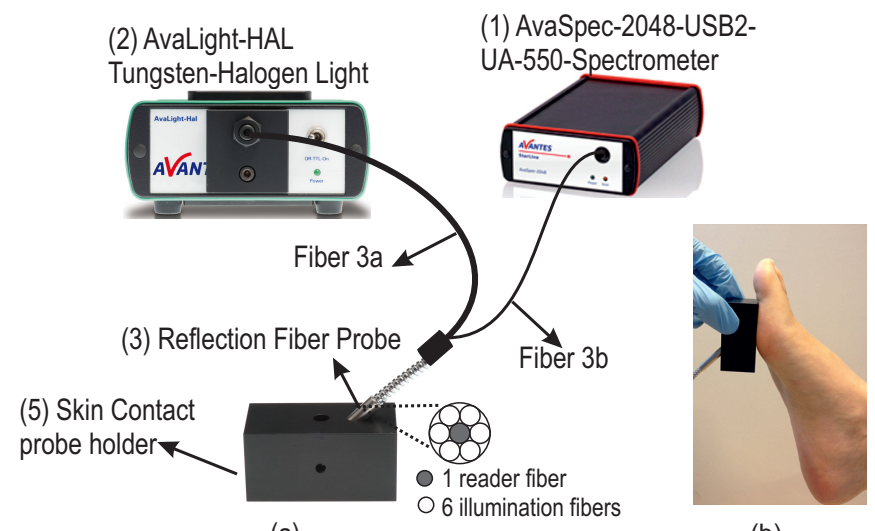

(a)

(b)

Figure 3.2: Schematic of the experimental setup for local spectral measurements;

cient for small (pre-)signs of ulcerations or a small sample of bigger (pre-)signs of ulcerations.

Prior to the acquisition of in vivo skin spectra data for each patient, the dark current, $D(\lambda)$, of the spectrometer is recorded when the light source is off. In addition, a spectrum $S_{\text {ref }}(\lambda)$ of a white spectral standard (WS-2, Avantes) is measured. This standard has a near-constant, high diffuse reflectance over the entire wavelength range, which is defined as $100 \%$.

The diffuse reflectance spectrum, $R(\lambda)$, of the measured skin ROI is then calibrated according to :

$$
R(\lambda)=\frac{S(\lambda)-D(\lambda)}{S_{r e f}(\lambda)-D(\lambda)} \times 100 \%
$$

where $S(\lambda)$ is the measured raw spectrum for the ROI.

\subsubsection{Patients Recruitment and Live Assessments}

Patients were recruited from the multidisciplinary diabetic foot clinic of the Hospital Group Twente, Almelo, the Netherlands. The patients included in this 
study were all diagnosed with diabetes and showed (pre-)signs of ulceration or had a history of ulceration. The average age of the 64 included patients was 65.7 years $(\mathrm{SD}=11.7$; range $35-95)$.

The reflectance spectra were collected from skin regions selected by experienced clinicians classified with the following live assessment scores:

1. ROIs on foot sole without (pre-)signs of ulceration. These were scored as 'Healthy';

2. ROIs on foot sole with ulceration. These were scored as 'Ulcer';

3. ROIs on foot sole with abundant callus. These were scored as 'Callus';

4. ROIs on foot sole with other less frequent pre-signs of ulceration, such as redness, fissure, blister and necrosis. These were scored as 'Rest'.

During the measurement, the probe holder was placed on the skin in two directions, which were along the width of the foot and the orthogonal direction. Each ROI was measured two times in each direction. In some cases, when the areas of the ROIs were smaller than $60 \mathrm{~mm}^{2}$ or on the edge of the foot, only two measurements along the first direction were performed. At least one 'Healthy' ROI was measured on each foot of a patient. The final spectra of each ROI was obtained by taking the average of all the four or two measurements.

\subsubsection{Summary of the Acquired Dataset}

Local reflectance measurements were performed on 227 ROIs from 64 patients, among which there were 95 'Healthy', 55 'Callus', 38 'Ulcers' and 39 'Rest'. The 'Rest' category bundled several very diverse skin conditions, each of which occurred only infrequently in this study (e.g. 6 necrosis, 5 redness, 3 blister, etc.). In addition, the corresponding areas of some of these ROIs were much smaller than the $60 \mathrm{~mm}^{2}$ measurement area, so that no pure spectra could be sampled from them. As a consequence, the within-class variation of the data in the 'Rest' category was very large. For this reason, the initial statistical analysis, presented in the next section, only focused on the data from the first three classes, which contained 188 of the measured 227 spectra. Examples of the measurements are given in Fig. 3.3. 


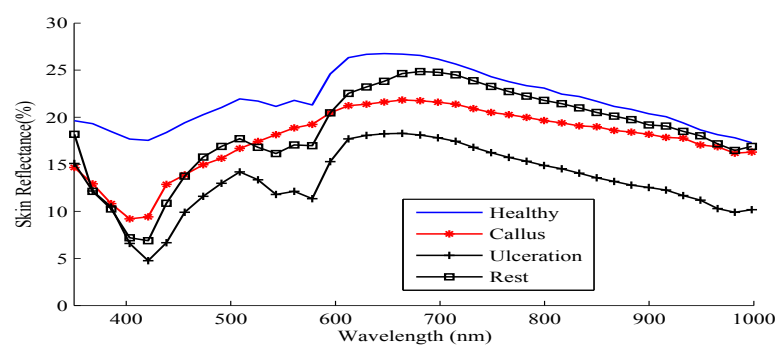

Figure 3.3: Examples of spectrum from different classes.

\subsection{Statistical Analysis for Selection of the Optical Filters}

In this section, the statistical analysis will be performed on the acquired skin spectra to search for the most informative optical filters for the future spectral imaging system.

\subsubsection{Methodology for Optical Filter Selection}

The functional structure of the statistical analysis is shown in Fig. 3.4. The database, consisting of the measured and manually classified skin spectra, is fed into a simulator of a large bank of optical filters. The characteristics of these virtual filters match the specifications of real, commercially available bandpass filters. The output data of all simulated filters, as well as the original measured data, is also applied directly to "Classification 1" and "Classification 2" to find the baseline performances, which can help "Filters Selection" to make decisions over the performance of the selected filters. The "Filters Selection" put all the outputs of the simulated filters in a pool to find the (sub-) optimal subset of filters for classifying the measured spectral data. In the subsequent process step "Classification 3", different classifiers are applied to find the most appropriate one that yields the best performance (i.e. accuracy of classification).

To make a comparison between the various selections of filters and classifiers, a relevant measure of the performance, such as the error rate (probability of misclassification), is essential. Since overfitting (resulting in a too optimistic estimate of the error rate) can easily occur if the same data is used both for training and for testing, $k$-fold cross validation and/or leave-one-out cross val- 


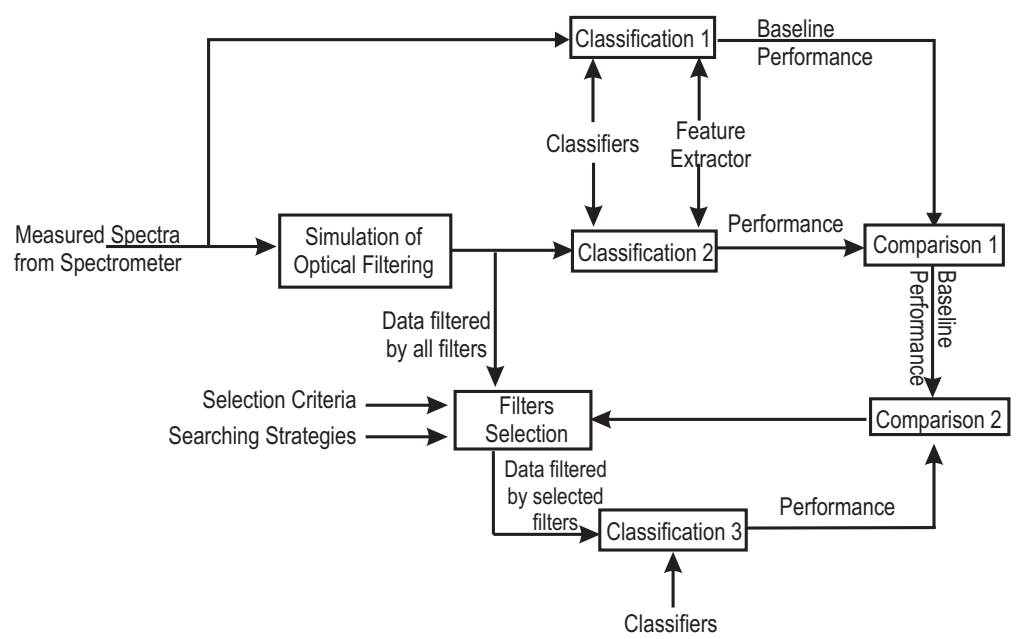

Figure 3.4: The functional structure of the data analysis that is applied to find the optimal subset of optical filters. All the data processing and pattern classification were performed on the Matlab R2012b platform with an additional toolbox PRtools V5 [heijden2004] in 64bits Windows 7.

idation [86] is applied to avoid that.

The design of a classifier, involves two aspects [86]: 1) selection of the type of classifier, and 2) the training of the parameters of the classifiers. The type of classifier (linear, quadratic, Parzen, etc.) defines the structure of the decision function that maps the features to assigned classes. The more complex the mapping is, the more parameters are needed for mapping, and the more the classifier is susceptible to overfitting. The complexity of a well designed classifier is a compromise between its ability to adapt its decision boundary to the requirements imposed by the statistical properties of the features at hand, and its ability to withstand overfitting.

There are two common approaches to reduce complexity, which are feature extraction (FE) and feature selection (FS). The FE approach extracts a set of new features using a simple function that maps the original $N$-dimensional feature space to a lower dimensional space. The features extracted do not necessarily have clear physical meanings. The FS approach only selects a subset of existing original features without any mapping. Thus, FS is the approach that is useful for finding the optimal subset of commercial available optical filters, as 
presented in Section 3.4.4, while FE will be used in Section 3.4.3 as a reference.

\subsubsection{Simulation of Optical Filtering}

In total, 148 interference bandpass filters were simulated, where the central wavelength $\lambda_{c}$ and the bandwidth $W_{d}$ of these filters were provided by real filters from two manufacturers: Edmund Optics [87] and Comar Optics [88]. The $\lambda_{c}{ }^{\prime}$ s changed from $400 \mathrm{~nm}$ to $1000 \mathrm{~nm}$ and the $W_{d}$ 's varied between $10 \mathrm{~nm}$ and $75 \mathrm{~nm}$. An overview of the parameters of all simulated filters is given in Fig. 3.5.

In addition to the parameters that are specified by the manufacturers, a third parameter $W_{t}$ was assumed to model the steepness of the filter. $W_{t}$ is the transition width between zero transmission and maximum transmission, which was set to $W_{t}=10 \mathrm{~nm}$. Fig. 3.6 shows the modelled shape of the transfer function $w(\lambda)$, where $W_{d}$ represents the full width at half maximum and the transition zone is modelled by half a cosine function.

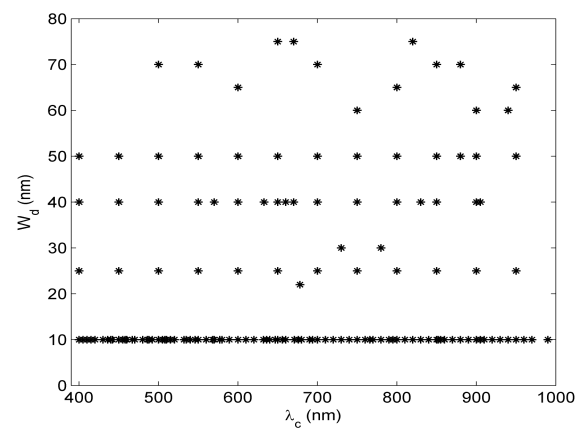

Figure 3.5: The parameters of the simulated filters. Each point represents a filter.

The $M_{S}=188$ spot spectra in the data set were denoted by $R_{m}(\lambda)$, where $m$ is the ROI number, $m=1, \ldots, M_{S}$. The $N=148$ filter transfers are denoted by $w_{n}(\lambda)$. The filter simulation is accomplished by a numerical approximation of:

$$
z_{n m}=\int w_{n}(\lambda) R_{m}(\lambda) d \lambda \quad\left\{\begin{array}{l}
m=1, \ldots, M_{S} \\
n=1, \ldots, N
\end{array}\right.
$$




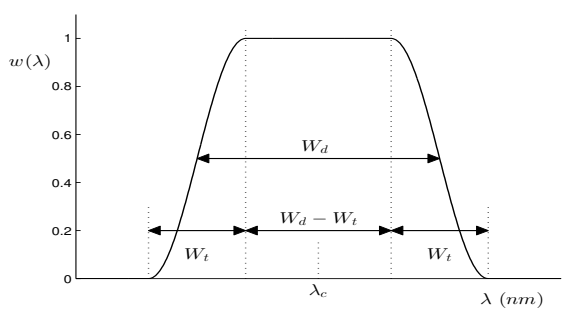

Figure 3.6: The modelled filter transfer and the definition of parameters.

The filter outputs $z_{n m}$ of each measured area are arranged in $M_{S} N$ dimensional vectors $\mathbf{z}_{m}$, called feature vectors. The true class for each ROI is available from the diagnosis by experienced clinicians. This yields a labelled training set of $M_{S}=188$ ROIs, $K$ classes ( $K=3$, 'Healthy', 'Ulcer' and 'Callus' ), and with each spot an associated $N$-dimensional feature vector $(N=148)$.

\subsubsection{The Baseline Performance}

In order to find a reference for the effects of filter selection, first the performance of classification was assessed with the original measured data and when all filters were used. Direct application of a linear classifier to the full feature vectors $(N=148)$ yielded an error rate as high as $70 \%$. Other classifiers yielded likewise performances. The explanation for this behaviour is overfitting. According to a simple rule of thumb [89], to avoid overfiting in a linear classifier, the number of samples should be at least about five times the number of features (= number of filters). The present case did not fulfil this, and the number of features should be reduced before applying classification. Linear FE was applied to accomplish that.

Linear FE is a linear mapping from $\mathbb{R}^{N}$ to $\mathbb{R}^{D}$ with $D<N$ implemented by a $D \times N$ matrix $\mathbf{W}$ :

$$
\mathbf{y}_{m}=\mathbf{W} \mathbf{z}_{m} \quad\left(m=1, \ldots, M_{S}\right)
$$

Two methods are commonly used: linear Principal Component Analysis (PCA) and Linear Discriminant Analysis (LDA). The goal of PCA is to find the $D$ dimensional linear subspace of $\mathbf{z}_{m}$ that preserves as much variance in the data as possible. Often, $D$ is chosen such that, for instance, $99 \%$ of the variance is 
still in the linear subspace. The PCA projection was always scaled by the mean class standard deviations in this article.

LDA is a feature reduction method that takes class information into account. If there are $K$ clusters in an $N$-dimensional space, the linear subspace that separates the clusters optimally is at most $D=K-1$ dimension. Hence, for a data set with $K$ classes, LDA seeks to determine the $K-1$ linear subspace to preserve as much class-discriminatory information as possible: it simultaneously tries to maximise the inter-class distance and to minimise the intra-class distance.

If the original feature space is high-dimensional, and the training set small (as in our case), LDA must be preceded with PCA to prevent numerical instability [86]. The dimensionality preserved in the preceding PCA should be optimized by cross validation. In our case, the optimal PCA subspace of the $148 \mathrm{D}$ space is a $28 \mathrm{D}$ space which is further reduced to a $2 \mathrm{D}$ space by LDA. The features extracted as such are illustrated in the scatter diagram in Fig. 3.7a. In Fig. 3.7b, the figure also shows the scattering of the two most important PCA features. These two features hold $98 \%$ of the variance.

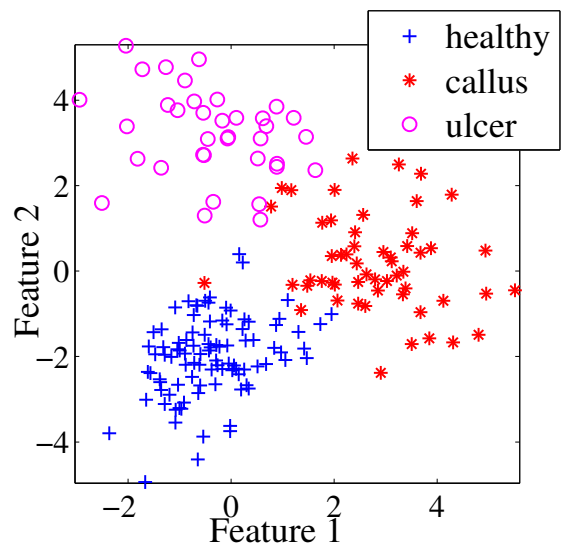

(a)

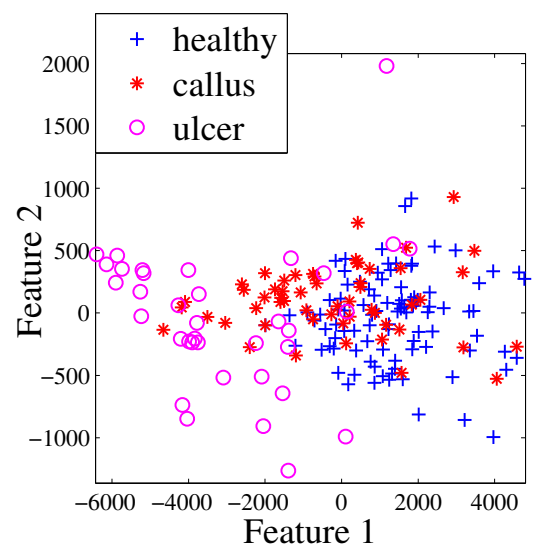

(b)

Figure 3.7: Scatter diagrams in two different feature spaces. 3.7a shows the feature space that is obtained by LDA (preceded by PCA). 3.7b shows the space of PCA features solely.

When PCA and/or LDA is applied to the filtered data, as in Eq. 3.3, each extracted feature is a linear combination of the original filter outputs. There- 
fore, an extracted feature can be regarded as the output of a single virtual filter. If the elements of $\mathbf{W}$ are denoted by $W_{d n}$, and the $d$-th element from $\mathbf{y}_{m}$ by $y_{d m}$, then Eq. 3.3 can be written as:

$$
y_{d m}=\int \sum_{n=1}^{N} W_{d n} w_{n}(\lambda) R_{m}(\lambda) d \lambda
$$

This effectively implements an optical filter with transfer function:

$$
w_{d}^{n e t}(\lambda)=\sum_{n=1}^{N} W_{d n} w_{n}(\lambda)
$$

The net optical filter transfers for the two LDA components are illustrated in Fig. 3.8.
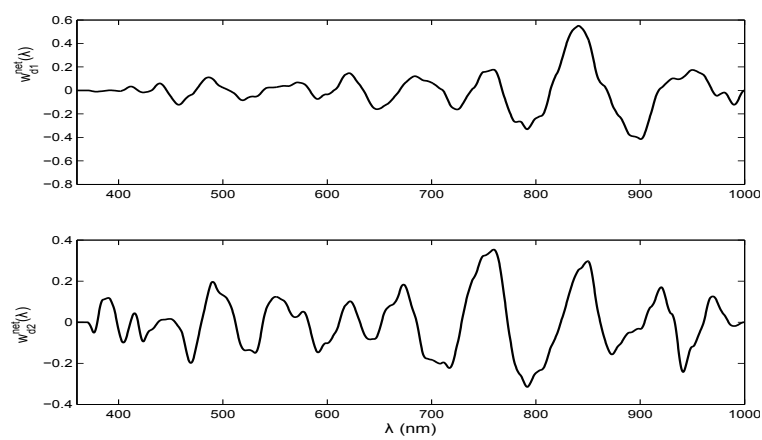

Figure 3.8: The net optical filter transfers for the two LDA components normalized to $[0,1]$

As shown in Fig. 3.7, the three classes are discriminated well by the two LDA features, indicating that a simple linear classifier suffices. We verified that by comparing its performance with the ones of a quadratic classifier, a Parzen classifier, a K-nearest neighbour classifier and support vector machine [86]. In all cases, the parameters were optimised using 10-fold cross validation. Besides, 10-fold cross validation was also used to assess the performance. Indeed none of them could out-perform the linear classifier, i.e. they all had similar performance, with a mean error rate of $9 \%$. Taking the time consumed for training and classification into consideration, the linear and quadratic classifiers were chosen for the filter selection. 
With LDA as the feature extraction and a linear classifier, the error rate of the full set of filtered data was $9 \% \pm 1 \%$ (obtained with 10 -fold cross validation). Exactly the same result was obtained with 28 PCA extracted features directly classified by the linear classifier, instead of indirectly via LDA. Following the same procedure to extract 2 features using LDA (preceded by PCA), the original measurement data (1127 spectral measurements per ROI) achieved an error rate that was almost the same. The averaged error rate differed less than $0.5 \%$. We conclude that the simulated filtering did not cause much information loss / gain, and an error rate of $9 \% \pm 1 \%$ would be used as the baseline, i.e the reference performance. For illustrative purpose, both of the error rates, from the original measurements (1127D space) and the data after filtering (148D space), will be presented in the following sections.

\subsubsection{Optical Filter Selection}

To select filters with FS techniques, i.e. to find a subset of features corresponding to the full set of filters, two choices have to be made: the search strategy to select candidate subsets, and the search criterion to evaluate these candidates.

Among the available searching strategies[90,91], the optimal search methods are not applicable, because their searching complexity grows combinatorially. The suboptimal strategies build up the subsets either incrementally (forward), or decrementally (backward). When searching for a relative small subset (less than 10 filters), backward selection is not adequate. Among the three forward strategies, which are sequential forward selection, plus-l-take-away- $r$ selection and sequential floating forward selection (SFFS), the most versatile approach, SFFS, was chosen[91].

The search criterion can be either a measure of the statistical distance of the class differences in the data, or it can be the estimated error rate of a classifier that is applied to the subset, the so-called wrapper. In this paper, one measure, the Mahalanobis distance, is studied, and two wrappers: the linear discriminant classifier (LDC) and the quadratic classifier (QDC). Both wrappers are evaluated with leave-one-out cross validation to avoid overfitting during searching.

To make a comparison between the various selections of filters and classifiers, a relevant measure of the performance, such as the error rate (probability of misclassification), is essential.

The main light-absorbing factors are melanin in the epidermis, and oxyhaemoglobin $\left(\mathrm{HbO}_{2}\right)$ and deoxy-haemoglobin $(\mathrm{Hb})$ in the dermis. The local concentration of each of these factors influences the measured skin spectrum 
in a specific way. In particular, $\mathrm{HbO}_{2}$ absorption features a strong maximum around 412nm and additional maxima at 542 and 577nm, while $\mathrm{Hb}$ exhibits peaks at 430 and 555nm[39]. Thus, these five wavelengths could provide more information about physical parameters, e.g. the epidermal thickness, and the oxygen saturation $[92,93]$.

The filter selection was carried out in two different ways: 1) all features / filters had the same eligibility, and 2) a constrained selection procedure was done in which center wavelengths, around the five wavelengths listed above, were evaluated and preselected with LDC. Once a subset of filters has been found, the subset must be evaluated empirically. This can be done with any type of classifier. Preliminary research showed that parametric-free classifiers, e.g. Parzen, did not perform better. Hence, we selected LDC and QDC once again for empirical evaluation. 10-fold cross validation was used to assess the error rate.

\section{Filter Selection without pre-selection}

The evaluation results are shown in Fig. 3.9. The SFFS strategy may return with a smaller number of features than the number that is requested when it detects that addition of features does not improve the search criterion. Six features were returned, when using LDC as searching criterion, while four features when using QDC as searching criterion.

When comparing the performances, the observations are:

a) With a set of appropriate chosen filters, the performance of all criteria compares well with the baseline performance.

b) The performance associated with the Mahalanobis search criterion converges slower than the other criteria. Apparently, the Mahalanobis criterion finds less strong features.

c) The Mahalanobis search criterion, evaluated with QDC, yields the lowest error rate at 5 filters.

d) The performances of LDC and QDC search criteria peak at 3 and 4 filters, respectively. Adding more filters does not improve the performance significantly, or could even worsen the performance.

e) For a smaller number of filters, the QDC classifier generally performs better indicating that the classes are better separated by quadratic decision functions than linear ones. 


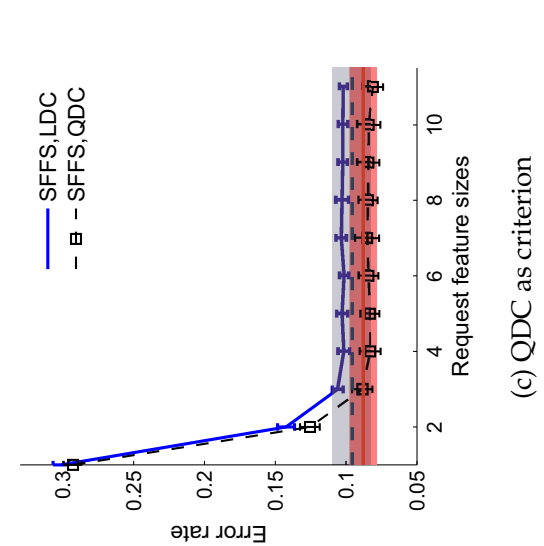

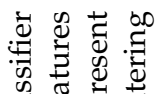

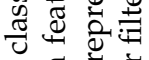
व 5 平焉 บे है वิ 을 픙 웡 छ छ के 䒕氙

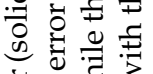
过 要运可 ป

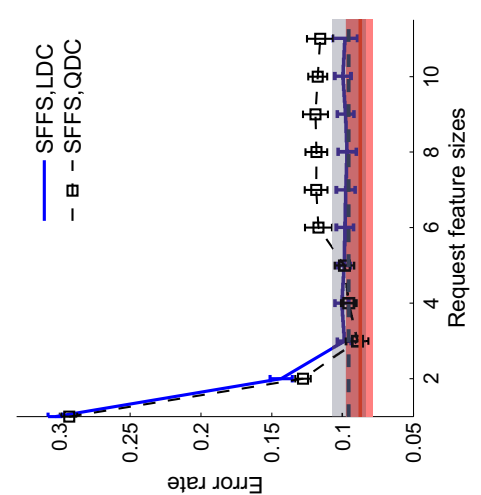
苛 U $\widetilde{\pi}$

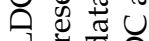

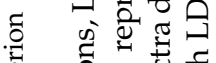

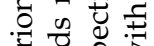
प्षे के 苞 ฮี 후음 巴ँ 艺忍导 하 क्षै

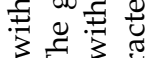
3 फ़ ऊ

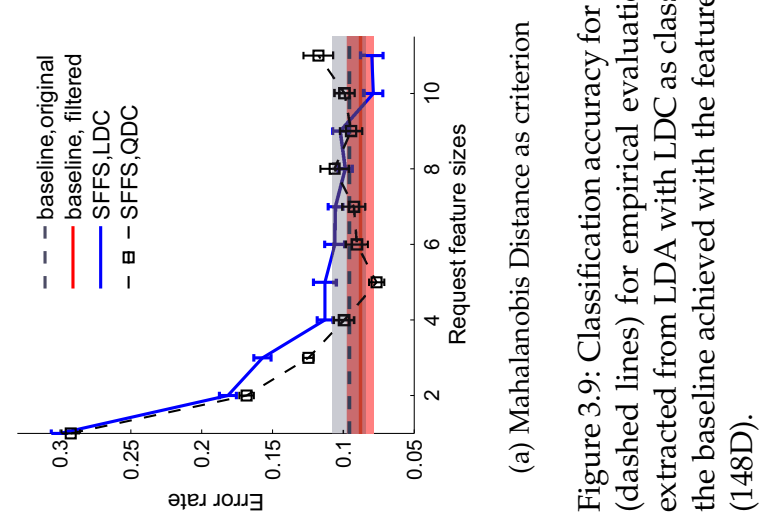


Table 3.1: The best subsets of filters (unconstrained search)

\begin{tabular}{|l|l|l|l|l|l|}
\hline $\begin{array}{l}\text { search crite- } \\
\text { rion }\end{array}$ & filter $_{1}$ & filter $_{2}$ & filter $_{3}$ & filter $_{4}$ & filter $_{5}$ \\
\hline Mahalanobis & $570(10)$ & $570(40)$ & $589(10)$ & $415(10)$ & $400(40)$ \\
LDC & $570(10)$ & $550(25)$ & $647(10)$ & & \\
QDC & $632.8(40)$ & $570(10)$ & $550(25)$ & $690(10)$ & \\
\hline
\end{tabular}

Each filter is defined by the parameter set " $\lambda_{c}\left(\mathrm{~W}_{d}\right)$ " given in $\mathrm{nm}$
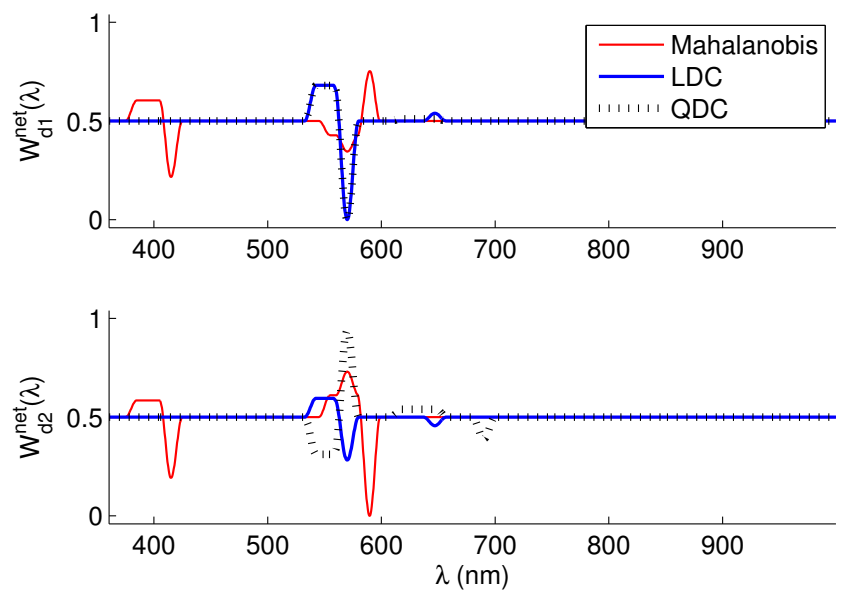

Figure 3.10: The net optical filter transfers that produces the two LDA components of the 3-class problem. These transfers, normalised to $[0,1]$, correspond to three feature subsets as given in TABLE 3.1.

The found subsets are given in TABLE 3.1. As can be seen in Fig. 3.10, each subset seeks information in the range of $550 \mathrm{~nm}$ to $600 \mathrm{~nm}$, and the LDA features are formed by differentiating filter outputs. This results in large impact on noise sensitivity, to be discussed in Section 3.5. The Mahalanobis distance seeks additional information in a range around $400 \mathrm{~nm}$. The LDC and the QDC both find information in the range around 650nm useful. 


\section{Filter Selection with pre-selection}

In addition to the five predefined filters, this constrained feature selection procedure selected up to 6 other filters from the rest pool. The results are shown in FIG.3.11. 10-fold cross validation was used to assess the error rate.

Again, the QDC as evaluation classifier often performed better than the LDC. The best results were obtained when QDC was used for the selection criterion as well as for empirical evaluation (Fig. 3.11c). With 10 features, the number of ulcer ROIs (i.e. 38) is somewhat less than 4 times the dimension, whereas it should be at least 5 times to prevent overfitting, according to the rule of thumb[89]. Apparently, it paid off to allow quadratic decision boundaries (rather than linear), although the additional parameters for the quadratic boundaries resulted in some performance loss due to overfitting.

The QDC in Fig. 3.11c obtains a minimum with 7 features. The performance improvement with the additional 3 features is not statistically significant. The filter parameters $\left(\lambda_{c}\right.$ and $W_{d}$, in $\left.\mathrm{nm}\right)$ that are associated with these features are, where the first five are the predefined filters:

$\begin{array}{llll}540(10) & 430(10) & 400(50) & 580(10) \\ 550(40) & 730(30) & 410(10) & \end{array}$

\section{The 'Rest' class}

Up to now, ROIs in the 'Rest' category were ignored. As said before, the 'Rest' consisted of a number of different subclasses that were often formed by tiny spots closely surrounded by healthy skin, callus, or ulcer. To see how the rest class behaves, Fig. 3.12 shows a scatter diagram of all spots in the posterior probability space that is spanned by $P($ callus $\mid \mathbf{y})$ and $P($ ulcer $\mid \mathbf{y})$. Here, y represented the 7D feature vector that was tabulated in Section 3.4.4.

The posterior probabilities were calculated under the assumption of class dependent, Gaussian likelihood functions, the base of the quadratic classifier [86]. The quadratic decision boundaries in the feature space are mapped to linear boundaries in the probability space.

When 'Callus' and 'Ulcer', with or without 'Rest', were conflated to one class 'Unhealthy', the estimated performances of the subsets selected are listed in TABLE 3.2. When 'Rest' included, the sensitivities dropped from $\sim 96 \%$ to $\sim 90 \%$ and AUC (Area under Receiver operating characteristic (ROC) curve) decreased with $0-0.2$, while the specificities all raised $\sim 1 \%$. Based on the table, all subsets selected with different criteria did similar and sufficient good work in classification. It is still hard to make a choice among these selected 


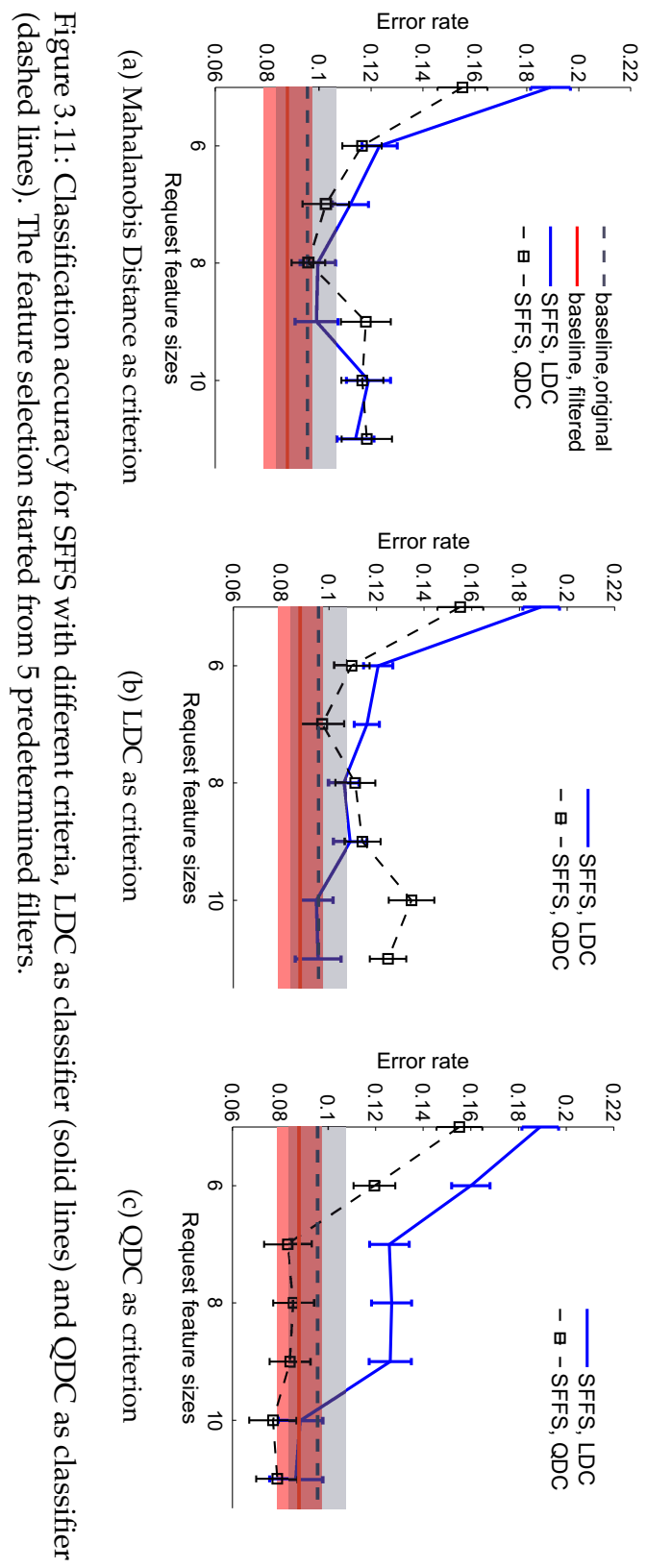


Table 3.2: Performance of the filters selected ${ }^{1}$

\begin{tabular}{|l|c|l|l|l|l|l|l|}
\hline Searching & \multirow{2}{*}{$\begin{array}{c}\text { Nr. of } \\
\text { criterion }\end{array}$} & \multicolumn{3}{|c|}{ No $^{\prime}$ Rest' $^{\prime}$ Class } & \multicolumn{3}{|c|}{ With 'Rest' Class } \\
\cline { 3 - 8 } & Sens $^{2}$ & Spec $^{3}$ & AUC & Sens & Spec & AUC \\
\hline Mahalanobis & 5 & $95 \%$ & $96 \%$ & 0.98 & $91 \%$ & $97 \%$ & 0.97 \\
& & & & & & & \\
\hline LDC & 3 & $95 \%$ & $92 \%$ & 0.97 & $93 \%$ & $93 \%$ & 0.97 \\
\hline QDC & 4 & $97 \%$ & $94 \%$ & 0.96 & $91 \%$ & $95 \%$ & 0.96 \\
\hline QDC $^{5}$ & 7 & $97 \%$ & $96 \%$ & 0.99 & $89 \%$ & $98 \%$ & 0.97 \\
\hline
\end{tabular}

${ }^{1}$ results get with 10 -fold cross validation

${ }^{2}$ Sens=Sensitivity; $\quad{ }^{3}$ Spec=Specificity; $\quad{ }^{5}$ With pre-selection;

${ }^{4} \mathrm{AUC}=$ Area under Receiver operating characteristic (ROC) curve;

subsets.

\subsubsection{Discussion of the Optical Filters Selection}

The 4 subsets of filters that are presented all operate in the wavelength range around $550 \mathrm{~nm}$. Two selections also operate in the vicinity of 400nm. These ranges are all in the biomedical window. None of the selections operate above $750 \mathrm{~nm}$. There is some resemblance between the filters that are based on the LDC and the QDC criteria, but apart from that, the different solutions do not have much intersection. The selection of filters is not very critical as different subsets perform equally well. In all subsets, the QDC outperforms the LDC. This suggests that the dispersion of the measurement data is class dependent. For class independent dispersion, the QDC degenerates into LDC. Since the dispersion of data is caused by the skin properties, and not by measurement noise, a class dependent dispersion makes sense.

The rest class is not included in the classifier design as yet. Fig. 3.12 shows that the 'Rest' ROIs are either situated at the three clusters that correspond with 'Healthy', 'Ulcer', and 'Callus', or situated in-between pairs of those clusters. The explanation is that the areas of 'Rest' ROIs are smaller than the measurement area. As a consequence, the measured spectra represent a mixed response from the 'Rest' ROIs and the surrounding areas belonging to one or several other classes. For instance, all 6 measurements for necrosis points in the 'Rest' category were classified as 'Ulcer', as shown in FIG.3.12. On the other hand, 3 out of 5 measurements from redness were classified as 'Healthy', 1 measurement was classified as 'Callus' and the other as 'Ulcer'. The same 'mixed area' 


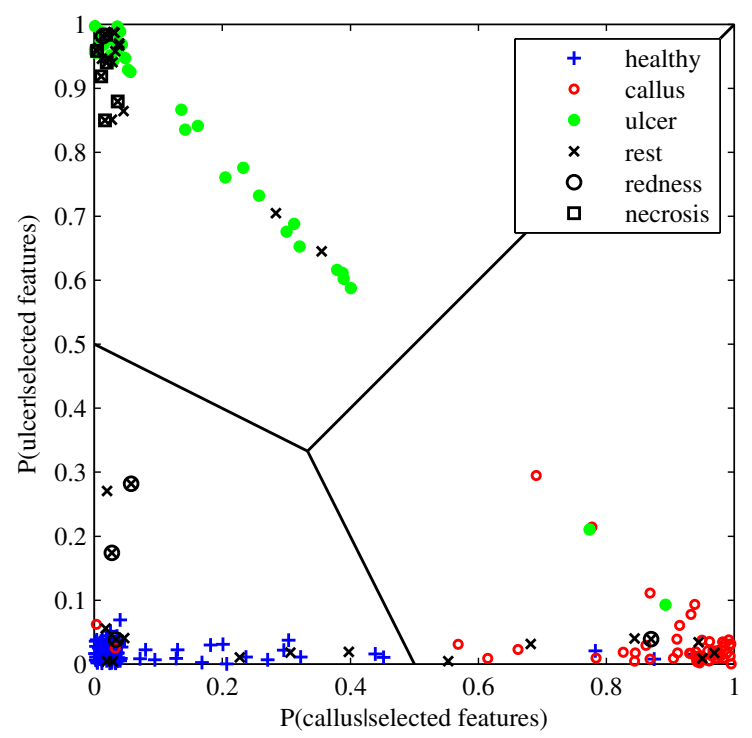

Figure 3.12: The scattering of the data set in the posterior probability space and the decision boundaries, under assumption of equal prior probabilities and equal cost of misclassification.

effect occurs in fact also for several ROIs labelled to the other classes. This also explains the phenomenon that the specificity rose when the 'Rest' class was added, as shown in TABLE 3.2.

For a real spectral camera, the pixel size will be much smaller than $60 \mathrm{~mm}^{2}$, the spectrometer measurement area, and the problem will not occur. However, the classifier needs retraining then, because the rest class is not included as yet.

\subsection{Stability Analysis}

In the previous section, the randomness of the data set mainly stemmed from the variations in physical properties of the skin. All other factors were idealised, with transfer functions that were assumably exactly known, and without possible shot noise. This section addresses the stability of the design, i.e. its 
ability to withstand small deviations of the transfer functions, and the ability to withstand noise.

\subsubsection{Monte Carlo Analysis}

The stability analysis was carried out by means of a Monte Carlo analysis applied to three different configurations of filter subsets and classifiers:

1. The subset of 4 filters that were selected without any constraints using SFFS/QDC as search strategy and with QDC as classifier (Fig. 3.9c).

2. The same subset as in 1), but with LDC as classifier.

3. The subset of 7 filters that were selected with SFFS/QDC, but with the first 5 filters preselected in the biomedical window, and with QDC as classifier (Fig. 3.11c).

Configuration 1) was chosen since its performance and its parsimony were the best that could be obtained. Configuration 2) was added to see whether the quadratic classifier was less stable than a linear classifier. Configuration 3) was chosen to see whether a large number of filters (a less parsimonious design) would degrade the stability.

The Monte Carlo stability analysis was done as follows. After random sampling of the parameter of a configuration, the error rate of that configuration was estimated using 10-fold cross validation. This was repeated 100 times, and the average over these 100 estimates are shown in Fig. 3.13. The procedure is repeated for four parameters: the center wavelengths $\lambda_{c}$, the bandwidths $W_{d}$, the widths $W_{t}$ of the transition band, and the maximum expected number of electrons. The latter parameter determines the SNR of the system.

The manufacturers specify tolerance regions for $\lambda_{c}$ 's and $W_{d}$ 's. For different $W_{d}$ 's and different coating approaches, the tolerances may be different. For example, when $W_{d}=50 \mathrm{~nm}$, the tolerance is $\pm 15 \mathrm{~nm}$ if traditional coated, and $\pm 5 \mathrm{~nm}$ if hard coated. When $W_{d}=10 \mathrm{~nm}$ the tolerance is $\pm 2 \mathrm{~nm}$. When $W_{d}=25$ or $30 \mathrm{~nm}$ the tolerance is $\pm 3 \mathrm{~nm}[87,88]$.

Note that the transmission coefficient of the spectral filters, which varies from $30 \%$ to $90 \%$ for the types of filters considered here[87, 88], need not be included in the stability analysis. This is because it can be assumed that the response of a given imaging system (including light source, image sensor, lens, and the filters) is calibrated for each filter using e.g. a white reference standard, and that it remains calibrated during the measurements. However, low transmission coefficients may influence the signal-to-noise ratio (SNR). 
SNR of the imaging system may be another important aspect that affects the classification stability. The random count of electrons, which are generated at the image sensor during the exposure time, defines the image output and decides the shot noise[94]. The probability of this count obeys Poisson distribution, and according to the property of which, the expectation of the count equals its variance, $E[$ count $]=\operatorname{Var}[$ count $]=\Phi$. Then, the SNR can be expressed as

$$
S N R=\frac{\Phi}{\sqrt{\Phi}}=\sqrt{\Phi}
$$

which grows with the expected number of electrons. So, $\Phi$ is another parameter of interest.

Each parameter was submitted to the Monte Carlo analysis by random sampling according to distributions of the parameters that modelled the tolerances:

- The central wavelengths were set to obey Gaussian distribution, with the nominal value as the mean, and a varying standard deviation $\sigma_{\lambda}$ during the experiment.

- The bandwidth was modelled by $(1+a) W_{d}$ in which $a$ had a Gaussian distribution with zero mean, and varying standard deviation $\sigma_{W_{d}}$ during the experiment.

- The variations of the transition bands were modelled by $(1-a) W_{t}$ where the distribution of $a$ was uniform between 0 and $T_{t}$. The parameter $T_{t}$ was the tolerance of the transition band.

- The shot noise was modelled by the parameter $\Phi$ which was the the expectation of the electron count that corresponds to the maximum output $y_{\max }$ (i.e. highest spectral reflectance value).

Then all features are transformed to the expected electron count using the factor $K=\Phi / y_{\max }$. According to Eq. 3.6, Gaussian noise was added to each feature with a variance that equals $\Phi$. Finally, the features were back-transformed by a factor $1 / K$.

Fig. 3.13 shows the results of the Monte Carlo analysis. The estimated errors all had standard deviations around $1 \%$.

\subsubsection{Discussion of Stability Analysis}

When comparing the results with respect to the three configurations of filter subsets and classifiers in Fig. 3.13, no substantial differences in behaviour can 


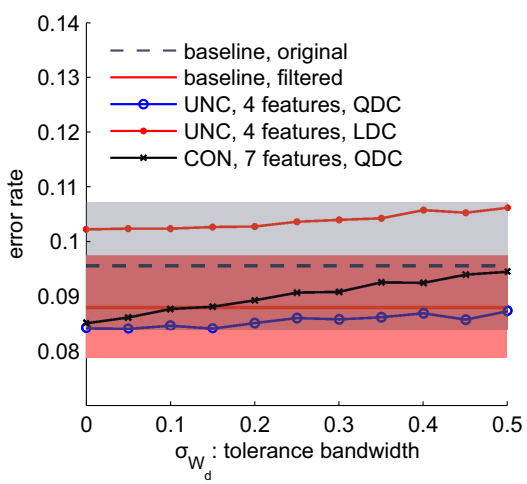

(a) Bandwidths of the filters

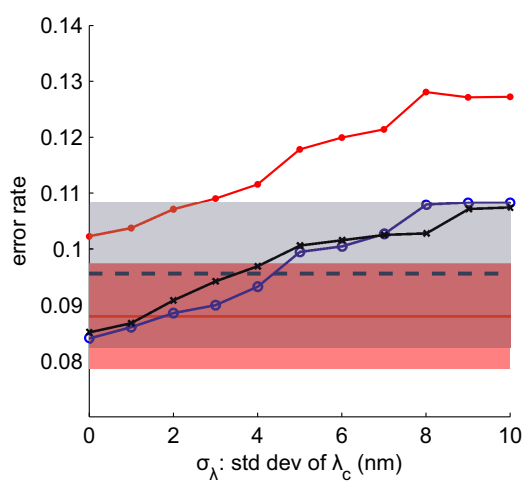

(c) Center wavelengths of the filters

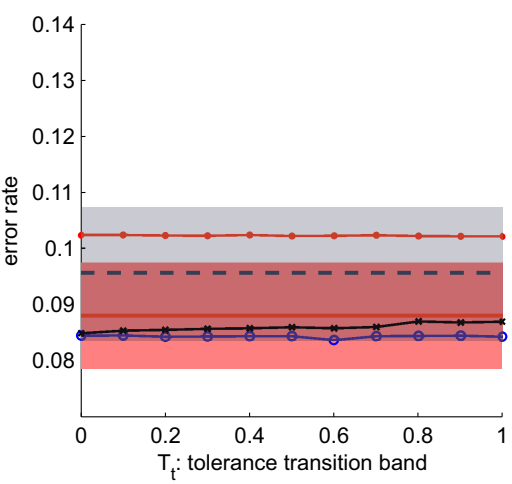

(b) Shapes of the filters.

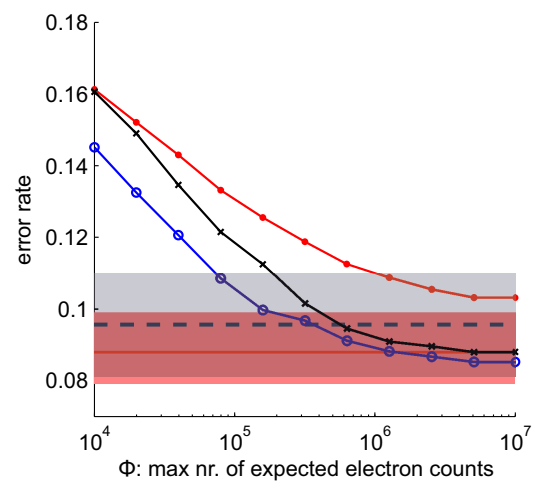

(d) Nr. of Electrons.

Figure 3.13: The influence of tolerances of filter parameters and the influence of shot noise. The performance is given in classification error rate. ' $\mathrm{UNC}^{\prime}$ and ' $\mathrm{CON}^{\prime}$ present unconstrained and constrained selection, respectively.

be observed. The linear classifier is not more stable than the quadratic classifier, and using only four filters instead of seven does not contribute much to stability either.

The shape of the filter transfer function is modelled by the bandwidth $W_{d}$ and by the width $W_{t}$ of the transition band. Fig. 3.13a and 3.13b show that the 
classification performance is not sensitive to changes of these parameters. The results suggest that such an assumption is not very critical: with extreme values of $W_{t}$ the modelled transfer functions vary between a rectangular window and a very smooth cosine window, but the performances are not affected.

Fig. 3.13c shows that shifts of the center wavelengths of the filters may reduce the performance. If an increase of error rate of $1 \%$ is permitted (about equal to the uncertainty of the estimated error rate), the standard deviation of the shifts should not be more than about $4 \mathrm{~nm}$. This requirement is equal to the specification of the low-cost commercial filters, so that it can be fulfilled easily when designing the spectral imaging system.

The most critical parameter appears to be the maximum expected electron count, $\Phi$. The explanation is that the differences between the outputs of filters are important for classifiers to discriminate different classes (as illustrated in Fig. 3.10). This may drastically reduce the SNR, i.e. even a small amount of noise has a large impact on the difference. Fig. 3.13d indicates that $\Phi$ should be above $10^{6}$ to avoid a performance decline due to quantum limitations. In a hyperspectral camera, such a high density of electrons can be realised by a) powerful illumination, b) large exposure time, c) large aperture of the optics, d) large pixel size, e) detective quantum efficiency close to $100 \%$, and f) transmission of the filters close to $100 \%$. However, the ultimate limitation is defined by the saturation level of the image sensor. For a high-quality industrial camera module with 4 megapixel resolution, a typical saturation level is $4 \times 10^{4}$. The figure shows that at that level the increase of error rate can be as high as $2 \%$; the subset of 7 filters a little higher than the one with 4 filters. If such a loss of performance cannot be tolerated, the effective electron count can be increased by using a sensor with large pixel size, and by summing over neighbouring pixels or subsequent frames. The price to pay for an increased pixel SNR is increased system costs, and reduction of spatial and temporal resolution.

\subsection{Conclusion}

The methodology for designing an intelligent monitoring system for the diabetic foot based on statistical pattern classification of spectral data has been proposed in this paper. To avoid the possible overfitting problem arising from high-dimensional spectral data and to design a system with a limited number of optical filters, a feature selection procedure was applied. This design methodology can also increase the measurement and cost-efficiency of the future system. The number of needed filters, in different subsets resulting from 
feature selection, ranged between 3 and 7 . The sets were all able to automatically discriminate between (pre-)signs of ulceration and healthy skin spots with a specificity of $96 \%$ and a sensitivity of $97 \%$. A stability analysis based on the Monte Carlo method revealed that the most critical design factor is the shot noise. The influence of the noise will be negligible if the saturation level of the image sensors is above about $10^{6}$ electrons. If this cannot be achieved, temporal or spatial averaging in the image should be applied, at the cost of the resolution.

One of the limitations of this study was the small training data set, in comparison to the abundant number of spectral bands. Such a set needs to be acquired during live assessment of patients using a spectrometer, and is expensive and therefore small. However, cross-validation during selection and evaluation helped generalising the analysis of the data set. The rest class could not be involved in the feature selection process, as this class was a repository of types of disorders that occur only infrequently. These spots were often so small that they fell below the resolution of the spot spectrometer. Ir is expected that in a real SI device with a higher resolution this problem will not occur. Also, sensitivity and specificity were acceptable with levels around $90 \%$ with the rest class included.

The first future step to be taken is to actually build the spectral imaging system with the optical filters as determined in this paper as an experimental setup first. Based on results obtained with such a setup, other relevant criteria concerning for example the ergonomics of the future system can be determined. These criteria will greatly influence the future system, and need to be investigated before issues such as hardware and costs can be extensively discussed.

Building an experimental setup containing the spectral imaging system with the optical filters described in this article will bring us one step closer to our ultimate goal: an intelligent telemedicine monitoring system that can be deployed for frequent examination of the patients feet to timely detect presigns of ulceration. Various options exist for application of such a system in daily clinical practice. For example, a system can be deployed at the home of high-risk patients who need close monitoring to prevent hospitalisation. Another option may be placement of the system with the general practitioner, to facilitate weekly or monthly screening of both high- and low-risk patients in an automated manner. Future clinical studies are needed to investigate the most cost-effective application of an intelligent telemedicine monitoring system for the diabetic foot in daily clinical practice. 



\section{A Front end for Automatic Detection of Diabetic Foot Complications using Spectral Images: a Statistical Approach to Pixel Based Segmentation}

\subsection{Abstract}

The objective of this Chapter is to develop pixel classifiers as front end for automatic detection of diabetic foot complications, taking annotations provided by clinical experts as ground truth. An experimental setup of spectral imaging system was built with the preselected optical bandpass filters. The procedure for this filter selection has been described in Chapter 3. Using the acquired spectral images from 63 patients, a dataset of pixels extracted from annotated regions were built and implemented for statistical analysis, and classification model development and validation.

Data from 42 patients were randomly selected as the training set. Performance evaluation with the rest 21 patients indicated that the front end achieved $78.8 \%$ sensitivity and $87.2 \%$ specificity in differentiating skin pixels between presence and absence of diabetic foot complications. Among the complications, $88 \%$ 'Callus' and $64 \%$ of 'Ulceration' were correctly detected. The output of these classifiers was sufficient as the input for back-end processing, in which higher level expert knowledge should be exploited to boost the performance to satisfactory levels in discriminating between different foot complications.

This chapter will be published in a slightly modified version as "C.Liu, J. J. van Netten, J. G. van Baal, S. A. Bus, and F. van der Heijden, A Front-end for Automatic Detection of Diabetic Foot Complications using Spectral Images: a Statistical Approach to Pixel Based Segmentation," 


\subsection{Introduction}

Diabetic foot complications, including ulceration and its pre-signs (e.g. inflammation, abundant callus and blisters), are major complications in patients with Diabetes Mellitus (DM). Ulceration provides an avenue for soft tissue infection, and may cause progressive tissue necrosis. If not adequately treated, the ulcers may lead to an increased risk of amputation and mortality [7]. Globally, diabetic foot ulcers result in one lower extremity amputation every 30 seconds, over 2500 limbs lost due to them per day [95]. Early risk identification, and subsequent treatment of diabetic foot complications in a timely manner, is demanded in amputation prevention and diabetes care. However, early onset detection strongly depends on frequent assessment. It is impractical and expensive to have frequent assessment by health care specialists. Examination by patients themselves has its limitations due to various health impairments caused by DM, such as limited joint mobility and reduced eyesight. Thus, any innovation for automatic assessment and detection of diabetic foot complications, which is noninvasive, non interactive and easy to use, may be beneficial for diabetic foot care. The ultimate objective of our project is to develop an intelligent telemedicine monitoring system for frequent foot assessment in order to detect diabetic foot complications in due course. Such a telemedicine system should be deployed at patients' homes or at health centres, making sure that patients obtain timely treatment and avoid subsequent amputation.

\subsubsection{Related work}

\section{Telemedicine system using digital photography}

Telemedicine systems based on digital photography have been built and tested for frequent foot risk assessment $[19,32]$. However, some diabetic foot complications are difficult to assess using digital photography [32]. Furthermore, such systems are not yet capable of automatic assessment of diabetic foot complications and inhibits their use as an intelligent telemedicine system. This limits their application and implementation.

\section{Skin Optics and Spectral Imaging}

The skin spectral properties are characterised by absorption and strong light scattering of incident light [46]. The absorption spectra of the main skin absorbers, Haemoglobin $\left(\mathrm{HbO}_{2}\right)$, deoxygen-Haemoglobin $(\mathrm{Hb})$ and melanin, are 
shown in Fig. 4.1. Different skin and sub-surface tissues have distinct or unique reflectance patterns. Small changes in skin surface and/or the subsurface tissue may result in obvious changes in skin spectral reflectance [46]. This motivated researchers to investigate the technologies for the skin spectral reflectance measurement to detect diabetic foot complications timely.

Spectral imaging (SI), a hybrid modality for optical diagnosis, has gained increasing attention in the investigation of spectral properties of foot skin from patients with DM. It measures the spectral data of the entire target area. The measurements are rendered into image form, providing a non-contact and noninvasive diagnosis tool to monitor diabetic neuropathy [55], to assess the risk for ulceration formation [59], and to predict ulcer healing [56,57]. Most of the pioneer studies followed the 'analytical approach' [96], which seeks to deconstruct the reflectance spectra into biologically and physically meaningful factors, such as a map of tissue oxygenation. These factors form a basis for diagnosis.

\section{Filter Selection for Spectral Imaging on Diabetic Foot}

Rather than finding out the biophysical causes behind the spectra exhibited by certain skin status, spectral data was examined to elucidate statistical metrics that correlate with different skin status in our previous study [40]. This was achieved with the help of statistical pattern classification techniques.

In this prior study, we exploited the statistical properties of the spectral data measured with a spectrometer to discriminate different skin spots (healthy or with diabetic foot complications) in patients with or at high-risk of diabetic foot complications [40]. Based on the statistical analysis, 7 spectral bands (filters) were selected for developing an SI system. The set of filters were able to detect the diabetic foot complications with $97 \%$ sensitivity and $96 \%$ specificity [40]. This firmly reduces the dimensionality of the SI data, and may increase the measurement and cost efficiency of the future SI system.

\subsubsection{Aim of this Study}

An experimental setup with a spectral imaging system was developed for patients' data collection, employing the preselected 7 optical bandpass filters. Statistical analysis on pixels from the acquired spectral images will be conducted, taking annotation provided by clinical experts as the ground truth. The objective of this study is to train and evaluate pixel classifiers for automatic detection of presence of the diabetic foot complications. 
At current stage, with limited number of patients and limited number of different diabetic foot complications available, this study was orientated as a study for proof of concept to distinguish between the skin with and without any diabetic foot complications, while leaving the study for differentiating the different diabetic foot complications to the future. These trained pixel classifiers in the current study can be used as the front-end processing of an automated system for detecting diabetic foot complications.

\subsubsection{Chapter Outline}

In Section 4.3, the SI system built for patient data collection, the measurement procedure and the patient recruitment are described. In Section 4.4, the image system geometrical calibration, data spectral calibration for reflectance map, the image preprocessing, and the statistical analysis are presented. Results and discussion follow in Section 4.5. Finally, conclusions are drawn in Section 4.6.

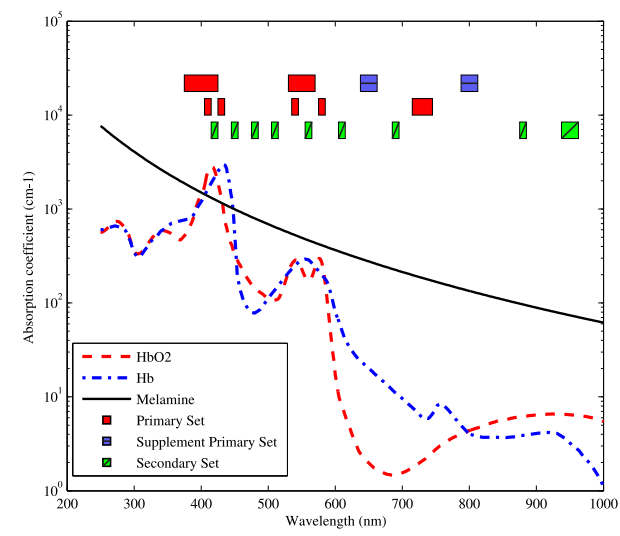

Figure 4.1: The absorption spectra for the main skin absorbers, $\mathrm{HbO}_{2}, \mathrm{Hb}$ and Melanin [39]. The rectangles in the plot corresponds to the distributions of the filters in two filter sets of our experimental setup. The red rectangle in the primary set are the optical filters selected [40]. The two blue supplement filters in the primary set, and the green ones in the secondary filter set were selected by visual inspection of the absorption spectra of $\mathrm{HbO}_{2}$ and $\mathrm{Hb}$. Selection of the spectral bands were done when there is large difference or small difference between these two spectra. 


\subsection{Materials and Image Acquisition}

An experimental setup, consisting of an array of nine cameras, eight illumination sources and two optical filter arrays (primary set and secondary set, each of which containing nine filters), has been developed for measurements on patients as shown in Fig. 4.2. Eight wide-band Quartz-Tungsten Halogen (QTH) lamps (Orsam GmbH, Munich, Germany, $400 \sim 1000 \mathrm{~nm}$ ) were mounted with equal distance to each other at the boarders of a square (Fig. 4.2a) at a distance of $465 \mathrm{~mm}$ from the target plane, which provided quasi-diffuse illumination with sufficient intensity at all wavelengths.

To eliminate the image acquisition time and avoid mechanical vibration involved when using filter wheel, we employed a camera array structure, arranged in a $3 \times 3$ matrix (Fig. $4.2 \mathrm{~b}$ ) consisting of 9 monochrome cameras (GigE Flea 3, Point Grey, Ludwigsburg, Germany) that can perform image acquisition almost simultaneously. The cameras were all fitted with Schneider Kreuznach Xenoplan 1.4/17 lenses (Bad Kreuznach, Germany), at a distance of $1240 \mathrm{~mm}$ from the target plane. The diaphragm of the lenses were set to F4.0 and fixed. Each camera covered a field of view $490 \times 370 \mathrm{~mm}^{2}$, with $1600 \times 1200$ pixels $(1$ pixel $\approx 0.3 \times 0.3 \mathrm{~mm}^{2}$ ). A movable filter plate with two sets of filters (Edmund Optics, York, UK and Comar Optics, Cambridgeshire, UK), arranged as two $3 \times 3$ array of filters, was mounted in front of the camera plate. The distribution of the spectral coverage of the filters is illustrated in Fig. 4.1. In addition to the 7 pre-selected filters, another 11 bandpass filters were also selected and integrated in the imaging system: 2 supplementary filters in the primary set and 9 filters as secondary set. The additional filters were selected based on visual inspection of the absorption spectra of the $\mathrm{HbO}_{2}$ and $\mathrm{Hb}$. This was done for a safe side in case the performance in detection of diabetic foot complications changed because illumination and detector in the imaging system were different from what was used in the spectrometer system for filter selection [40], which also gave us a second chance for filter selection for the final system.

Patients were recruited from the multidisciplinary diabetic foot clinic of Hospital Group Twente, Almelo, the Netherlands. The patients included in this study were all diagnosed with DM and showed diabetic foot complications, e.g. callus, blisters, redness, ulceration, or had a history of ulceration. From late January, 2014 to late May, 63 patients were recruited and measured, 52 males and 11 females. The average age was 65 years $(S D=9.3)$. Among them, there were 5 patients with Type I DM and 58 paints with Type II DM.

The measurements started after patients had rested with barefoot for around 5-10 minutes on the treatment bench. The patients were helped to place 


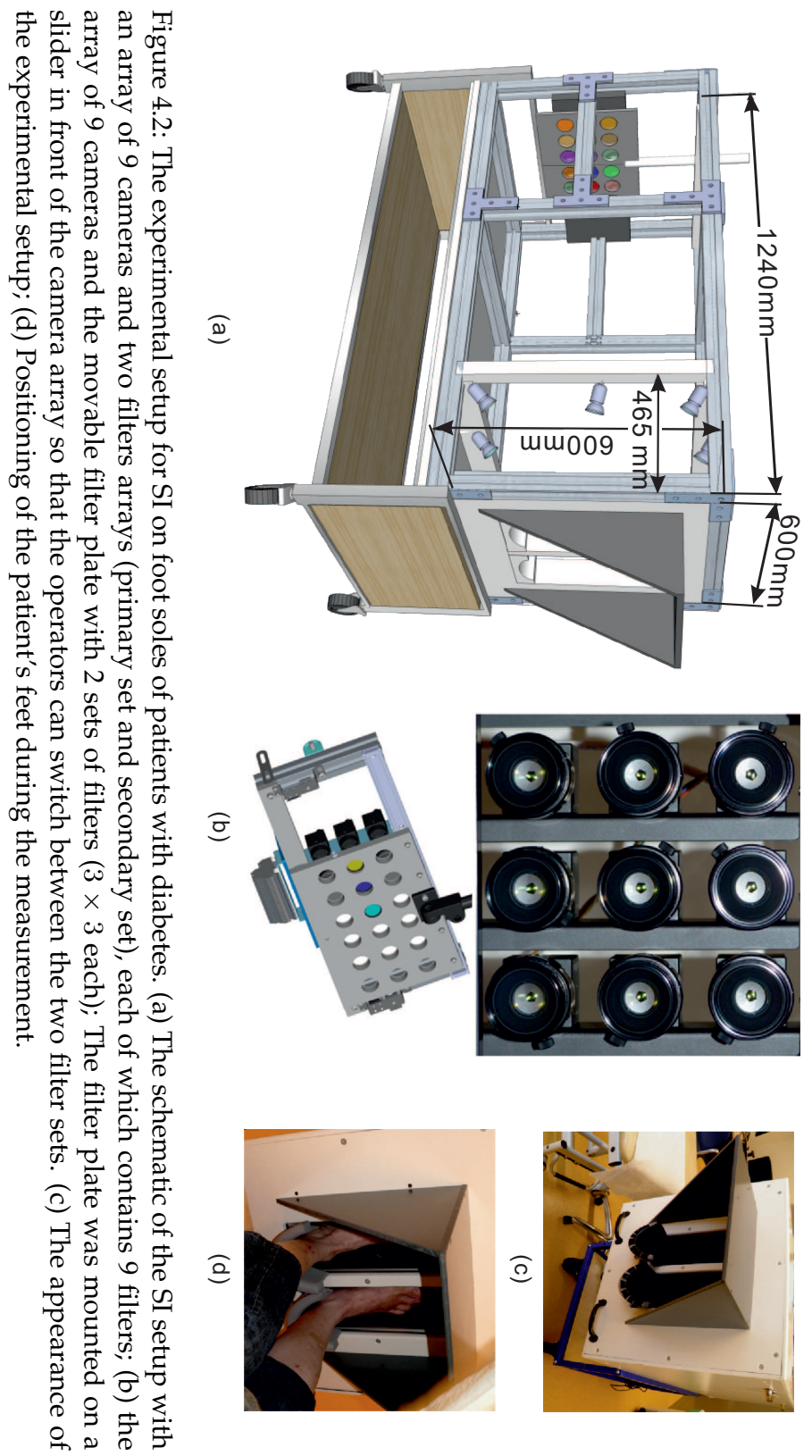


their foot on the foot supporters of the experimental setup (Fig. 4.2d). A hospital cloth was placed over the legs of the patients for hygiene reason. One piece of black cloth was draped over the shield of the setup to block external light entering the setup and to provide a homogeneous background. The image acquisition for each set of the filters was finished within 1 second. The total measurement time per patient for SI images acquisition with switching sets of filters is around 10 seconds.

For each patient, a live clinical assessment was performed by a wound care specialist, indicating all diabetic foot complications on the plantar surface of both feet on a paper form (as Fig. A.2). The assessment scores include 'Healthy' (absence of any foot complications), 'Ulceration' (the presence of ulceration), 'Callus' (an abundant callus formation requiring referral and treatment), 'Redness', 'Blister', 'Fissure' or 'Rest' (less frequent diabetic foot complications, such as necrosis). This live assessment was used as a reference for the validation of the pixel classification.

An example of the spectral image cube is given in Fig. 4.3.

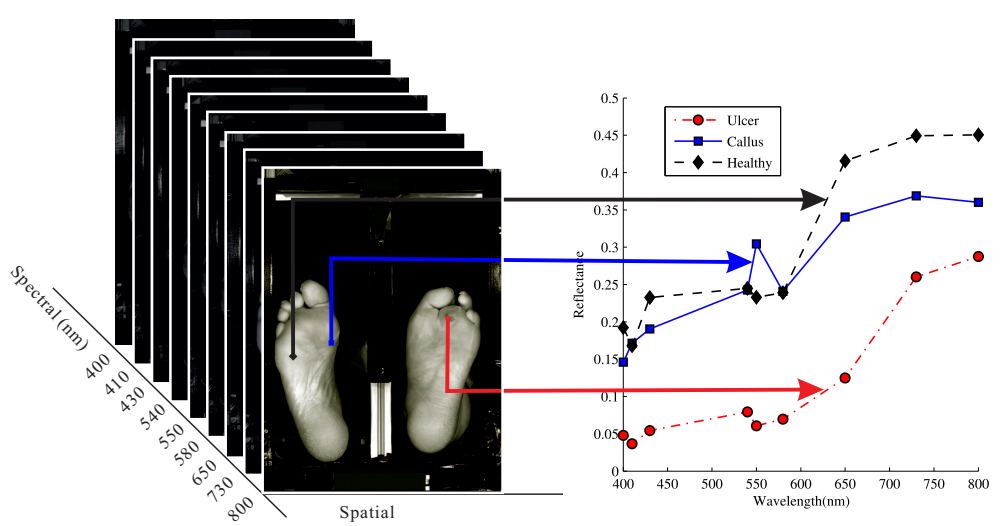

Figure 4.3: The spectral image cube with primary filters set and reflectance spectrum for three points selected. Indicated from the live assessment done by clinician experts, the score for the three points should be 'Ulcer' (Red Circle), ' Callus' (blue square) and 'Healthy' (black diamond) . 


\subsection{Spectral Image Processing and the Design of the Pixel Classifier}

The acquired spectral images were processed following the schematic functional diagram shown in Fig. 4.4. Before the imaging system was put into use for patients' data collection, the system had been calibrated with a planar chessboard target to register all cameras geometrically (Section 4.4.1). After converting the imaging measurement to spectral reflectance images (Section 4.4.2), the projective transformation parameters derived from the system geometrical calibration were utilised for aligning all the 18 images per patient. Each of the calibrated spectral image was normalised within the segmented foot area (Section 4.4.3) to eliminate the variation in the data set coming from the diversity among patients.

Based on the live assessment form filled by the wound care specialist before the imaging started, our clinical researcher carefully annotated every region of interest (ROI) on the aligned images (Section 4.4.5). These annotations could help to extract the pixel values within each ROI. Each pixel was then assigned with a predefined assessment score (class label). From all patients, the extracted pixel values with their labels were then merged as one dataset. Besides the spectral data, the vertical distance from each pixel on the plantar surface to the heel was extracted as an extra feature (Section 4.4.4). As such, each pixel acted as one object with 19 features in the dataset. Using this dataset, pixel classifiers were trained and evaluated for detection of foot complications (Section 4.4.6). Following the quantitative evaluation of the performance of the classifier, a preliminary evaluation in distinguishing different diabetic foot complications finalised the proposed front-end pixel classifier design (Section 4.4.7) to obtain an insight how the classifiers functioned in differentiating different foot complications.

\subsubsection{Spectral Imaging System Geometrical Calibration}

For the imaging system calibration, a target with chessboard pattern (each square with dimension $30 \times 30 \mathrm{~mm}^{2}$ ) was imaged with all cameras fitted with all filters covering foot region in the field of view.

The image registration was conducted with Scale Invariant Feature Transformation (SIFT) [97-99] for local feature detection and initial matching, combined with Random Sample Consensus (RANSAC) algorithm [100, 101] to eliminate the remaining mismatch points for robust estimation of the trans- 


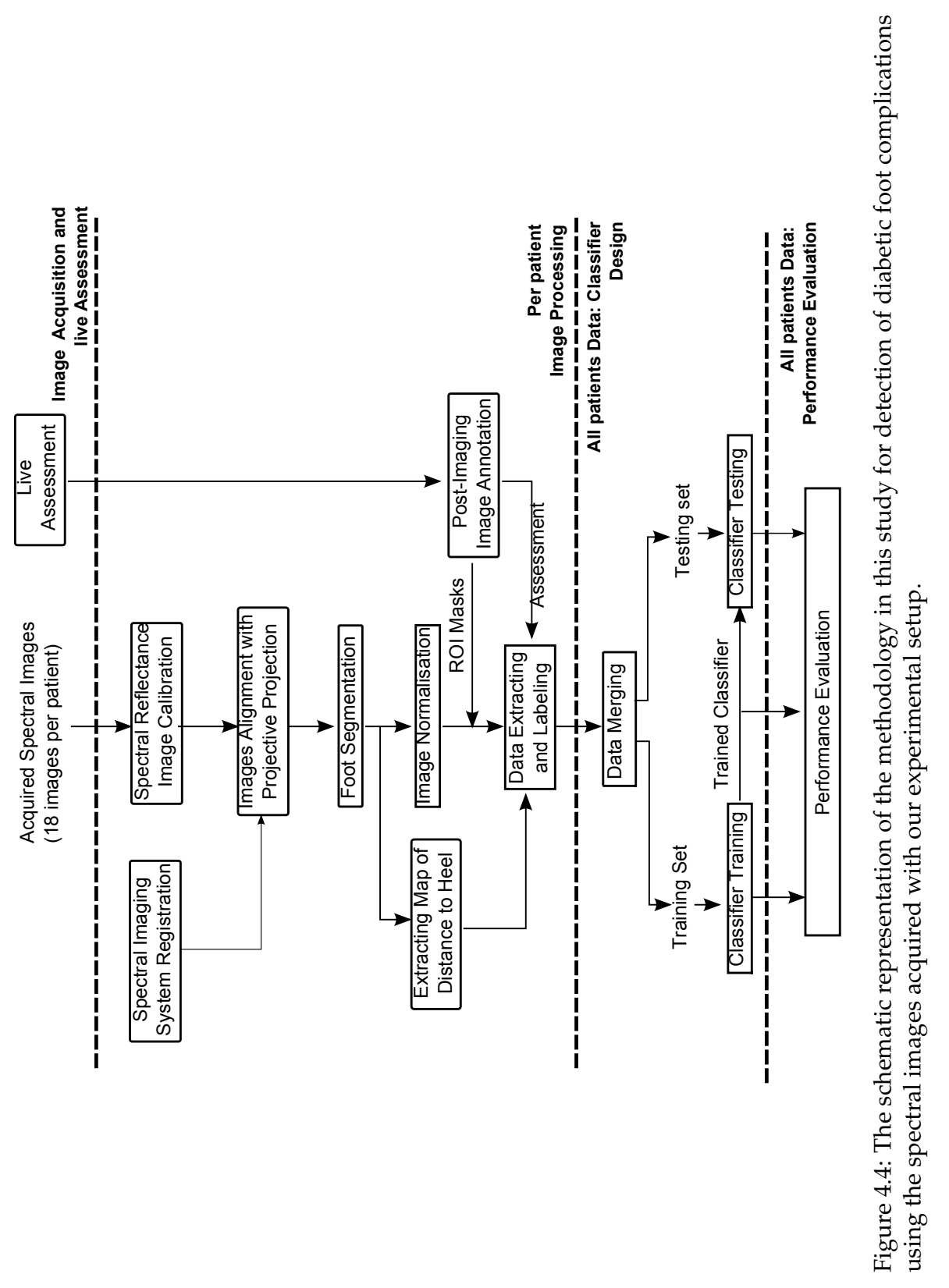


formation matrix.

SIFT is a popular technology in image stitching, matching, registration and $3 \mathrm{D}$ object recognition due to its ability to extract local features on images invariant of scale, orientation, illumination, image noise and small changes in viewpoints [97-100]. To extract these features, SIFT can be generally divided into 4 stages [99]:

\section{Scale-space extrema detection}

The first stage of computation searches over all scales and image locations to detect candidate keypoints. It is implemented efficiently by using a set of difference-of-Gaussian images covering a range of scales using a Gaussian pyramid to identify potential interest points that are invariant to scale and orientation by detecting the local minima and maxima though scale space.

\section{Keypoint localisation}

At this stage, the location and scale is determined for each candidate keypoint. Points with low contrast (sensitive to noise) and points that are localised at edges are deleted for their low stability.

\section{Orientation assignment}

At the third stage, each keypoint is assigned with an orientation based on local image gradient directions. This allows for the representation of each keypoint relative to this orientation, achieving invariance to image rotation.

\section{Keypoint descriptor}

The last stage of SIFT is to generate an array of SIFT descriptors, using the local neighbourhood around each keypoint. The location, scale, and orientation of each keypoint assigned with the previous steps are transformed are made identifiable by means of a descriptor vector that has tolerance to local shape distortion, changes in illumination or viewpoints.

With these descriptor vectors, initial keypoints from the reference image and keypoints from the target image were matched based on the Euclidean distance of these descriptor vectors. In our application for calibration of the spectral imaging system, we simply implemented the demo code for detecting and matching SIFT features provided in [102] on a Matlab 2013b platform. The nature of SIFT makes it a potential tool for (hyper-/multi-) spectral images registration. However, the problem of mismatching became more serious, for the number of incorrect matches increased when two images at two different wavelengths were matched, for example the matching between images at in- 
frared and Ultra Violet. This arose because imaging at different wavelengths gave significant differences in the acquired spectral images [103,104].

To deal with the remaining mismatching pairs of keypoints coming from the imprecision of SIFT, the RANSAC algorithm was implemented to make a more robust estimation of the transformation matrix. The RANSAC algorithm provides a general technique for calculating the model parameters (in our case, projective transformation parameters) mainly based on random voting principle in the presence of outliers in a reacted trial-and-error procedure. It can be summarised as follows:

- Inputs: The keypoints and their descriptors from both the reference and target images using the SIFT algorithm

- Output: The estimated projective transformation.

- The RANSAC algorithm:

1. Chose the transformation model to be estimated. In our case, the desired model was a projective transformation, i.e. homography.

2. Randomly select a minimum number of keypoints pairs ( $\mathbf{p}_{t}$ from the target image and $\mathbf{p}_{r}$ from the reference image) needed to estimate the transformation matrix $\mathbf{A}$ ( A should be a $3 \times 3$ matrix in homogenous coordinates: $\mathbf{p}_{r}=\mathbf{A} \mathbf{p}_{t}$ );

3. Apply the transformation $\mathbf{A}$ to the rest of the keypoints and count the inliers.

4. Calculate the symmetric transfer Error based on Euclidean distance $\left(D\left(\mathbf{x}, \mathbf{x}^{\prime}\right)\right)$ for each pair $E=D\left(\mathbf{p}_{r}, \mathbf{A} \mathbf{p}_{t}\right)^{2}+D\left(\mathbf{p}_{t}, \mathbf{A}^{-1} \mathbf{p}_{r}\right)^{2}$, if $E$ is smaller than a predefined threshold $\epsilon$, these points are inliners.

5. If the number of inliers exceeds the threshold, a fit is found and stop; Otherwise, repeat the computation steps 2-5.

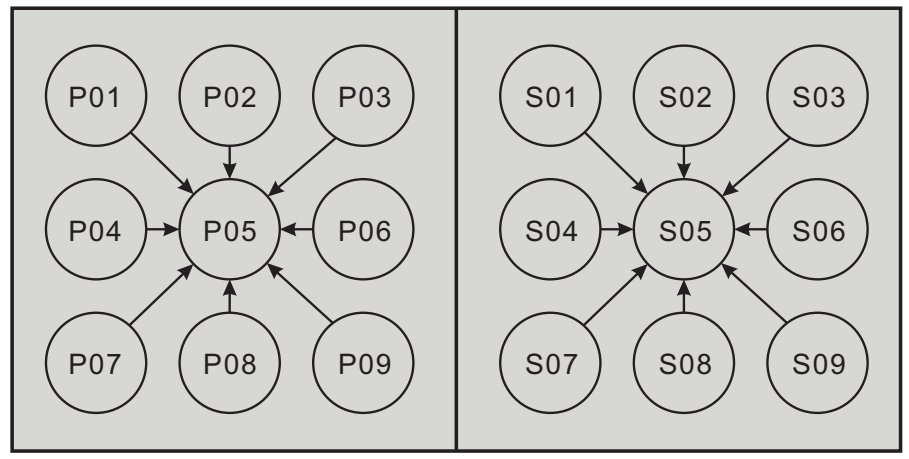

Figure 4.5: The arrangement of the filter plate and image registration order. ' $\mathrm{P}$ ' represents the primary filter set and ' $S$ ' for the secondary filter set. 


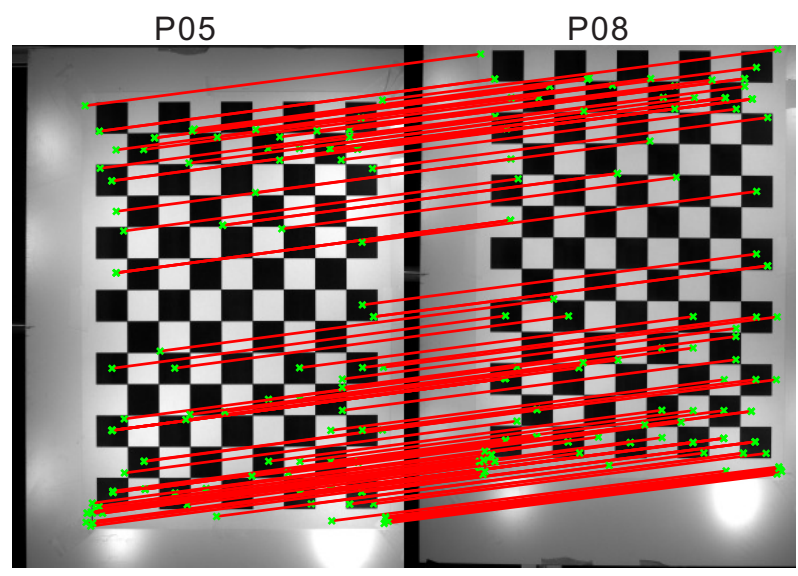

Figure 4.6: Example of images of the chessboard patterned reference target and key points pairs found with the SIFT and RANSAC algorithm. ' $\mathrm{P}$ ' represents primary set of filters. The indexing of the filters in the array can be found in Fig. 4.5.

In our implementation for estimation of the projective transformation, at least 4 matched pairs keypoints were needed to specify the homography. The filter array arrangement can be found as shown in Fig. 4.5. For either the primary filter set or the secondary filter set, we always take the images taken with the centre camera as the reference images and try to transform the other images to match with the centre ones. To eliminate the influence from the mechanical arrangement and slight changes in camera sensor response with different filters, we also registered the center image acquired when cameras were fitted with the secondary filter set (i.e. image taken with 'S05' in Fig. 4.5) to the center image taken when cameras were fitted with the primary filter set (i.e. image taken with 'P05' in Fig. 4.5). As such, all the images were registered with the image taken with the centre camera fitted with the filter 'P05'. One example of the image registration is illustrated in Fig. 4.6.

\subsubsection{Spectral Reflectance Image Calibration}

The reconstruction of the spectral reflectance images from measured raw Digital Numbers (DNs) or the photon count started from studying the camera sensor. Given an object with surface reflectance $R(\lambda, x, y)$, the measured DNs for this object, $Z_{j}(x, y)$ from the camera fitted with optical filter $j(j=1, \cdots, 18)$, 


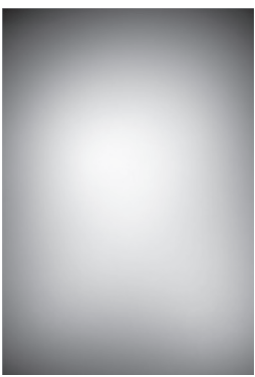

$550 \pm 40 / 2(\mathrm{~nm})$

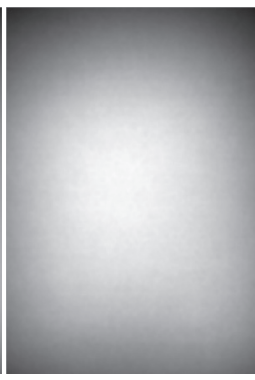

$410 \pm 10 / 2(\mathrm{~nm})$

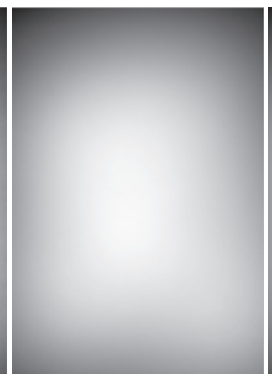

$730 \pm 30 / 2(\mathrm{~nm})$

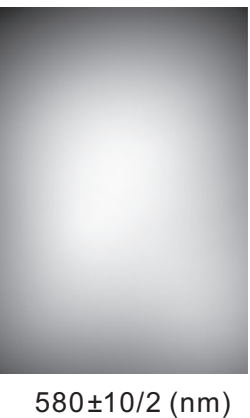

Figure 4.7: The acquired white reference with different cameras and optical filters. The central wavelengths \pm bandwidths $/ 2$ have been labeled.

whose Central Wavelength, $\mathrm{CW}=\lambda_{j}$ of which the bandwidth is $\mathrm{BW}=w_{j}$, can be written as [105]:

$$
Z_{j}(x, y)=\beta_{j} K_{j}\left[\int_{\lambda} L(\lambda, x, y) \tau_{j}(\lambda) R(\lambda, x, y) t_{j} d \lambda+d_{c}(x, y) t_{j}\right]
$$

where $\beta_{j}$ is the quantum efficiency and $K_{j}$ is the constant gain coefficient for the camera fitted with optical filter $j$, respectively. $L(\lambda, x, y)$ represents the at-sensor light irradiance, which was influenced by the uneven illumination, the geometrical effects of the lens (diaphragm, vignetting) and the geometrical mapping of the optical filters. $\tau_{j}(\lambda)$ denotes the optical transmission (including the filter transmission and the lens transmission), $d_{c}(x, y)$, the dark current and $t_{j}$ is the integration time.

Replacing the object surface reflectance $R(\lambda, x, y)$ with the reflectance of a white target $\left(R_{w}(\lambda) \approx C, C\right.$ is a constant), the measured white field DNs $W_{j}(\lambda, x, y)$ from the camera fitted with optical filter $j$ can be presented following Eq. 4.1. Examples of measured white references can be found in Fig. 4.7. The measured dark frame, taken when there was no light entering the camera sensor, can be represented as :

$$
D_{j}(x, y)=\beta_{j} K_{j} d_{c}(x, y) t_{j}
$$

Assuming the camera sensor model is linear, combining $Z_{j}(x, y), W_{j}(x, y)$, and $D_{j}(x, y)$, the reflectance of the object surface under calibration in the image of interest can be obtained as:

$$
R_{j}(x, y)=C \frac{Z_{j}(x, y)-D_{j}(x, y)}{W_{j}(x, y)-D_{j}(x, y)}
$$


where $R_{j}(x, y)$ is known up to a proportional constant since the reflectance of the white target, $C$, was unknown. The geometry of the white reference differs from the foot plantar surface, so this calibration would not be perfect.

Slight changes in the illumination provided by the QTH lamps can be expected due to ambient temperature, frequency of switching on/off, and especially the time delay between switching lamps on and actual image acquisition. In addition, the camera global gain may change over time. In order to correct the raw DNs for variations in overall the light intensity, the camera gain, and the filter transmission, two reference targets (plastic photographic grey card, $18 \%$ reflectance in visible range, Fotowand $\mathrm{GmbH}$, Sudwalde, Germany) were placed around the foot area but inside all cameras' fields of view. The grey cards were assumed to provide stable spectral reflectivity. The reflectance was measured with our spectrometer setup [40], as $r(\lambda)$. The calibrated reflectance $\left(R^{C}\right)$ can be adjusted as:

$$
\begin{aligned}
& R_{j}^{C}(x, y)=\frac{r\left(\lambda_{j}\right)}{\bar{R}_{j}^{\text {ref }}} R_{j}(x, y) \quad \text { with } \\
& \bar{R}_{j}^{\text {ref }}=\frac{C}{N} \sum_{n=1}^{N} \frac{Z_{j}\left(x_{n}, y_{n}\right)-D_{j}\left(x_{n}, y_{n}\right)}{W_{j}\left(x_{n}, y_{n}\right)-D_{j}\left(x_{n}, y_{n}\right)}
\end{aligned}
$$

where $\left(x_{n}, y_{n}\right)$ is the coordinates of the pixels of the references and $N$ is the number of these pixels.

\subsubsection{Foot Segmentation and Image Normalisation}

The Otsu's method is a histogram based global automatic thresholding method and is popular in image segmentation for it is simple and effective [106]. This also motivated our choice. The method was based on a simple idea that establishing an optimum threshold to minimise the weighted sum $\left(\sigma_{W}^{2}\right)$ of withinclass variances of the foreground (in our case, pixels on the surface of plantar foot) and background. Simultaneously, the between class variance, $\sigma_{B}^{2}$, is maximised.

The global optimal threshold can be estimated as:

$$
T_{o p t}=\underset{T}{\operatorname{argmax}}\left\{\frac{\sigma_{B}^{2}}{\sigma_{W}^{2}}\right\}=\underset{T}{\operatorname{argmax}}\left\{\frac{\omega_{f}(T) \omega_{b}(T)\left[m_{f}(T)-m_{b}(T)\right]^{2}}{\omega_{f}(T) \sigma_{f}^{2}(T)+\omega_{b}(T) \sigma_{b}^{2}(T)}\right\}
$$


where $m_{b}$ and $m_{f}$ represent the mean values of the background and foreground as a function of the thresholding level $T$, respectively; $\sigma_{b}$ and $\sigma_{f}$ denote the within class variance of background and foreground as function of $T$. $\omega_{f}(T)$ and $\omega_{b}(T)$ represent the cumulative probabilities of the two clusters.

Not images taken at all spectral bands were suitable for performing the Otsu' $s$ thresholding method to obtain the foot segmentation, because skin has different absorption of light at different wavelengths, filters have different transmission and cameras have different response, as such different intensities resulted. Empirically, the image taken with the optical filter, $730 \mathrm{~nm} \pm 30 / 2 \mathrm{~nm}$, was found suitable for the foot segmentation, due to the high contrast between the foot region and the background in the acquired image. An example of the foot segmentation was given in Fig. 4.8.

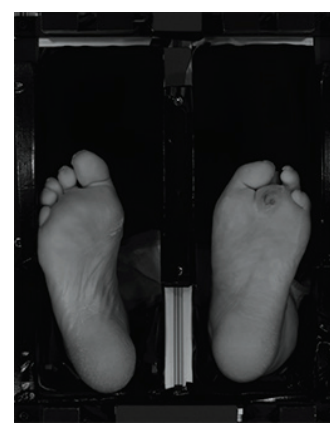

(a)

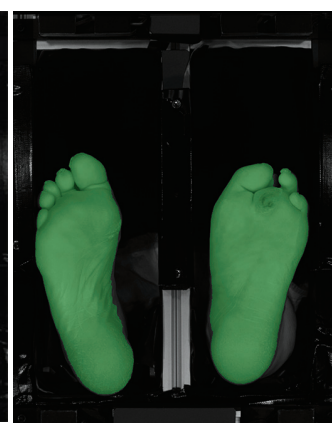

(b)

Figure 4.8: Image of patient foot taken with filters centered at 730nm (a) with the segmentation results (b). The green mask represents the segmented foot area.

To eliminate the within-class variation coming from the diversity among patients prior to the stage of designing classifiers, an image normalisation was necessary. For each pixel within the segmented foot region on every calibrated image per patient, the pixel values were normalised by assuming that the intensities in the foot area followed a normal distribution. The idea is simple to make the mean and the standard deviation to zero and one, respectively. The calculation was done according to:

$$
I_{j}(x, y)=\frac{R_{j}^{C}(x, y)-\mu_{j}}{\sigma_{j}}
$$

where $\mu_{j}$ represents the mean intensity of all the pixels within foot region on 
image $R_{j}^{C}$, while $\sigma_{j}$ is the standard deviation of the intensities.

\subsubsection{Extraction of the Distance Map to Heel}

Besides the measured spectral data, the location of specific diabetic foot complications is also important. For example, the skin that is thick on the heel due to pressure but that is not clinically classified as abundant callus, should be avoided to be classified as 'Callus' with our pixel classifiers. To issue this, we derive the distance map as an extra feature for each pixel on the foot plantar, as shown in Fig. 4.9.

To find the 'heel' point, the foot contour was rotated around the centroid point such that the midline stands vertical. The midline of the lower half of the foot was determined based on two steps: first, the midpoint of each horizontal segment that intersects the foot contours was taken; next, the least square linear fit was performed on these midpoints, and returned with the midline. When the foot contour stander vertically, the lowest point on the contour is defined as the heel point $\left(h_{x^{\prime}}, h_{y^{\prime}}\right)$. The vertical distance of the each pixel $\left(x^{\prime}, y^{\prime}\right)$ on the plantar surface to the heel can be easily extracted and normalised regarding the foot length, as:

$$
\begin{aligned}
D_{f}\left(x^{\prime}, y^{\prime}\right) & =\frac{h_{y^{\prime}}-y^{\prime}}{L_{f}} \times 100 ; \\
{\left[x^{\prime}, y^{\prime}\right]^{T} } & =\mathbf{T}[x, y]^{T}
\end{aligned}
$$

where $\mathbf{T}$ is the transformation matrix for the foot contour rotation and $L_{f}$ represents the longitudinal length of foot (along the $y^{\prime}$ axis) when the foot contour was rotated to stand vertically.

\subsubsection{Annotation and Dataset Construction}

Based on the live assessment form completed before imaging by the wound care consultant, our clinical researcher annotated the foot regions on the registered spectral images presented in pseudo RGB color. The three color channels in the pseudo RGB correspond to images taken with filter centred at $650 \mathrm{~nm}(\mathrm{R})$, $550 \mathrm{~nm}(\mathrm{G})$ and 430nm (B), respectively. Examples of the annotations can be found in Fig. 4.10.

The ROIs were annotated in a way to make sure that the risk of 'mixing effect' $^{\prime}$ was minimised, i.e. mixing different diabetic foot complications as one annotation, or mixing absence and presence of foot complications in one selected region. As the ulcers were usually surrounded by dead tissues and abundant 


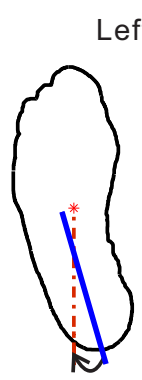

(a)

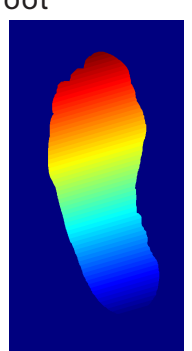

(b)

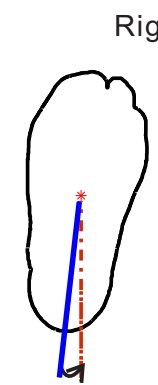

(c)
Right Foot

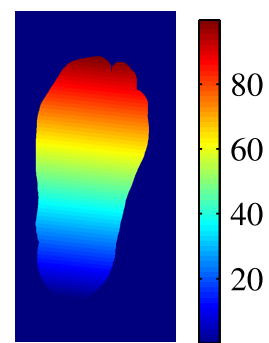

(d)

Figure 4.9: Distance map extraction for pixels with segmented foot plantar. (a) and (c) The medlines of the lower halves of the foot regions were used to align the foot vertically. (b) and (d) The extracted distance map for left and right foot, with a common colorbar indicates the normalised distance. The star indicates the centroid point of the foot contour. The blue solid line represents the midline of the foot.

callus, the selected region labeled with 'Ulceration' were generally smaller than the others to avoid such 'mixing'. Some less frequent diabetic foot complications, such as 'Blister', 'Fissure' ,'Redness', and 'Rest' were difficult to annotate in images presented with the (pseudo) RGB colors [32] and the area of the foot skin with such diabetic foot complications were quite small $\left(\approx 2 \times 2 \mathrm{~mm}^{2}\right)$. As a result, the annotated regions for these less frequent diabetic foot complications were as small as 11 pixels. On the other hand, skin with no diabetic foot complications was easily annotated with certainty. As such, regions annotated with 'Healthy' were much larger than the other regions.

Due to the illumination distribution changes on the field of view, the cast shadows formation resulting from the curved foot plantar surface, and the skin folds from the foot poses, the within-class variation of 'Healthy' skin in the acquired images was very large. To issue this problem, we added three sub categories (classes) to the category (class) without complications, which were

- 'Healthy Shadow': skin with no diabetic foot complications but with presence of cast shadows. The 'Healthy Shadow' regions usually were found at the root of the toes.

- 'Healthy Border': skin with no diabetic foot complications located at the border of foot. Due to the illumination changes, the pixels at the border of the foot always have lower intensities than the other pixels on the plantar foot. 
- 'Healthy Wrinkle': skin with no diabetic foot complications but with skin folds.

As such, the total data were clustered into 10 classes, 'Healthy' and its three subclasses mentioned above, 'Ulceration', ' Callus' , 'Blister', 'Fissure' , 'Redness' and 'Rest'. Each pixel within the annotated region was treated as one object in the dataset, with 19 features (the spectral image taken with 18 optical filters and the extracted distance map to the heel).

Worth to mention that the distance extracted for 'Healthy' category were adjusted to make it follow a uniform distribution within $[0,100]$. This was done because the 'Healthy' can locate at any position on distance map of the plantar foot and we do not want to affect the statistical properties of 'Healthy' distance

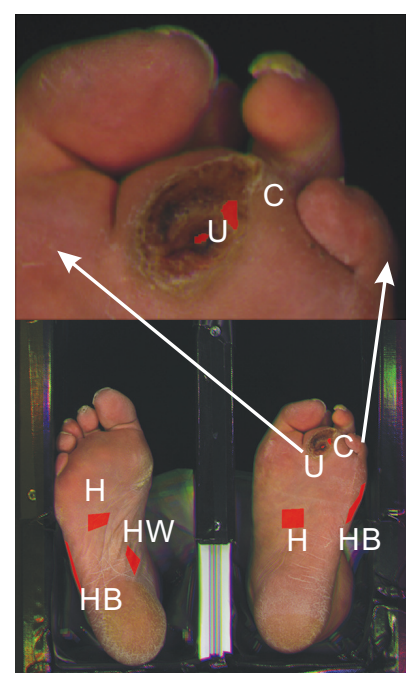

(a) Annotation on Patient 01

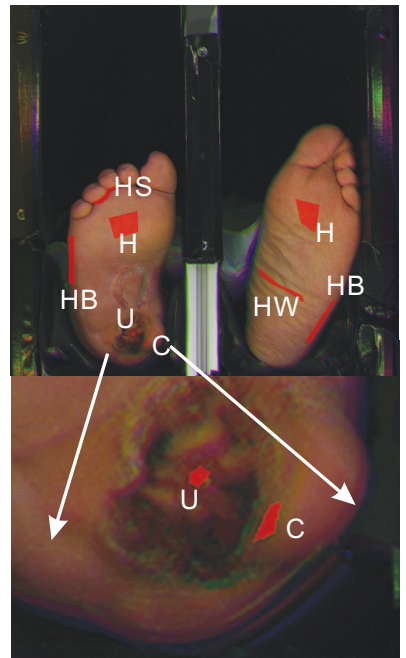

(b) Annotation on Patient 02

Figure 4.10: Annotation with assessment on pseudo RGB color images of diabetic foot. The three color channels in the pseudo RGB color correspond to images taken with filters centred at $650 \mathrm{~nm}(\mathrm{R}), 550 \mathrm{~nm}(\mathrm{G})$ and $430 \mathrm{~nm}(B)$, respectively. The assessment in figures above are presented in a shortened form for illustration purpose: ' $\mathrm{H}$ ' for 'Healthy', 'HS' for 'Healthy Shadow', 'HB' for 'Healthy Border', 'HW' for 'Healthy Wrinkle', 'U' for 'Ulceration' and ' $\mathrm{C}$ ' for 'Callus'. The arrows show a zoom-in for the region with foot complications. 
map with the subjective selection.

\section{Dataset Construction and Summary}

To summarise, 480 regions of interest (ROIs) were annotated from 63 patients with 129 'Healthy', 131 'Healthy Border', 69 'Healthy Shadow', 40 'Healthy Wrinkle', 62 'Callus', 29 'Ulceration', 11 'Rest', 6 'Fissure', 2 'Redness' and 1 'Blister'.

The data from 63 patients were randomly sampled into 2 groups, training set (data from 42 patients) and testing set (data from 21 patients). The summary of the dataset for training and testing can be found in Table 4.1.

\subsubsection{The Design of Pixel Classifiers}

The design of a classifier involves two aspects [86]: $a$ ) selection of the model of classifier, and $b$ ) training the parameters of classifiers via validation.

The optical filters mounted in our experimental setup were chosen through filter selection with Quadratic Discriminant Classifier (QDC) on spectral data of skin spots from diabetic foot acquired with a spectrometer in our previous study [40]. We also concluded that the performance of QDC always outperformed Linear Discriminant Classifier (LDC) [40]. To be consistent, QDC was chosen as the classifier to be trained and evaluated in this study. The more complex classifiers, such as support vector machine or Parzen, were out of consideration, taking the size and the complexity of the dataset into account. Besides, the long time consuming and computation occupancy for the classifier training and evaluation will also hinder the selection of these complex classifiers for a future real-time telemedicine system.

\section{Front-end Pixel Classifier Performance Evaluation}

As a front-end design, we intended to investigate the ability of our pixel classifiers to distinguish a pixel with or without diabetic foot complications. So, when checking into the classification results, all the 'Healthy' classes are merged as one 'No-Complications' and all the other classes (presence with different foot complications) as one 'Complications' class. This formed a binary classification problem for the evaluation of the classifier performance.

The classification results can be presented in a confusion matrix, which reports the counts of the correct and incorrect detection from every class (Tab. 


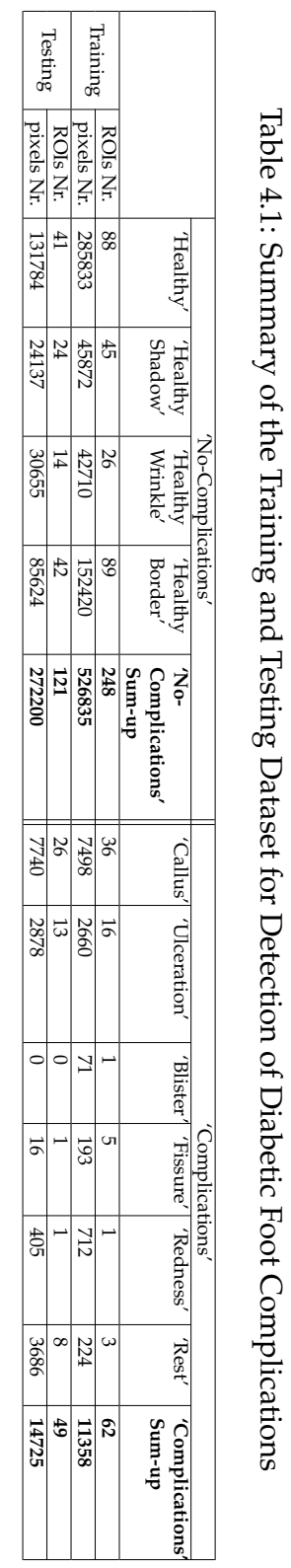


4.2) [107]. The counts are referred to as the numbers of true positive, true negative, false positive and false negative. The confusion matrix allows a more detailed analysis of the performance of the classification rather than a proportional representation.

Based on the confusion matrix, some useful statistical measures for classifier performance evaluation, can be derived [107]:

- Sensitivity, which is also referred to as true positive rate, represents, in our case, the ratio of the pixels with 'Complications' that are correctly identified. Sensitivity indicates the ability of the trained classifier of detecting 'Complications'.

- Specificity represents, in our case, the ratio of the pixels with 'NoComplications' that are correctly identified. This indicates the ability of the trained classifier of detecting 'No-Complications'.

- Positive Predictive Value (PPV): the ratio of the pixels that are identified as 'Complications' that are really 'Complications'.

- Negative Predictive Value (NPV): the ratio of the pixels that are identified as 'No-Complications' that are really 'No-Complications'.

- Accuracy: the ratio of the pixels that are identified correctly over the entire dataset for testing.

Among the five measures, sensitivity and specificity are intrinsic of the classifiers, which will not be influenced by the class prevalence. On the other hands, the other three measures, namely PPV, NPV and Accuracy, depend on not only the intrinsic of the classifiers but also the class prevalence [107]. It can be misleading to apply PPV, NPV or Accuracy in low-disease-prevalence experiment, as our case. As such, the sensitivity and specificity will be used as the measures of the performance of the trained classifiers in this study.

Besides, Receiver Operating Characteristic (ROC) Curve is a long used technique for visualisation and selection of classifiers based on their performance. The ROC curve displays a trade-off between the true positive rates (sensitivities, as the $y$-axis) and the false positive rates ( 1 - specificity, as the $x$-axis) over a series of cutoff points of the test values [108]. It is helpful for determining the optimal cutoff point of the test values for the least misclassification of the two classes. Since ROC curve is based on the sensitivity and specificity, it is also independent of the class prevalence. Area under the ROC curve (AUC) summarises the entire location of the ROC curve, which provides a simple but 
effective way to combine the sensitivity and specificity as a measure of the classifier performance [109].

Table 4.2: The definition of statistical measures based on the confusion matrix [107]

\begin{tabular}{|c|c|c|c|c|}
\hline & \multicolumn{2}{|c|}{ Estimated Labels } & \\
\hline & & 'No-Complications' & 'Complications' & \\
\hline \multirow{2}{*}{ 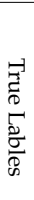 } & 'No-Complications' & True Negative (TN) & False Positive (FP) & $\begin{array}{c}\text { Specificity = } \\
\sum \mathrm{TN} \\
\sum \mathrm{TN}+\sum \mathrm{FP}\end{array}$ \\
\hline & 'Complications' & False Negative (FN) & True Positive (TP) & $\begin{array}{c}\text { Sensitivity = } \\
\sum \sum_{\mathrm{TP}} \\
\sum \mathrm{TP}+\sum \mathrm{FN}\end{array}$ \\
\hline & & $\begin{array}{c}\text { Negative Predictive }= \\
\qquad \frac{\sum \mathrm{TN}}{\sum \mathrm{TN}+\sum \mathrm{FN}}\end{array}$ & $\begin{array}{c}\text { Positive Predictive }= \\
\qquad \begin{array}{c}\sum \mathrm{TP} \\
\sum \mathrm{TP}+\sum \mathrm{FP}\end{array}\end{array}$ & $\begin{array}{c}\text { Accuracy }= \\
\sum \mathrm{TP}+\sum \mathrm{TN} \\
\sum \mathrm{TP}+\sum \mathrm{FP}+\sum \mathrm{TN}+\sum \mathrm{FN}\end{array}$ \\
\hline
\end{tabular}

\section{Experiments for Pixel Classifier Designing}

The achieved dataset were grouped in its feature space according to four schemes:

- Data Scheme 1: Data acquired with the 7 filters selected in our previous study plus the distance map (8 features extracted);

- Data Scheme 2: Data acquired with the 9 filters in the primary set plus the distance map (10 features extracted);

- Data Scheme 3: Data acquired with the 9 filters in the secondary set plus the distance map (10 features extracted);

- Data Scheme 4: Data acquired with all the 18 filters in both filters sets plus the distance map (19 features);

The incidence of the 'Healthy' and its subclasses are far more frequent than that of the other classes. $98 \%$ of objects in the training set belong to 'NoComplications' and $95 \%$ in the testing set. Using the classes frequencies as the prior probabilities, the classifiers may tune themselves to obtain higher accuracy. This may cause the true negative rate to be high while the true positive rate to be quite low or even $100 \%$ wrongly classified. It means that a very high specificity but pretty low sensitivity will be returned, i.e. almost all the diabetic foot complications will be missed. A set of priors for the 10 classes in the dataset was necessary for the pixel classifier design. 
The pre-set priors were changed by varying the total probability of the 'NoComplications' category ( 'Healthy' with its subclasses), $P_{h}\left(P_{h} \in[0,1]\right)$, where the prior of 'Healthy' class accounted for $0.4 P_{h}$ and each of the three subclasses took $0.2 P_{h} .10 \%$ was divided equally for the four classes with the less frequent diabetic foot complications, i.e. $2.5 \%$ for 'Fissure', 'Rest', 'Redness' and 'Blister', respectively. The priors remained unchange for this four classes in our experiment. The rest were taken by 'Ulceration' and 'Callus'. The percentage of these two were set to be equal as $0.5\left(1-P_{h}-0.1\right)$. The values for $P_{h}$ were the cutoff points for displaying our ROC curves.

Generally, three stages were involved for the design of the pixel classifiers, with all the four data schemes, which are:

a) Evaluating QDCs with the training set by 10-fold cross validation to find the proper priors (Fig. 4.12a). 10-fold cross validation while changing the pre-set priors were performed with the training set, and ROC curves were displayed in Fig. 4.12a for all the four data schemes.

b) Use the testing set to evaluate QDCs trained with training set while changing the pre-set priors. The ROC curves were shown in Fig. 4.13a.

c) Determine an optimal cut-off point of $P_{h}^{o}$ based on the ROC curves according to Eq. 4.8 [110], thereby a set of priors for all the classes. With this set of priors, train a QDC with the training set. The testing set were used for the evaluation. Confusion matrix of the classification results were displayed in Tab. 4.4.

$$
\begin{aligned}
P_{h}^{o} & =\underset{P_{h}}{\operatorname{argmin}} d\left(P_{h}\right) \\
& =\underset{P_{h}}{\operatorname{argmin}} \sqrt{\left[1-\operatorname{sensitivity}\left(P_{h}\right)\right]^{2}+\left[1-\operatorname{specificity}\left(P_{h}\right)\right]^{2}}
\end{aligned}
$$

where sensitivity $\left(P_{h}\right)$ and specificity $\left(P_{h}\right)$ are the functions of the test values of $P_{h} . d\left(P_{h}\right)$ represents the distance between every cutoff point on the ROC curves to the left corner of the ROC plot, where has $100 \%$ sensitivity and $100 \%$ specificity.

\subsubsection{Case Studies for Signifying Differences among Diabetic Foot Complications}

The above experiments provide quantitative measures for the evaluation of the front-end pixel classifier design to differentiate pixels with and without any 
diabetic foot complications. Case studies for distinguishing different diabetic foot complications as also conducted, including:

- Evaluation of the performance of the trained QDC classifier in diagnostic foot segmentation per patient in the testing set. Examples of the segmentation results were illustrated in Fig. 4.15.

- Evaluation of the performance of the trained QDC classifier in distinguishing different diabetic foot complications with testing set. The classification results can be found in the confusion matrix (Tab. 4.4). In addition, taking $P_{h}$ as the test variable, ROC curves for each of the six diabetic foot complications versus the 'No-Complications' classes were derived, as shown in Fig. 4.16.

As our final objective of developing the telemedicine system is still to detect different diabetic foot complications, such case studies may help to obtain an insight how the classifiers functioned in differentiating different foot complications and may give some suggestions for the future development of the back-end classification using the spectral images.

\subsection{Results and Discussion}

\subsubsection{Spectral Imaging System Geometrical Calibration}

For the imaging system geometrical calibration, a simple projective transformation estimated using SIFT with RANSAC algorithm was applied. The algorithm automatically detected the corners of the chessboards as the keypoints, which was used as fiducials for image registration. An example of the registration results with the chessboard pattern is illustrated in Fig. 4.11. By simply stacking each three of the registered images as the three colour channels in an RGB image, the quality of the registration could be visually examined. The fiducial registration error was about one pixel $(\approx 0.3 \mathrm{~mm})$.

When applying the registration onto images of patients, the misalignment error, i.e. the target registration error, was larger, especially on the toe regions, which could be $5-10$ pixels $(1.5-3 \mathrm{~mm})$. This misalignment was probably caused by the deviation between the plane of the plantar foot and the plane where the registration was done. As a curved surface, the patient's foot soles were not exactly in the same plane as the one on which the projective transformation was based on. Since we intended to acquire images without any additional 


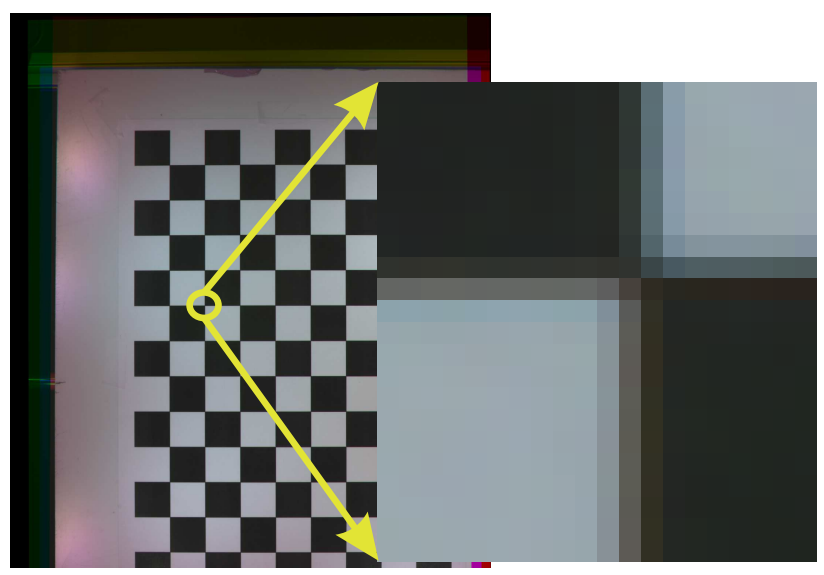

Figure 4.11: Example of an RGB image in which the 3 colour channels are formed by 3 registered image from cameras. On the right side, a zoomed version is given.

pressure on the feet, no sidelong support bars were mounted to force the feet in a standard position and orientation during measurement. This might give the patient some freedom in positioning their feet in the experimental setup. Such drift in the feet position and orientation can also cause deviations in planes. Besides, nonlinear distortion can be another reason of this misalignment.

Currently, as a study for proof of concept, this misalignment was treated to be tolerable.

\subsubsection{The Design of the Pixel Classifiers}

To train the parameters of QDC, which was the classifier model of choice, three stages were conducted with four data schemes of dataset in feature space. The ROC curves (Fig. 4.12a), AUC (Tab. 4.3), sensitivity and specificity were derived from the classification results as the performance measures $a$ ) to compare the performances of the four schemes of the dataset in feature space; $b$ ) to find the optimal cut-off point with the least misclassification; $c$ ) to evaluate the performance of the designed front-end pixel classifier.

As we expected, training QDC with the incidence frequencies of the classes (without preset priors), the false positive rate (1-specificity), was as low as $1.7 \% \sim 2.1 \%$, which meant almost all the healthy pixels were correctly classified. While the sensitivity was around $50 \% \sim 75 \%$ (data with different fea- 


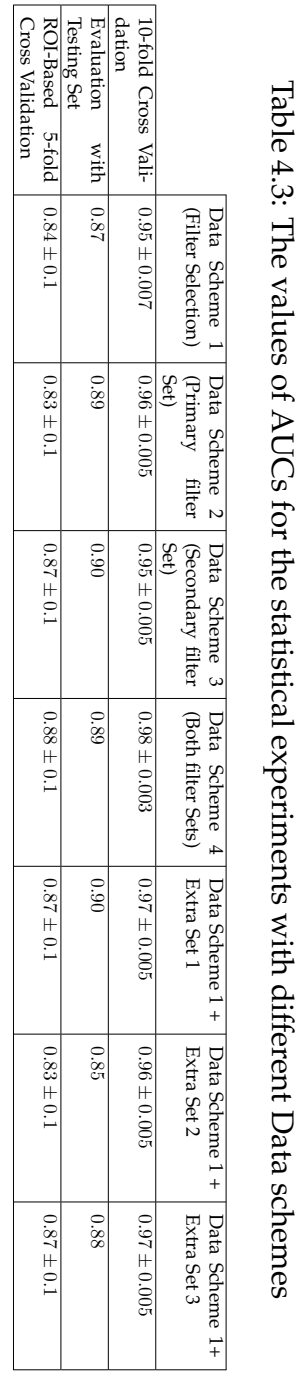


ture spaces returned different results), which indicated half of the pixels with different foot complications were missed.

\section{Choice of the Scheme of Dataset in Feature Space}

Because the experimental setup was built with the optical filters preselected using the skin spectral data measured with a spectrometer, we expected the performance of the spectral images acquired with the set of 7 preselected filters (Data Scheme 1) to be better than the other data schemes (Data Schemes $2-4)$. However, the ROC curves, obtained with 10 -fold cross validation with the training set (Fig. 4.12a), showed that the dataset with all the 19 features (Data Scheme 4) returned the best performance (AUC=0.98), while Data Scheme 1 gave us the worst (AUC=0.95). We gradually added extra filters from the secondary set to the filter selection to see how the addition of extra filters build up the improvement from the original 7 filters up to full monty. Three of additional sets presented results with improvement. These are:

- Extra set 1 contains the images taken with filters at centre wavelengths $480 \mathrm{~nm}(10 \mathrm{~nm}), 880 \mathrm{~nm}(10 \mathrm{~nm})$ and 950nm (25nm).

- Extra set 2 contains the images taken with filters with centre wavelengths $560 \mathrm{~nm}(10 \mathrm{~nm}), 880 \mathrm{~nm}(10 \mathrm{~nm})$ and 950nm (25nm).

- Extra set 3 contains the images taken with filters with centre wavelengths $480 \mathrm{~nm}(10 \mathrm{~nm}), 560 \mathrm{~nm}(10 \mathrm{~nm}), 880 \mathrm{~nm}(10 \mathrm{~nm})$ and 950nm (25nm).

The resulting ROC curves are shown in Fig. 4.12b. When comparing these results, the most important observation was: Data Scheme 1 plus extra set 1 (totally 11 features) or extra set 3 (totally 12 features) almost performed as well as the Data Data Scheme 4, although Data Scheme 1 plus extra set 2 could also improve the performance. We concluded that the filters in extra set 1 should contribute the most in the performance improvement.

Looking at the absorption spectrum and the spectral distribution of the optical filters shown in Fig. 4.1, the absorption spectra of $\mathrm{Hb}$ and $\mathrm{HbO}_{2}$ present with a valley at $480 \mathrm{~nm}$, and even lower absorption for the near-infrared (NIR) region. Such low absorption indicates that less incident light is absorbed by the skin; and the scattering within the skin tissue dominates the reflected light back form the skin. We assumed that less information about the microcirculation and macrocirculation might be revealed in the skin reflectance, while impairment in the blood circulations plays an important role in diabetic foot ulcer 
development [4]. That was the reason the we did not include the absorption valleys in the absorption in the first place for the filter selection.

The improvement of the performance with data acquired within NIR region in detecting diabetic foot complications could also be observed when comparing the ROC curve for the primary set (Data Scheme 2) and that for the selected filters (Data Scheme 1): information acquired with the two supplementary filters at $730 \mathrm{~nm}$ and $800 \mathrm{~nm}$ also helped to improve the performance slightly. The possible reason for the improvement coming from NIR may account for the fact that the irradiance in NIR region has a longer penetration depth $(7 \sim 11 \mathrm{~mm})$ than the light at visible spectral range $(0.2 \sim 2.5 \mathrm{~mm})$ [46].

Possible explanations for the inconsistence between the performance of the filters in the current study and that of our previous study on filter study would be: The illumination sources of our current experimental setup and that for the spectrometer measurement setup are different, not only in the light source emission spectrum but also in the illumination distribution on the target surface. In the spectrometer measurement setup, six illumination fibers encircling one detection fiber were coupled into the reflection probe. The design made sure that the light incident onto the small target skin spot was homogeneous. With a $45^{\circ}$ angle between the probe and the skin surface, the diffuse reflectance was gathered and the specular reflection was eliminated. On the other hand, in our experimental setup, the eight illumination sources were mounted to make sure that each foot may receive light at least from four of the lamps. Although proper calibration with a white reference target was taken, the geometry of the white reference target and the plantar foot surface differed from each other, the compensation with the white reference could not be perfect. Therefore, the illumination on the foot sole could not be perfectly homogeneous.

For local measurement with the spectrometer, the measurement area of the skin was around $60 \mathrm{~mm}^{2}$. Within this small area and under homogeneous illumination, the measurement target could be regarded at the same plane. As to the global measurement with the SI system in our experimental setup, there were risks of self cast shadows for the curved surface of the plantar foot. When the light strikes on the skin, it may be absorbed, transmitted and scattered, irradiation emitted out from skin may change the transmission direction. It was possible that the measured reflected irradiation at one point contained the reflectance of light incident at another position. These effect were neglected in this study. However, for a curved surface as the plantar foot surface, the measured reflectance might be an integration of the information from different skin areas. 
Although the biological mechanism that accounted for the improvement given by the three filters in Extra set 1 was ambiguous at the current stage, Data Scheme 4 and Data Scheme 1 plus Extra set 1 were chosen for the designing the pixel classifiers based on the 10-fold cross validation of the training set.

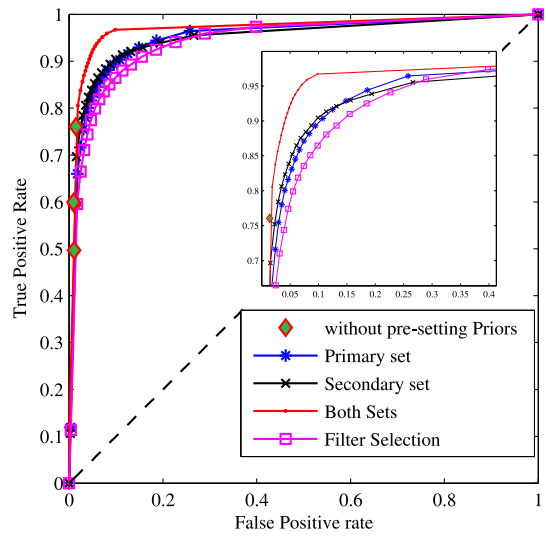

(a)

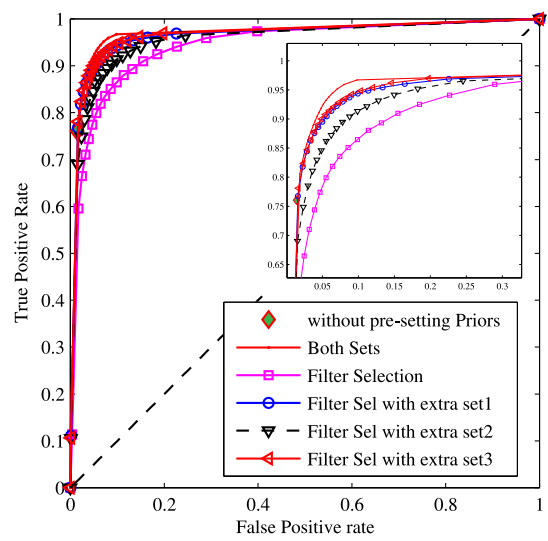

(b)

Figure 4.12: ROC curves for the 10-fold cross validation with the training set. The standard deviations $(0.003-0.007)$ were omitted for illustrative purpose. A zoom-in of the left corner is illustrated in each figure to show the differences between different Data Schemes.

\section{Risk For Overfitting of the $\mathbf{1 0}$-fold cross validation}

The ROC curves presented in Fig. 4.13a illustrated the performance of the trained classifiers with testing dataset. Comparing with the ROC curves in Fig. 4.12a, the performance all dropped. Although the differences between each ROCs were not significant, Data Scheme 4 did not provide the best performance (AUC $=0.89$ ) while Data Scheme 3 (data from the secondary set) presented best performance $(\mathrm{AUC}=0.90)$. This raised a suspicion that there might be some correlations in the dataset, which might cause overfitting in the 10-fold cross validation. When building our dataset, pixels and their neighbours were treated equally in the dataset as objects with the silent assumption that the data in these objects were statistically independent. But unfortunately they may not be. During the process of cross validation, it is possible that the pixels within 
one ROI fell in different folds, which means the data in the training set of cross validation may be correlated with the objects in the validation set. As such, the cross validation had the risk of overfitting. To issue this, an ROI-based

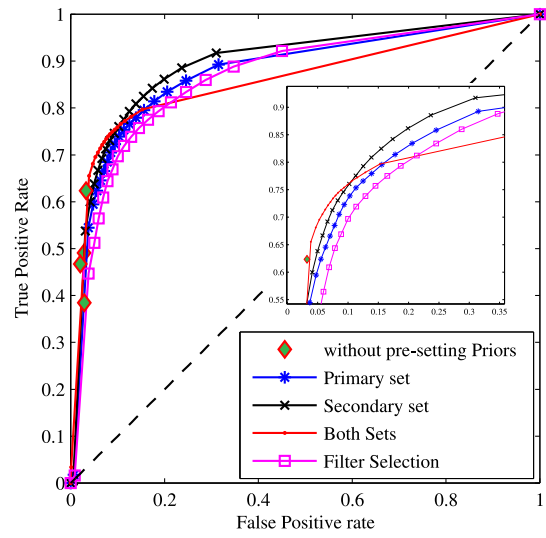

(a)

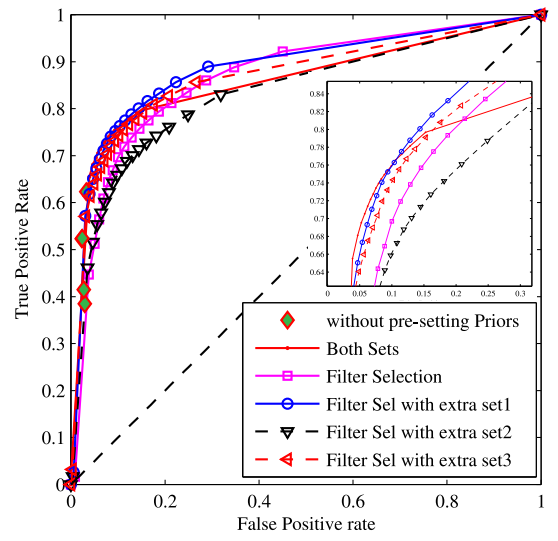

(b)

Figure 4.13: ROC curves for pixel classifier evaluation with the testing set. A zoom-in of the left corner is illustrated in each figure to show the differences between different Data Schemes.

5-fold cross validation was performed according to the following procedure: 1) Merge all the 480 annotated ROIs (both the training set and the testing set) together. As the acquired data set was limited, this could make sure all classes have objects in both training and validation set. 2) Divide 480 ROIs into 5 folds, randomly. 4 of the 5 folds ( $80 \%$ of the ROIs) were taken as the training set, the rest of the ROIs were taken as the testing set. 3) Extract the pixels from the ROIs to construct the training and validation set for classifier training and validation. In other words, the cross validation was performed based on ROIs, classifier training and testing were still conducted with pixels as objects. As such, we could eliminate the correlation between the training set and testing set. The resulted ROC curves are presented in Fig. 4.14.

Comparing the ROC curves in Fig. 4.14 with those in Fig. 4.12, one major observation would be the drops in the performance, which might result from the following possibilities:

- The areas of the ROIs annotated with one of the diabetic foot complica- 


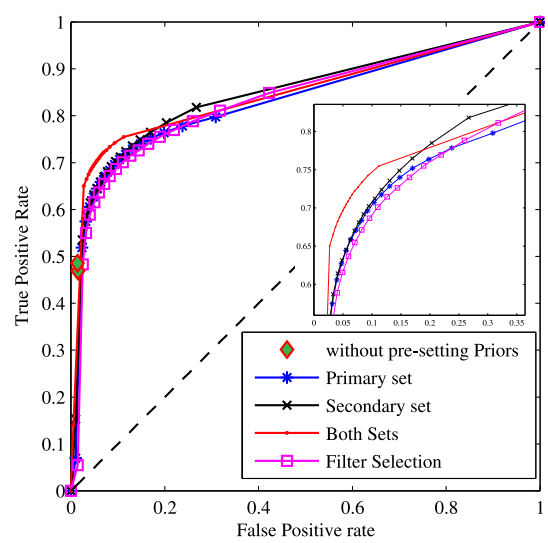

(a)

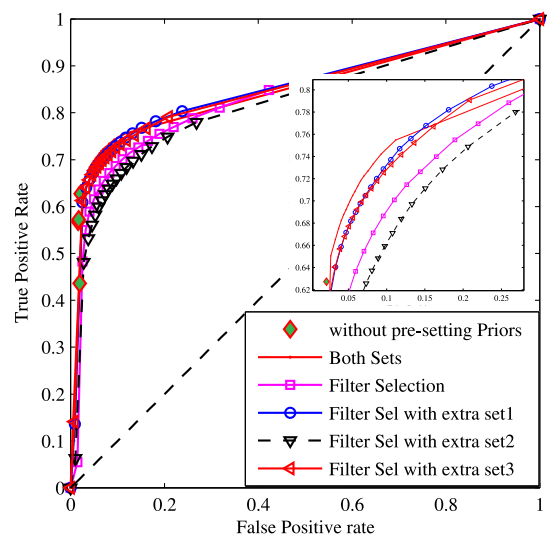

(b)

Figure 4.14: ROC curves for pixel classifier evaluation with 5-fold ROI based cross validation. The standard deviations $(\approx 0.1)$ were omitted for illustrative purpose. A zoomin of the left corner is illustrated in each figure to show the differences between different Data Schemes.

tions differed a lot. Although the training set for this ROI-based cross validation had $80 \%$ of the ROIs, the number of the objects (pixels) in the training set had the chance to be equal to that of the testing set, or less. Lacking of learning examples might cause the performance to drop, especially for the true positive rates.

- The 10-fold pixel based cross validation was overfitted to some extend because of the correlation between training set and validation set.

Although the performance dropped, in Fig. 4.14, Data Scheme 4 still gave the best performance (AUC $=0.88 \pm 0.1$ ); Data Scheme 1 with extra set 1 and extra set 3 kept their ranks $(\mathrm{AUC}=0.87 \pm 0.1)$ and performed almost as good as Data Scheme 4. Thus, the choices of the data schemes, Data Scheme 4 and Data Scheme 1 plus extra set 1, were not influenced. 


\section{Evaluation of Classifier Performance in Detection of Diabetic Foot Compli- cations}

As the Data Scheme 4 and Data Scheme 1 plus extra set 1 performed almost the same, the latter data was applied in this section for the classifier performance evaluation.

Based on the ROC curves for the data of choice (in Fig. 4.12b and Fig. $4.13 \mathrm{~b}$ ), the optimal cutoff point of $P_{h}$ (the prior of the grouped classes 'NoComplications') was calculated according to Eq. 4.8 as $P_{h}^{o}=0.3$. Fixing $P_{h}=P_{h}^{o}=0.3$, a QDC classifier was trained with the data in the training set. The classification results are presented in Tab. 4.4, when evaluating the trained QDC classifier with the testing set.

To investigate the ability of the trained classifier in identifying pixels with or without diabetic foot complications, the evaluation was based on the binary classification problem. The two classes were 'No-complications' and 'Complications'. The grouping of the classes and the classification result are also presented in Tab. 4.4. The sensitivity and specificity of the trained classifier on testing set was $78.8 \%$ and $87.2 \%$.

Examples of the trained classifier on foot diagnostic performance are illustrated in Fig. 4.15a (ii) and Fig. 4.15b (ii), which showed that the trained pixel classifiers did have the ability to detect the diabetic foot complications. There are three major observations, which are:

- Some pixels within one annotation region might be missed. Although the classifiers did identify the annotated regions, the sensitivity would decrease, with a smaller area detected.

- Some pixels from surrounding the annotated 'Complications' were classified as 'Complications'. This might account for the way that our clinical expert performed the annotation to avoid 'mixing' effect. During annotation, we would like to make sure that what annotated in the region were purely the pixels from the assessment score. This did not necessarily mean that other pixel surrounding the annotated ROIs were not.

- Some regions without any diabetic foot complications were classified as 'Complications'. Some of the misclassifications could be excluded by viewing the segmentation results by a clinical expert. For example, the detected region with shape of stripes on the right foot of Example patient 01 in Fig. 4.15a could be excluded. Decision of the exclusion were made base on the location, shapes, sizes, and personal clinical experi- 


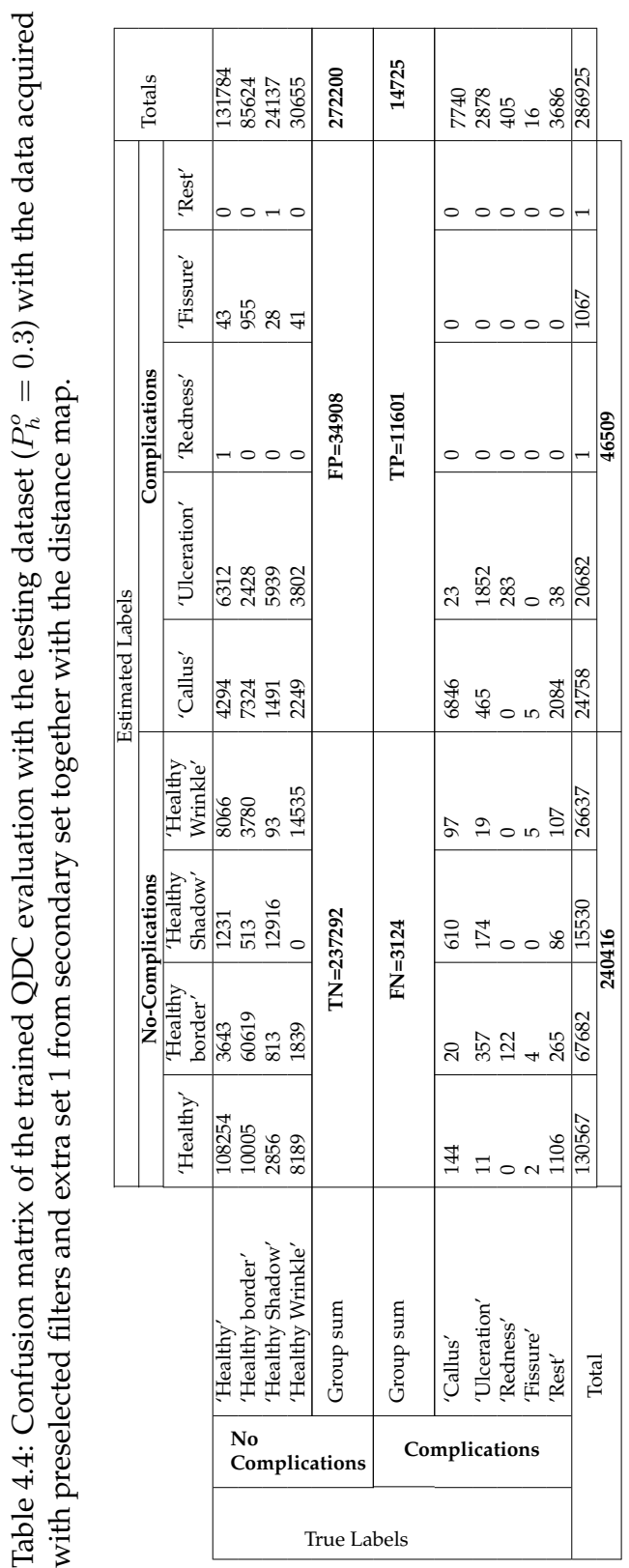


ence. These information should be included in our second layer of the automated system for a future study.

\subsubsection{Case Studies for Signifying Differences among Diabetic Foot Complications}

Although the above evaluations of the classification with the designed QDC presented acceptable results in discriminating skin the diabetic foot complications from healthy skin, we would like to have a check into the current performance in differentiating different diabetic foot complications to provide some suggestions for the future study.

Less pleasant ROC curves ('Callus' versus 'No-Complications' and 'Ulceration' versus 'No-Complications') were illustrated in Fig. 4.16, when evaluating the trained classifier with Data Scheme 1 plus extra data 1 in testing set. The ability of identifying 'Callus' seemed to be higher than that of identifying 'Ulceration', while no pixels from less frequent complications were correctly classified.

Major observations and detailed discussion regarding this two ROC curves in Fig. 4.16, the confusion matrix in Tab. 4.4, and the segmentation results in Fig. 4.15(iii) and Fig. 4.15(iv) are listed below:

- In Tab. 4.4, 88\% 'Callus' and $64 \%$ of 'Ulceration' can be correctly detected.

- Around 36\% of 'Ulceration' were miss classified:

- $16 \%$ of 'Ulceration' were incorrectly classified as 'Callus'. This can be explained with the fact that 'Ulceration' were often surrounded by abundant callus. An example of this are presented in Fig. 4.15b. During annotation for the 'Callus' region from such complications, there might be the risk that skin with blood or other dead tissue were included. This kind of 'mixing' influence could not be avoided.

- It also found that $12 \%$ of 'Ulceration' were classified as 'Healthy Border' and $6 \%$ as 'Healthy Shadow'. As 'Healthy Border' and 'Healthy Shadow' were all located at the regions easily covered by the cast shadows, which might result in low intensities at these regions in the acquired images. As an open wound, the light incident on ulcers can be easily absorbed ( $\mathrm{Hb}$ and $\mathrm{HbO}_{2}$ mainly located in blood), which also resulted lower intensities in the images. 


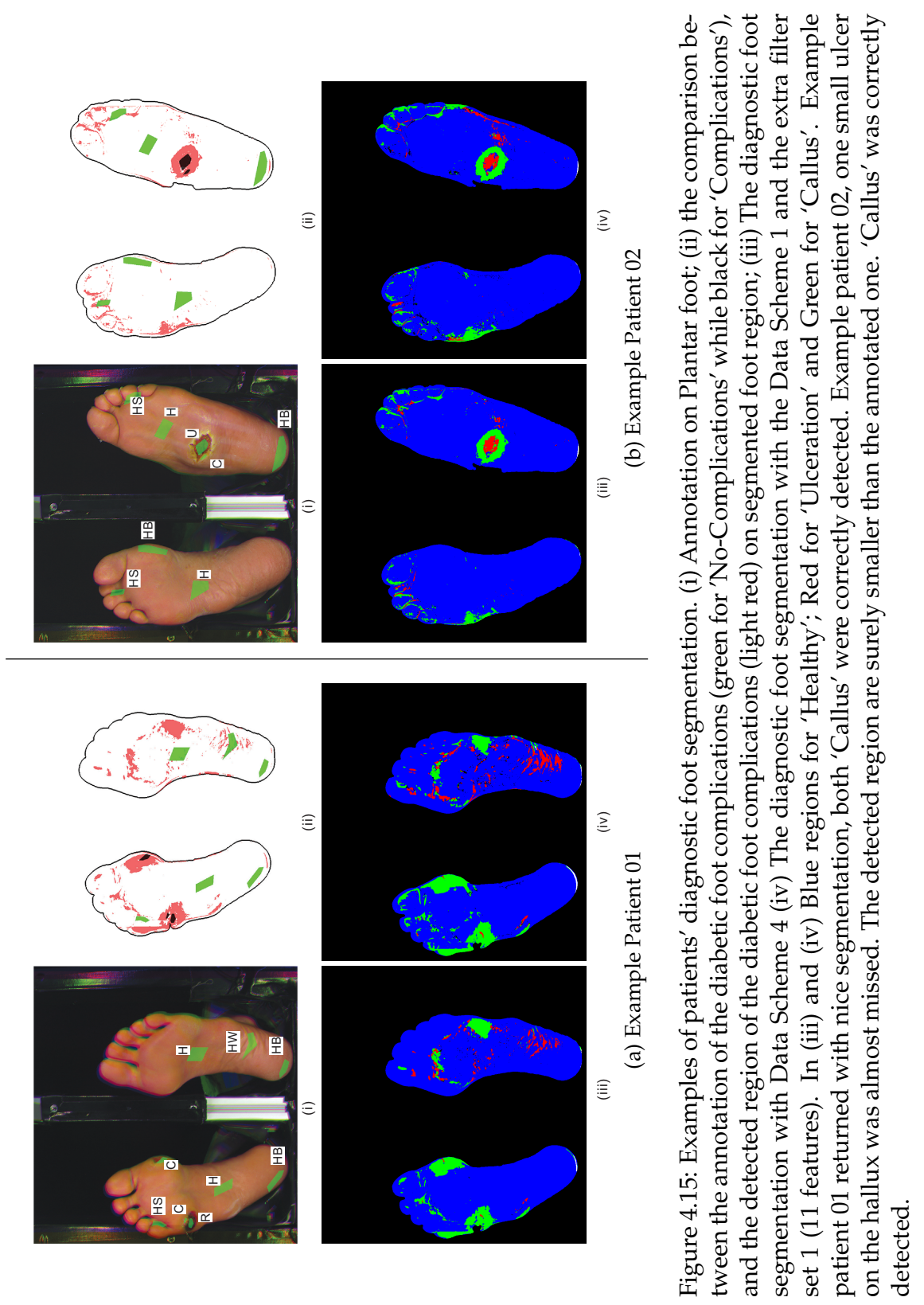




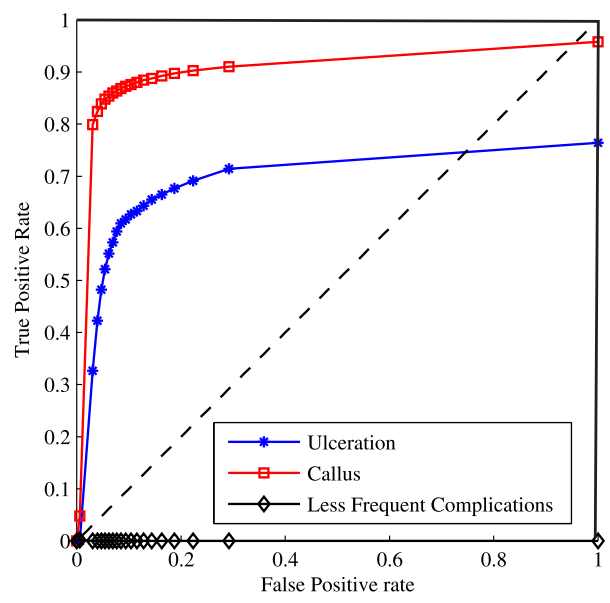

Figure 4.16: ROC curves for pixel classifier evaluation with Data Scheme 1 plus extra data 1 in testing set for assessment of the performance in discriminating different diabetic foot complications.

- $8 \%$ of 'Callus' was misclassified as 'Healthy shadow':

- This phenomenon was out of our expectation. We can not find a proper biological or statistical reason for that currently. Callus is mainly hyperplasia of the stratum corneum, consisting of dead and desquamating cells [111]. Stratum corneum is clear material and only slightly colored. Dry and scaly callus presents as 'white skin'. When light incidents onto the skin, it can only reach up to $2.5 \mathrm{~mm}$ into the skin in visible range and $7 \sim 11 \mathrm{~mm}$ in the NIR range. Because of the abundant callus, the epidermis layer is thicker. Most of the light gets scattered and reflected back before it arrives at the dermis layer. As there is limited melanin in the epidermis layer of plantar foot skin, the light is unlikely to be absorbed much [46]. The attenuation of the incident light mainly results from the considerable scattering in the dry and scaly stratum corneum $[46,111]$. As a result, callus mainly returned with high intensity in the images. On the contrary, 'Healthy Shadow' regions always had low intensities.

- One possible explanation would be that the annotated 'Healthy Shadow' mainly located at the roots of the toes, as shown in Fig. 
4.15 (i). It was ineluctable to include pixels from the toes and pixels under, where were the pressure points for diabetic patients. Thick skin might be resulted from the walking pressure in the regions selected for the 'Healthy Shadow'.

- Low PPVs in classification of 'Callus' and 'Ulceration'

- The PPV in classification of 'Callus' was $37 \%$. The pressure points on the plantar or dry skin presented similar image intensities to the intensities of 'Callus' in the acquired spectral images. The feature 'Distance map' could not help to distinguish these from the abundant callus, because they are actually located at the regions where an abundant callus is likely to develop. The criterion to distinguish thick skin and abundant callus mainly relies on the skin thickness and the experience of the wound specialist.

- The PPV in classification of 'Ulceration' was 9\%. As mentioned, pixels within ulcerated regions usually have lower intensities. Due to different foot poses and different skin colors, pixels with lower intensities were easily to be classified as 'Ulceration'.

- Although the numbers of PPVs were so low as to worry the performance of the designed classifier, these numbers were influenced not only by the intrinsic of the classifier but also the prevalence of the classes. In our case, the data set was so unbalanced that the number of 'No-Complications' pixels were almost twenty times of that of the pixels in 'Complications' classes. In such case, the PPVs and the NPVs could be negligible.

- None of the pixels in 'Redness', 'Rest', 'Blisters' or 'Fissure' were correctly identified. These foot complications happened rarely and so we had limited data in the training set. Taking 'Rest' as example, 3 ROIs in the training set were labeled as 'Rest' from 3 patients, while 8 ROIs in the testing set were labeled as 'Rest' also from 3 other patients. 'Rest' was an assessment contains many different less frequent diabetic foot complications, such as necrosis, crust, scar or some other phenomena that worried the wound consultant. By nature, there were large intra class variation. With such limited information to learn the 'Rest' class pattern, bad performance for this class was as expected. Besides, the selection of these foot complications mainly based on the clinicians' experience. Different clinicians may have different opinions on the assessment. So far, only one 
clinician was doing the live assessment for each patient. So, such bias in the assessment can not be avoided.

\subsubsection{Limitation of the Study}

One limitation of the study was that only one clinician performed in the live assessment, which was taken as the ground truth for the classifier training and testing. Because the personal clinical experience may be limited or specific for each clinical specialist, a bias can be introduced in the live assessment, specially for the less frequent foot complications. In the future, more clinicians should be involved for the live assessment to reduce such bias in live assessment. In the meantime, an investment should be conducted to check the inter-clinician variation.

It also had the limitation in taking the live assessment by visual inspection of the foot skin status as the ground truth. Changes in the spectral information may happen ahead of presence of the diabetic foot complications. Previous studies $[56,57,59]$ indicated that skin spectral information could be used to predict the wound development and healing. These study found that the changes in micro- and macro- circulation might happen days before the wound formation and healing. These changes were beyond the visual inspection but might be detected timely using the spectral information. A longitudinal data acquisition and analysis would be helpful.

\subsection{Conclusion}

In this contribution, an experimental setup with a 9 camera array fitted with 18 filters were built for spectral images acquisition from plantar foot of diabetic patients at high-risk for foot ulceration and amputation. We have demonstrated the utility of the spectral images taken with the experimental setup from 63 subjects with 480 regions annotated with different assessment scores. A methodology combining imaging system calibration, spectral image calibration and foot segmentation, normalisation was proposed, for the purpose of utilising the annotated dataset for the front-end pixel classifier training and evaluation to detect the diabetic foot complications.

With the pixel classifier trained with the available dataset, classification results of a testing set with 21 patients presented an acceptable performance of $78.8 \%$ sensitivity and $87.2 \%$ specificity for discriminating whether the pixels present or absent of diabetic foot complications. This result proves the concept 
that the designed pixel classifier has the ability to detect the diabetic foot complications with the acquired spectral images, which achieved the objective of the front-end pixel classifier designing.

However, $88 \%$ 'Callus' and only $64 \%$ of 'Ulceration' was correctly detected with the trained pixels classifier. This indicated that the capability of the pixel classifier in discriminating the different foot complications was low, the risk of misdetection within 'Complications' was high. A back-end processing using the output of these classifiers will be essential. In the back-end processing, higher level expert knowledge should be exploited to boost the performance.

Further study with the spectra images will focus on enhancing the performance of the front-end classifier and the design of the back-end processing to improve the detection of different diabetic foot complications. These improvements can be achieved by a) Including more patients for the training and testing, covering more variations within classes. $b$ ) Super-resolution to group data with a Gaussian pyramid to eliminate the possible correlation in the data set. c) Perform a more delicate imaging system registration by taking the intrinsic and extrinsic cameras properties into consideration. This may help to Reduce the misalignment and thereby to improve the performance of the classifiers. d) The clinician experience, such as the area and shapes of complication regions should be included in the back-end processing to further filter/improve the diagnostic segmentation. $e$ ) Deriving biological causal parameters from the spectral data measured based on a skin optical model. The biological parameters may also improve the performance, for example by thresholding the thickness of the epidermis layer to distinguish abundant callus and normal thick skin. f) Combination of the other two modalities in our experimental setup, namely infrared thermal imaging and photometric stereo imaging. 



\section{Automatic Detection of Diabetic Foot Complications with Infrared Thermography by Asymmetric Analysis}

\subsection{Abstract}

Early identification of diabetic foot complications and their pre-signs is essential in preventing their devastating consequences such as foot infection and amputation. Frequent, automatic risk assessment by an intelligent telemedicine system might be feasible and cost-effective. Infrared thermography is a promising modality for such a system. The temperature differences between corresponding areas on contralateral feet are clinically significant parameters. This asymmetric analysis is hindered by (i) foot segmentation errors, especially when the foot temperature and the ambient temperature are comparable, and by (ii) different shapes and sizes between contralateral feet due to deformities or minor amputations. To circumvent the first problem, we used a colour image and a thermal image acquired synchronously. Foot regions, detected in the colour image, were rigidly registered to the thermal image. This resulted in $97.8 \% \pm 1.1 \%$ sensitivity, and $98.4 \% \pm 0.5 \%$ specificity over 76 high-risk diabetic patients with manual annotation as reference. Non-rigid B-splines landmarkbased registration solved the second problem. Corresponding points in the two feet could be found irrespective of shapes and sizes of the feet. With that, the temperature difference of left and right feet could be obtained. tics

The contents of this chapter has been submitted to Journal of Biomedical Op- 


\subsection{Introduction}

Diabetes Mellitus (DM) is one of the major health care problems worldwide and continues to increase in population and significance [2]. Foot diseases are common and costly complications of DM. Approximately $15 \%$ to $25 \%$ of patients with DM eventually develop a foot ulcer [3]. This is one of the key complications of DM: if not adequately treated, the risk of amputations and mortality is increased [7]. Early identification and subsequent treatment of diabetic foot complications and its pre-signs, such as ulceration, inflammation, callus formation and blisters, is fundamental in prevention of these devastating consequences. Timing is essential for early identification. However, frequent risk assessment by health care professionals is costly and impractical. Examination by patients themselves has its limitations because of various health impairments caused by DM. Automatic assessment and detection of diabetic foot complications in a noninvasive, noninteractive, and easy to use manner may be a major benefit for foot care. The ultimate objective of our project is to develop an intelligent telemedicine system that can be deployed at the patients' homes or at health centres, for frequent foot assessment in order to detect diabetic foot complications in a timely manner.

\subsubsection{Related Work}

\section{Telemedicine using digital photography}

Frequent foot screening for risk assessment is possible by means of telemedicine systems based on digital photography [19,32]. However, inflammation and infection, which are vicarious markers of diabetic foot complications, are difficult to assess using digital photography [32]. Furthermore, such systems are not yet capable of automatic detection of diabetic foot complications. This limits their application and implementation.

\section{Thermography}

Associations have been found between increased plantar foot temperature and the occurrence of diabetic foot complications [63]. Clinical studies on the homemonitoring of plantar foot temperature have shown that frequent temperature assessment and treatment in case of temperature differences $>2.2{ }^{\circ} \mathrm{C}$, between a foot region and the same region on the contralateral foot, can prevent diabetic 
foot complications $[5,6]$. Thus, thermography is a promising modality for an intelligent telemedicine monitoring system.

The technologies for temperature measurement of the plantar foot fall in three categories: hand-held dermal infrared (IR) thermometers [5,6], IR camera systems $[67,68,70,71,73,74]$, and liquid crystal thermography (LCT) [66,69]. The use of hand-held dermal IR thermometers in the home environment has been validated by randomised controlled trials for the prevention of recurrent diabetic foot ulceration $[5,6]$. The shortcoming of this technology is that the temperature is measured manually on specific spots on the foot. This makes it subjective, and it is impossible to obtain the temperature distribution of the whole foot. Furthermore, this technology misses the opportunity for automatic detection of diabetic foot complications. Compared with LCT, IR camera systems have the advantage of being non-contact, which prevents unwanted pressures and the transmission of pathological organisms [71]. The temperatures of non-contact foot regions, such as the medial arch, can be easily measured with IR camera systems. Additionally, it is capable of measuring the dorsal side of the foot as well. As such, IR camera systems show greater potential for telemedical applications and they will be the focus of this article.

\section{Asymmetric Analysis}

The methodology for attaining temperature differences between corresponding areas on the left and right feet, can be referred to as 'asymmetric analysis' $[73,74]$. Generally, three steps are involved:

- Foot Segmentation: Extract the left foot and the right foot from the background.

- Feet Registration: Register the two feet to associate areas of one foot with corresponding areas of the contralateral foot.

- Detection: Compare the temperature of associated areas: if the difference between the temperatures of two associated areas is larger than a certain threshold, one of these two areas is assumed to be at risk.

Kaabouch and her colleagues conducted studies, using thermal images to detect neuropathic ulceration, combining a genetic automatic thresholding algorithm with 'asymmetric analysis' [73] and with 'line scanning' [74]. The authors noticed that the asymmetric analysis tended to find false abnormal areas when the left and right feet had different sizes and shapes. In these 
cases, non-corresponding areas were compared. The 'line scanning' algorithm was proposed to overcome this limitation. Nevertheless, their methodologies were only validated with people whose left and right feet had similar sizes and shapes. The thermal images of the feet could show differences in shapes and sizes between the left and right feet, but this was caused by the perspective projection distortion that occurred during imaging when the feet were not properly aligned.

However, differences in foot shape and size between left and right are not always the result of projection distortion. Foot deformations and/or minor amputations frequently cause morphological differences between left and right feet in patients at high-risk for developing diabetic foot complications [7]. Additionally, in case foot temperature is similar to ambient temperature, e.g. the cold toes shown in Fig. 5.2a, the genetic automatic thresholding techniques cannot accurately detect the foot boundaries [112]. The methodology proposed in this article aims to overcome these shortcomings.

In this study, the novel aspects of asymmetric analysis for automatic detection of diabetic foot complications are as follows:

- Accurate foot segmentations in the thermal images were obtained even when there was no clear thermal contrast between foot and background. This was due to the accompanying colour images that guided the segmentation.

- Non-rigid landmark-based registration with B-splines [113-115] between the left and right feet regions was conducted successfully. This established correct associations between corresponding areas in the left and right feet regardless of the foot poses, positions, shapes, or sizes, and even with amputated parts.

- The image analysis was applied to a group of high-risk diabetic patients that showed significant asymmetries between left and right feet, rather than to subjects with healthy feet.

\subsubsection{Outline of the Article}

In Section 5.3, the experimental setup for acquiring thermal images and colour images from patients with DM, the measurement procedure, patient recruitment, and data collection are described. The methodology of conducting asymmetric analysis to detect foot complications using the assistance of colour images and non-rigid landmark-based registration with B-splines are introduced 
in Section 5.4. Analysis, results, and discussion follow in Section 5.5. Finally, the conclusions and future plans are presented in Section 5.6.

\subsection{Materials and Measurements}

An experimental setup, consisting of an IR camera, FLIR SC305, and a commercial digital RGB camera, Canon EOS 40D, has been built as shown in Fig. 5.1. The IR camera has a resolution of $320 \times 240$ pixels and is placed at a distance of $860 \mathrm{~mm}$ from the measurement plane, covering a field of view of $400 \times 350 \mathrm{~mm}^{2}$. It provides a pixel distance on the foot soles of about $1.25 \mathrm{~mm}$. The digital camera is placed at the same distance, covers the same field of view, and acquires images containing $1936 \times 1288$ pixels (pixel distance: $0.2 \mathrm{~mm}$ ).

Prior to applying the experimental setup for any clinical tests, the uniformity of the IR camera response was characterised with the help of six thermal references. The reference elements were designed to provide homogeneous temperature distributions over their visible fronts. For calibration, these elements were heated to different, accurately known, constant temperatures in the expected temperature range for foot soles $\left(20^{\circ} \mathrm{C}-38^{\circ} \mathrm{C}\right)$. The maximal difference of temperature readings of the camera of two isothermal references, one far left and one far right in the image plane, is $\pm 0.25^{\circ} \mathrm{C}$ for $4 \times 4$ pixel areas (circa $5 \times 5 \mathrm{~mm}^{2}$ ).

Patients were recruited from the multidisciplinary diabetic foot clinic of the Hospital Group Twente, Almelo, the Netherlands. The patients included in this study were all diagnosed with DM and showed diabetic foot complications, e.g. callus, blisters, redness, ulceration, or had a history of ulceration. The average age of the 76 included patients, 64 male and 12 female, was 66 years $(\mathrm{SD}=12)$. Among them, there were 7 patients with Type I DM and 69 patients with Type II DM.

All patients were asked to remain in a seated position with bare feet for 510 minutes before the measurements. This was to achieve equilibrium of the foot temperature. The feet were then placed on the foot support of the experimental setup. A hospital cloth was placed over the legs of the patients for hygiene reasons and one piece of black cloth was draped over the shield of the setup to block external light entering the setup and to provide a homogeneous background. During thermal imaging, all illumination sources inside the experimental setup were turned off and turned back on during the acquisition of colour images with the digital camera. A 30-minute interval was assured between measurements of different patients to eliminate heat residue from the 


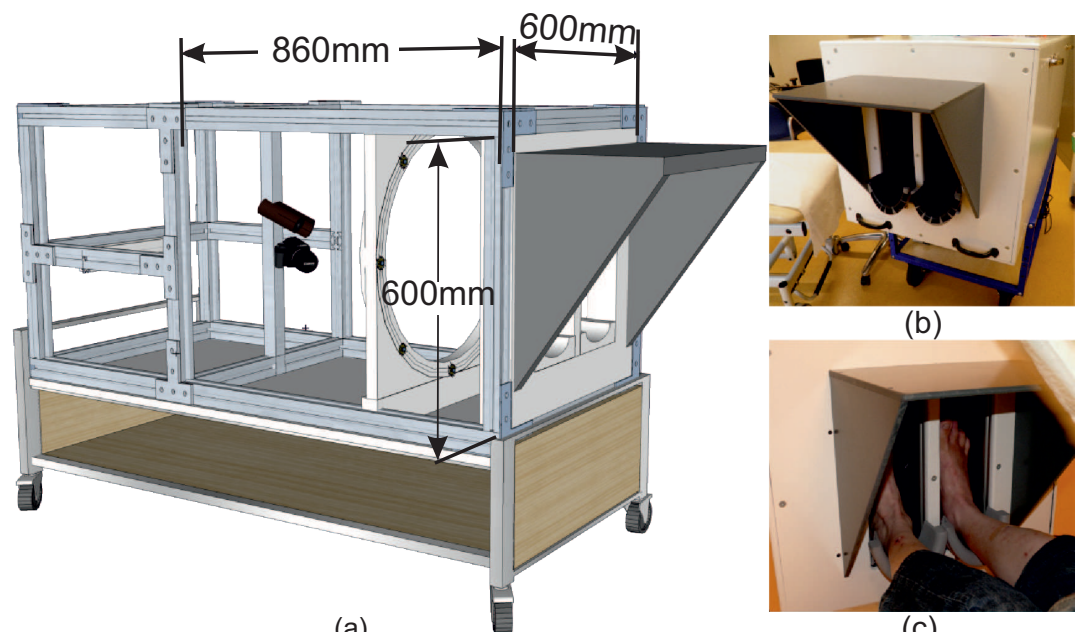

(a)

(c)

Figure 5.1: The experimental setup for foot scanning. (a) the schematic of the experimental setup with 2 cameras installed, which are the thermal camera (upper) and the RGB camera (lower). (b) The appearance of the experimental set; (c) Positioning of patients' feet during measurement.

illumination or from the body of the previous patient.

For each patient, a live assessment form of the plantar surface of both feet was completed by wound care specialists. This live assessment was used as a reference for the validation of the automatic detection. Examples of the acquired thermal and RGB images are given in Fig. 5.2.

\subsection{Methodology for Automatic Detection of Dia- betic Foot Complications}

\subsubsection{Overview of the Proposed Methodology}

The proposed methodology for patient specific image analysis to automatically detect diabetic foot complications is illustrated in Fig. 5.3.

In our experimental setup, the digital RGB camera and the IR camera were mounted in a way such that they had the same field of view, but at slightly different view points. Before the actual deployment of the device, the parame- 


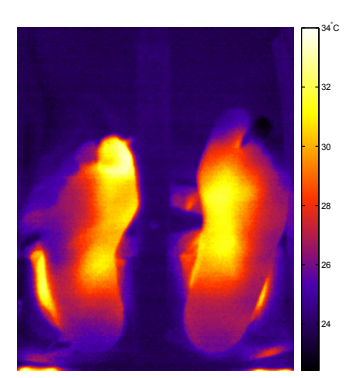

(a) Example 1

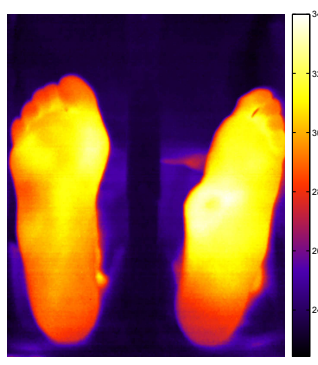

(b) Example 2

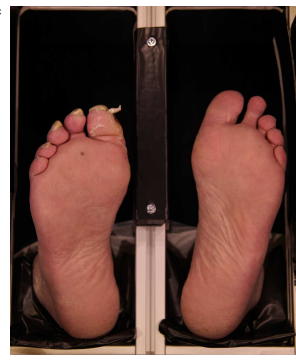

(c) RGB Image for 5.2a

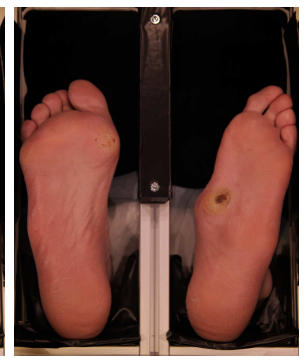

(d) RGB Image for $5.2 b$

Figure 5.2: Acquired thermal images with their RGB image. Example 1: patient has cold toes, which have temperatures comparable with the background. Ankles have temperatures comparable with the feet. The ankles are also visible in the RGB image. Example 2: one of the ankles is not visible in the RGB image but visible in the thermal image (left foot).

ters that were needed to register the colour images on the thermal images were determined. For this registration, a 2D projective transformation (a homography) has been used. The 8 parameters of this transform were obtained from 12 control points, which were all located in a single plane in front of the cameras at a distance that matched the mean distance between cameras and the plane of the foot soles.

After image acquisition, the next step was the segmentation of the feet in the colour images. Due to the fact that a foot usually has a lower temperature than other body parts, and that a foot may have a temperature that is comparable with the ambient temperature, the segmentation can be difficult to perform directly in the thermal image. Thus, foot segmentation in the colour images was preceded to assist the segmentation in the thermal images. Details about foot segmentation in the colour images can be found in Section 5.4.2.

The projective transformation mapped the foot segments from the RGB image plane to the IR image plane. However, this was only an approximate registration as the patient's foot soles were not exactly in the same plane as the one on which the projective transform was based. A further registration optimisation was necessary. Details of the optimisation method can be found in Section 5.4.3.

The performance evaluation of the segmentation in the colour images and that in the thermal images was achieved through comparison with manually- 
segmented references provided by one of the researchers. The method for manual segmentation with custom-made software has been described elsewhere [116].

The last step was to conduct asymmetric analysis to detect the temperature differences of contralateral regions on left and right feet. To facilitate this, a good registration between left and right feet, irrespective of feet positions, poses, shapes or sizes, was essential. Non-rigid landmark-based registration was performed for this purpose. Details of this part can be found in Section 5.4.4.

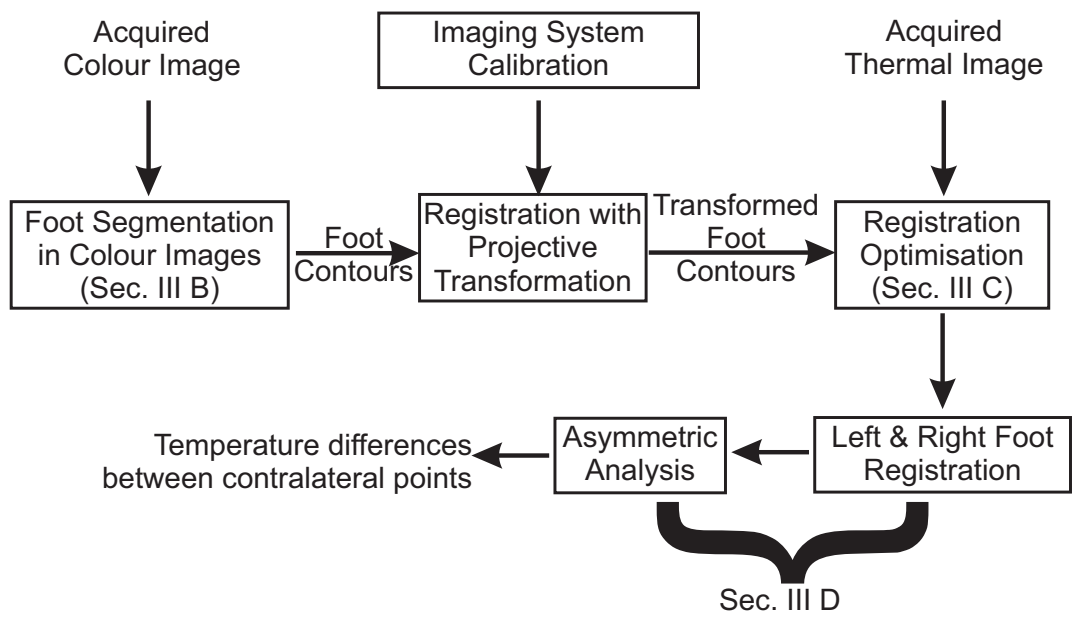

Figure 5.3: Schematic flowchart of the proposed methodology for automatic detection of diabetic foot complications.

\subsubsection{Foot Segmentation in Colour Images}

The aim of the foot segmentation with acquired colour images is to determine whether a colour pixel is on a foot or on the background.

\section{Colour Spaces}

Segmentation in colour images is not restricted to the RGB colour space. A wide variety of colour spaces have been proposed and applied for colour im- 
age segmentation for different applications. The performance of an image segmentation procedure is known to depend on the choice of the colour spaces. RGB is the most common colour space for storing and representing images. Any other colour space can be obtained by a linear or a non-linear transformation from RGB. It has been observed that skin colours differ more in intensity than in chrominance [117]. Thus, it is common practice to ignore the luminance in skin colour detection. Although many studies have been done in segmentation with different colour spaces, it is hard to make a conclusion which colour space is best. Most studies aimed to find the most appropriate colour space for their specific problem [118]. In this study, we performed segmentation on the 6 colour spaces that are commonly used in human skin detection: RGB, normalised RGB (rgb), RGB-ratio, HSV, CIE L` $a^{\star} b^{\star}$ and YCbCr [117].

\section{Machine Learning for Colour Image Segmentation}

Machine learning techniques play an important role in image segmentation. The goal is to partition the image plane into $K$ disjoint regions. Suppose that the 3 colour channels of the $\ell$-th pixel in the image are represented by a $3 \mathrm{D}$ vector $\mathbf{x}_{\ell} \in \mathbb{R}^{3}$, and suppose that the image contains $L$ pixels, so that $\ell=$ $1,2, \cdots, L$. The segmentation is accomplished by assigning to each pixel $\mathbf{x}_{\ell}$ a class label $k_{\ell}$. The set of possible class labels is $k_{\ell} \in\{1, \cdots, K\}$. Each class corresponds to a region.

The techniques for machine learning can be divided into two groups: supervised and unsupervised learning [86]. Supervised learning requires the availability of a set of samples drawn from the data. These samples should be independent and representative, and their true class labels should be available. This training set is used in machine learning to find an assignment of labels to data with unknown classes.

In unsupervised learning, the class labels in the training set are missing. The goal of the techniques in this category is to determine the 'natural groups' or intrinsic clusters of the unlabeled vectors (image pixels). Each resulting cluster contains vectors that are mutually similar, and are dissimilar to the vectors of other clusters. Different clustering algorithms may lead to different clusters [86].

In this study, all patients had a wide variety in skin health status, and accordingly in colours of the skin. It is difficult to cover all the possible variations in a training set. In contrast, unsupervised learning techniques are able to tune themselves according to the information from each individual image. Thus, instead of supervised learning, unsupervised learning was selected. Two com- 
mon techniques in this category were chosen in this study, K-means clustering and Expectation-Maximisation (EM) clustering.

K-means clustering is one of the simplest unsupervised learning algorithms, aiming to define $K$ centroids for $K$ clusters. $K$ is predefined. At the initialisation step, the $K$ centroids are randomly picked. A binding step follows to associate each object in the training set to the nearest centroid. When no point is pending, the $K$ centroids are recalculated. With the new $K$ centroids, a new binding step is done. This procedure iterates until the $K$ centroids do not move any more. K-means minimises the sum of the distances over all objects in $\mathbf{X}$ to the nearest centre $\mathbf{m}_{k}$, and the algorithm converges to a (local) minimum [86].

The EM algorithm is a popular technique in unsupervised learning [86]. A probabilistic model $f$ is used to estimate the joint probability distributions $p(\mathbf{x}, k \mid f)$ iteratively, where $\mathbf{x}$ is a pixel and $k$ the associated class label. We used the EM algorithm for the case that the model is a mixture of Gaussians. Each class is represented by a Gaussian density $N\left(\mathbf{x} \mid \mathbf{m}_{k}, \mathbf{C}_{k}\right)$ with mean $\mathbf{m}_{k}$ and covariance matrix $\mathbf{C}_{k}$. The prior probabilities of the classes are denoted by $\pi_{k}$. We implemented two versions of the EM algorithm; one with class independent covariance matrices, i.e. $\forall k: \mathbf{C}_{k}=\mathbf{C}_{0}$, and one with class dependent matrices. The first one is referred to as EM-LDC (linear discriminant classifier); the second one as EM-QDC (quadratic discriminant classifier):

Algorithm: EM for image segmentation

- Inputs: Data set with $L$ pixels (3D vectors) $\mathbf{x}_{\ell}$. Desired number of clusters $K$.

- Output:

- A model $f$ consisting of the parameters of the mixture of Gaussians, i.e. for $k=$ $1, \cdots, K: \mathbf{m}_{k}, \mathbf{C}_{k}, \pi_{k}$.

- For each pixel $\ell$ and each class $k$, an ownership variable $y_{\ell, k}$ such that $\Sigma_{k}\left(y_{\ell, k}\right)=1$. The ownership indicates to what degree pixel $\mathbf{x}_{\ell}$ is attributed to cluster $k$.

- Initialisation:

- Determine $K$ centers $\mathbf{m}_{k}$ from the $L$ pixels using the K-means algorithm [86].

- Initialise the ownerships $y_{\ell, k}$ as the distance of pixel $\ell$ to the class centre $\mathbf{m}_{k}$ relative to the sum of distances to all class centres.

- Loop while the model $f$ improves:

- For all $k$ : (re-)estimate the prior probabilities:

$$
\pi_{k}=\frac{1}{L} \sum_{\ell=1}^{L} y_{\ell, k}
$$


- For all $k$ : (re-)estimate the means:

$$
\mathbf{m}_{k}=\frac{1}{\pi_{k} L} \sum_{\ell=1}^{L} y_{\ell, k} \mathbf{x}_{\ell}
$$

- For all $k$ : (re-)estimate the covariance matrices:

$$
\mathbf{C}_{k}=\frac{1}{\pi_{k} L} \sum_{\ell=1}^{L} y_{\ell, k}\left(\mathbf{x}_{\ell}-\mu_{k}\right)\left(\mathbf{x}_{\ell}-\mu_{k}\right)^{T}
$$

- Equalise the covariance matrices (EM-LDC only):

$$
\begin{gathered}
\mathbf{C}_{0}=\sum_{k=1}^{K} \pi_{k} \mathbf{C}_{k} \\
\mathbf{C}_{k}:=\mathbf{C}_{0}
\end{gathered}
$$

- For all $k$ and all $\ell$ : re-estimate the ownerships:

$$
y_{\ell, k}=\frac{\pi_{k} N\left(\mathbf{x}_{\ell}, \mathbf{m}_{k}, \mathbf{C}_{k}\right)}{\sum_{m=1}^{K} \pi_{m} N\left(\mathbf{x}_{\ell}, \mathbf{m}_{m}, \mathbf{C}_{m}\right)}
$$

There are two different regions in this application, foreground (foot regions) and background. Consequently, the number $K$ of clusters should also be set to 2. However, since the background was made up by different parts, it could be advantageous to increase this number. It was set to 2, 3 and 4, respectively. In case of more than two clusters, only one of them was identified as foreground (foot region). Post processing of the segmentation was performed by morphological operations. Small objects either in the foreground (foot area) or in the background were removed.

All the image processing and pattern classification were performed on the Mat l ab R2012b platform with an additional toolbox PRtools V5 [86] in 64bits Windows 7.

\section{Segmentation Evaluation}

To select the number $K$ of desired clusters, and the most suitable algorithm (K-means, EM-LDC, or EM-QDC), a quantitative measure of the segmentation performance is necessary. Two measures were included in the evaluation step for the segmentation:

- Sensitivity and Specificity

Sensitivity and specificity are measures of the performance of a two class 
classification algorithm. Sensitivity, in our case, measures the ratio of the pixels inside the feet regions that are correctly labeled. Sensitivity represents the ability of the segmentation method in finding the feet. Specificity, on the other hand, measures the portion of negatives (the background, in our case) that are correctly identified.

$$
\begin{aligned}
& \text { Sensitivity }=\frac{\left|F_{R} \cap F_{S}\right|}{\left|F_{R}\right|} \\
& \text { Specificity }=\frac{\left|B_{R} \cap B_{S}\right|}{\left|B_{R}\right|}
\end{aligned}
$$

where $B_{R}$ and $F_{R}$ represent the background and foreground of the reference image, while $B_{S}$ and $F_{S}$ donate the background and foreground according to the algorithm under test. $|\cdot|$ is the set cardinality.

- Mean Distance Error of Wrongly Classified Area $\left(\overline{\mathrm{D}}_{\mathrm{EA}}\right)$

This reflects the cost of wrong segmentation. For each pixel on the image, the Euclidean distance was calculated to the nearest foot contours resulting in a distance map $\mathbf{D}_{\text {map }}$ as shown in Fig. 5.4. The distance of each pixel that was wrongly assigned, was summed up and averaged to get the mean distance error $\left(\overline{\mathrm{D}}_{\mathrm{EA}}\right)$ :

$$
\overline{\mathrm{D}}_{\mathrm{EA}}=\frac{\sum_{\mathrm{Nwb}} \mathbf{D}_{\text {map }} \mathbf{W}_{B}+\sum_{\text {Nwf }} \mathbf{D}_{\text {map }} \mathbf{W}_{F}}{\mathrm{Nwb}+\mathrm{Nwf}}
$$

where $\mathbf{W}_{B}$, represents a binary map containing the foot pixels that are wrongly assigned to the background i.e. $F_{R} \cap B_{S}$ with $\mathrm{Nwb}=\left|F_{R} \cap B_{S}\right|$ and $\mathbf{W}_{F}$ represents a binary map containing the background pixels that are wrongly assigned to the foot, i.e. $B_{R} \cap F_{S}$ with Nwf $=\left|B_{R} \cap F_{S}\right|$.

\subsubsection{Registration Optimisation}

Since we intended to acquire a thermal image without any additional pressure on the feet, no sidelong support bars were mounted that would force the feet in a standard position and orientation in the imaging system. As a result, the surfaces of the foot soles only approximately coincided with the plane that was used for the projective registration. Deviations from that plane caused misalignments between colour and thermal images. The main disagreement 


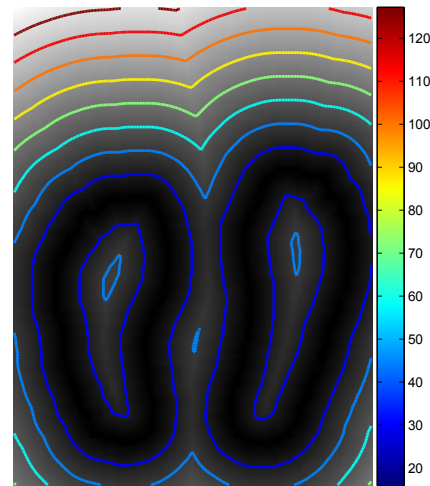

(a) $D_{\text {map }}$ of Example 1

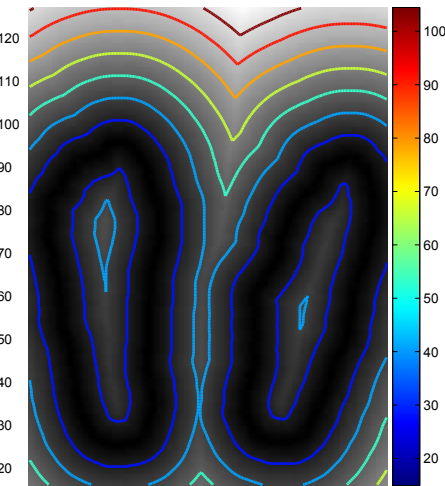

(b) $D_{\text {map }}$ of Example 2

Figure 5.4: Examples of Euclidean distance map of the reference foot contours (Unit: pixel).

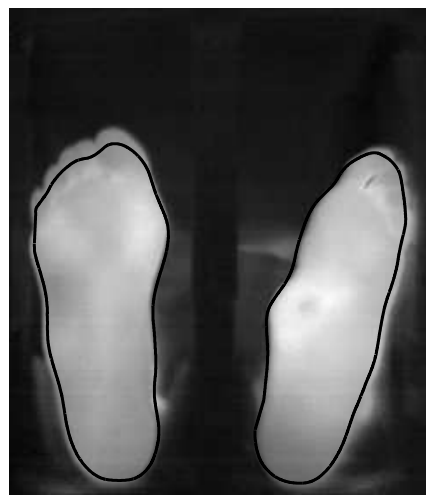

(a) Contour Ill-positioned

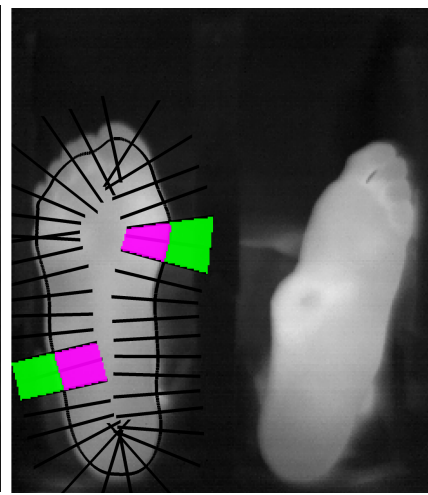

(b) Line and Polygonal Profile

Figure 5.5: (a) Examples of ill-positioned feet contours, mapping directly from colour image segmentation. (b) Illustration of the perpendicular line profiles of the foot contour, which defined the polygonal mask in and out of the foot contour. Note the left panel displays longer profile lines (length $=51$ pixels) and larger polygonal window for illustrative purposes. 
was located at the toes area. This is explained by the fact that when the patients' feet were well positioned onto the foot supporters underneath the heels, these heels were near the projective plane. Examples of the misalignments can be found in Fig. 5.5a.

A patient-specific fine registration was needed to correct this possible misalignment. A rigid transformation consisting of translation, rotation and vertical scaling was assumed to suffice. Four parameters are needed to define this transformation. Horizontal scaling was not needed as the deviations between foot soles and projective plane were mainly slants in the longitudinal direction of the foot soles.

To optimise the four parameters, a quantitative criterion was needed. We selected a criterion based on the observation that the statistics of the temperatures in a region inside the foot will differ the most when compared with statistics of a region entirely outside the foot. We defined pairs of small regions positioned at both sides of the hypothesised foot contour. The difference between histograms of the temperatures in these opposite regions should be maximised. By the aggregation of a measure of this difference, calculated along different points on the hypothesised foot contour, a global optimisation criterion was found. Although parts of the foot, such as the toes, had low contrast against the background, the contour points at these regions had low importance in driving the registration optimisation.

The regions were defined at a set of foot contour points $\left\{\left(x_{c}, y_{c}\right) \mid c=1, \cdots\right\}$ resulting from the colour segmentation. A polygonal perpendicular was placed at each contour point as shown in Fig. 5.5b. The polygon was split into two parts, $R_{i n}\left(x_{c}, y_{c}\right)$ and $R_{\text {out }}\left(x_{c}, y_{c}\right)$, representing the parts inside and outside of the foot area. The measure of difference between $R_{\text {in }}$ and $R_{\text {out }}$ at each point $\left(x_{c}, y_{c}\right)$ was defined as the $\chi^{2}$ distance between the histograms $h^{i n}$ and $h^{\text {out }}$ in the polygons:

$$
\chi^{2}\left(x_{c}, y_{c}\right)=\frac{1}{2} \sum_{i} \frac{\left(h^{\text {in }}(i)-h^{\text {out }}(i)\right)^{2}}{h^{\text {in }}(i)+h^{\text {out }}(i)}
$$

Aggregation of all these distances along the contour yields:

$$
D=\sum_{c} \chi^{2}\left(x_{c}, y_{c}\right)
$$

Fine registration was done by changing the four transformation parameters so as to minimise $D$. The optimisation was implemented with the optimisation toolbox in Mat lab with the sequential quadratic programming algorithm 
searching for the local maximum of $D$ within a bounded region of four parameters.

\subsubsection{Asymmetric Analysis of Diabetic Foot Complications}

left and right feetThe temperature difference of corresponding points on the left and right feet is an indicator for diabetic foot complications $[5,6]$. However, the two feet in the thermal images were hardly at symmetric positions in the image, and they often showed asymmetric deformations due to DM or due to amputations. Therefore, the left and right feet needed to be registered with each other.

The goal of the left and right feet registration was to find the optimal transformation $\mathbf{T}:(x, y) \mapsto\left(x^{\prime}, y^{\prime}\right)$, which maps any point $(x, y)$ in the segmented left foot onto the corresponding point $\left(x^{\prime}, y^{\prime}\right)$ in the segmented right foot. Due to local foot deformations (caused by DM), amputations, and projection distortions, a rigid left to right foot registration did not suffice, and a non-rigid registration was needed. We employed a landmark-based deformation model based on B-splines [114,115]. This model consists of an orthogonal $n_{x} \times n_{y}$ mesh that covers the image plane. The shifts of the mesh points form the parameters $\Phi$ of the registration. The shifts of pixels between grid points are obtained by the B-spline interpolation. To find $\boldsymbol{\Phi}$, a number of corresponding landmarks on the left and right feet are needed. Due to deformities and amputation, points at the lower half (near the heel) are more stable than the one near the toes. The algorithm to obtain these corresponding points is as follows:

Algorithm: Selection of corresponding points in the left and right feet

- Inputs: Contours $\left(x_{n}^{l}, y_{n}^{l}\right)$ and $\left(x_{n}^{r}, y_{n}^{r}\right)$ with $n=1, \cdots$. These are sequences of $2 \mathrm{D}$ coordinates in the thermal image denoting the boundaries of the left and right foot regions in the thermal image. left and right feet

- Selection in left foot and in the right foot: (See Fig. 5.6)

- Determine the centroid $\left(x_{c}^{l}, y_{c}^{l}\right)$ of the contour points.

- Determine the midline of the lower half of the foot: a) Determine for each horizontal line segments that connects the left boundary point to a right boundary point, and that is below the centroid, the midpoint. b) Determine the least squares linear fit of these midpoints.

- Rotate the contour around the centroid such that the midline stands vertical.

- Shift the contour vertical such that the lowest point (the heel) is at the x-axis.

- Define horizontal lines on either foot with normalisation with respect to the foot lengths. The vertical spacing of these lines below the centroid is smaller than above the centroid. 
- The intersection points of these horizontal lines with the foot contours are the landmarks (together with the heel points). Detect whether a part is missing on either foot. If so, delete these points from the list.

To find the best parameters $\Phi$, the sum of squared differences between transformed left landmarks and the corresponding right landmarks was minimized with a steepest descent algorithm. The gradient vector, which is needed for this optimisation, was obtained numerically.

After estimation of the parameters of registration, the associated geometric transformation was applied to the left foot to make the pixels to correspond with the pixels on the right foot. The likelihood of a foot complication of a spot was calculated simply by subtraction of temperatures of corresponding pixels. The threshold we used for complications detection is $2.2^{\circ} \mathrm{C}[5,6]$. This criterion is the only one that has been clinically validated for determining diabetic foot complications with temperature measurements.

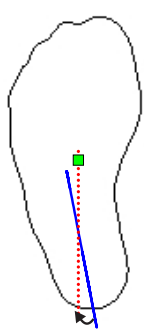

(a) Right Foot

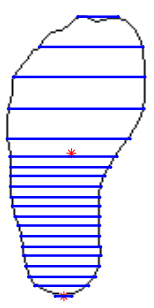

(b) Right Foot

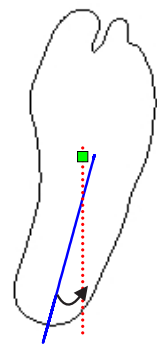

(c) Left Foot

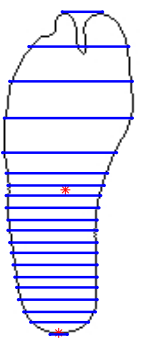

(d) Left Foot

Figure 5.6: Selection of corresponding landmarks in the left and right foot contours for left/right foot registration. (a) and (c): The midlines of the lower halves of the foot regions were used to align the feet vertically. (b) and (d): Horizontal line scanning with two different step sizes.

To evaluate the registration results, 4 corresponding regions on the left and the right feet of each patient were manually labelled. These regions were located near the border of the foot at top, bottom, medial, and lateral positions. If any region of either foot had been amputated, no region was selected. Examples of the annotation can be found in Fig. 5.9. Comparison between the labeled regions on the right foot and the regions on the registered left foot was done to obtain a rough measure of the registration quality. This evaluation is limited by the subjectiveness of the manual labelling. 


\subsection{Results and Discussion}

\subsubsection{Feet Segmentation Results in colour Images}

Initially, six colour spaces were considered: RGB, normalised RGB (rgb), RGBratio, HSV, CIE $\mathrm{L}^{\star} \mathrm{a}^{\star} \mathrm{b}^{\star}$ and $\mathrm{YCbCr}$. All have three colour channels. For two of them, CIE $\mathrm{L}^{\star} \mathrm{a}^{\star} \mathrm{b}^{\star}$ and $\mathrm{YCbCr}$, it might make sense to skip the luminance channel yielding the $a^{\star} b^{\star}$ and $\mathrm{CbCr}$ spaces. This left us with eight possible spaces. A space can be processed by the K-means, the EM-LDC, or by EM-QDC. The considered presets of $K$ were 2, 3, and 4 . The values were determined based on the visual inspection of the number of objects (i.e. feet, black foot side supporting bars, black cloth, aluminium bars and the white hospital cloth) in the acquired RGB images. Thus, in full, 72 combinations of spaces, algorithms, and presets could possibly lead to a viable method.

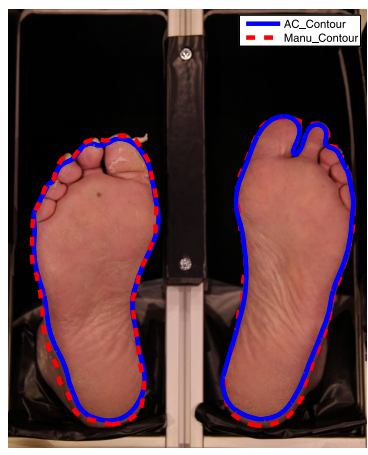

(a) Example 1

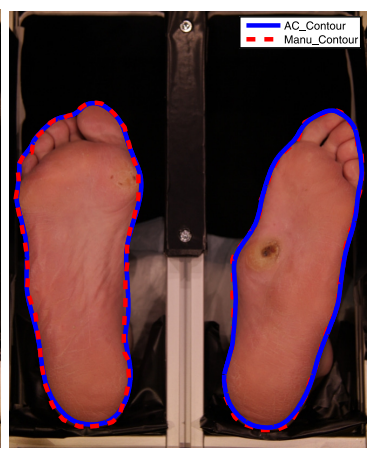

(b) Example 2

Figure 5.7: Examples of foot segmentation done with EM-QDC using 3 colour channels in CIE $\mathrm{L}^{\star} \mathrm{a}^{\star} \mathrm{b}^{\star}$ (blue solid line), and the manual segmentation (red dashed line).

A pre-selection of combinations/methods was made based on the following criterions:

- Visual inspection of the segmentation results. If a method clearly showed inferior results, it was deleted from the list.

- Near resemblance of performance. All methods based on the RGB space and on the $\mathrm{YCbCr}$ space had nearly identical performance. This might 


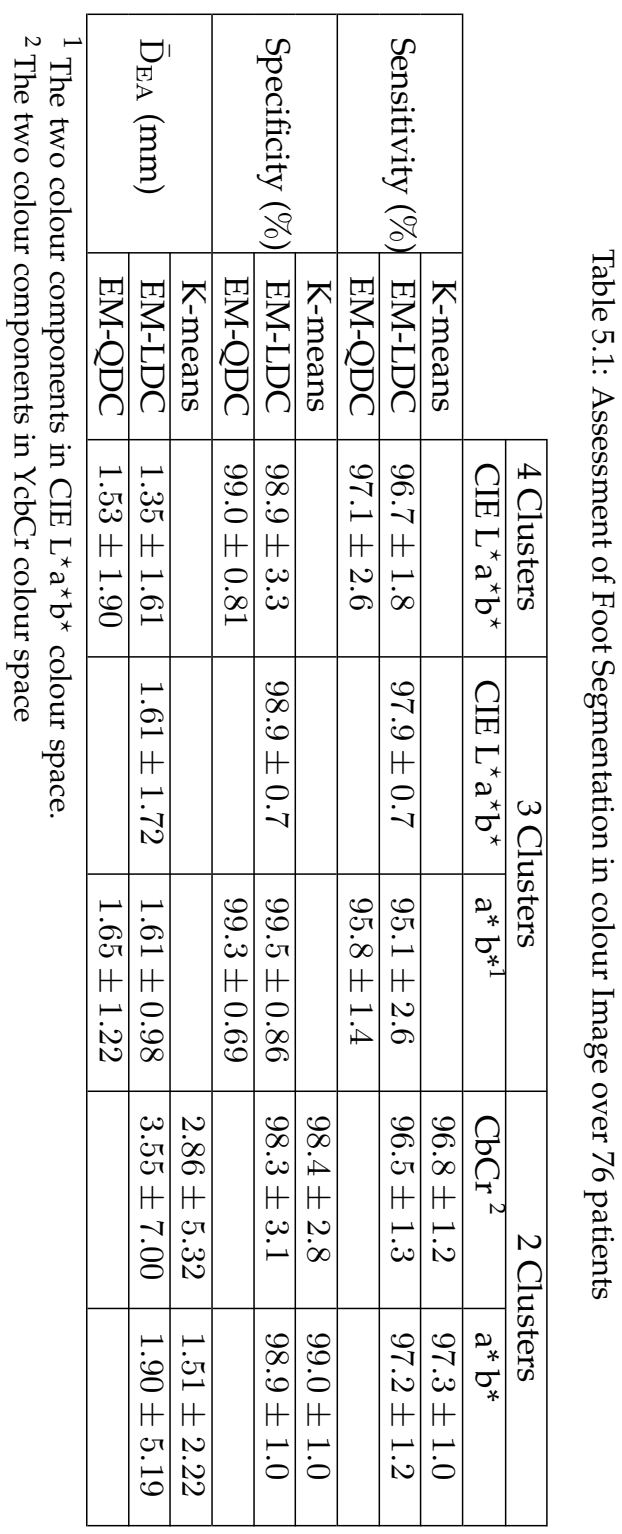


be explained by the way that $\mathrm{YCbCr}$ encodes RGB. Anyway, this resemblance motivated us to delete the $\mathrm{YCbCr}$ based methods.

- Assessed performance measures. If the sensitivity, averaged over the population of patients, was less than $95 \%$, the method was deleted from the list. Likewise, if the specificity was less than $95 \%$, or the mean distance error $\bar{D}_{E A}$ (averaged over the population) was larger than $10 \mathrm{~mm}$, the method was also excluded from consideration.

- Instability. If the standard deviation of the sensitivity or specificity, calculated over the population, was larger than 15\%, the method was crossed out for it was not capable of segmenting the feet with a stable performance.

Only 9 methods, from the 72, survived these elimination rules. The quantitative assessment of the remaining methods can be found in Table 5.1. The difference between the performance of these 9 methods are not very statistically significant except for $\mathrm{a}^{\star} \mathrm{b}^{\star}$ with EM-LDC or EM-QDC and $K=3$. This method presented relatively lower mean sensitivities, just above $95 \%$, but the mean specificities were the highest. This indicates an increased risk for assigning the foot area to the background. $\mathrm{CbCr}$ with all the methods and $K=2$ obtained relatively higher $\bar{D}_{E A}$ and relatively lower sensitivity. Thus, the segmentations with $\mathrm{CbCr}$ for 2 clusters, and those with $\mathrm{a}^{\star} \mathrm{b}^{\star}$ for 3 clusters for all methods were excluded as well.

To conclude, the foot segmentation from the colour images can be achieved in CIE $\mathrm{L}^{\star} \mathrm{a}^{\star} \mathrm{b}^{\star}$, either using all the 3 colour channels with EM-QDC for 4 clusters, or EM-LDC for 3 or 4 clusters, or using only the 2 colour components through K-means, EM-LDC, EM-QDC for 2 clusters. Two examples of the segmentation results are illustrated in Fig. 5.7. The sensitivities and specificities of these combinations were all around $98 \% \pm 1 \%$ and $99 \% \pm 1 \%$, respectively, and $\bar{D}_{E A}=2 \pm 2 \mathrm{~mm}$. In the following sections, results are presented based on the foot segmentations obtained using all the 3 colour channels in CIE $L^{\star} a^{\star} b^{\star}$ with EM-LDC for 3 clusters.

\subsubsection{Registration Optimisation in the Thermal Images}

The parameters of the fine registrations are the shift $\left(x_{t}, y_{t}\right)$, the rotation angle $r$, and the vertical scale $s$. Since the pivot of the rotation was near the heel, the foot contour was not rotated around its centroid point, but around the heel 


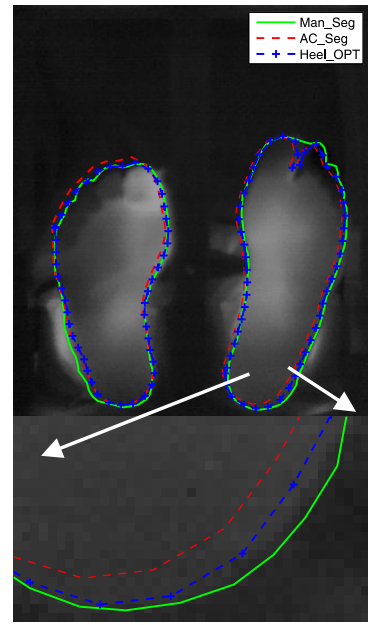

(a) Example 1

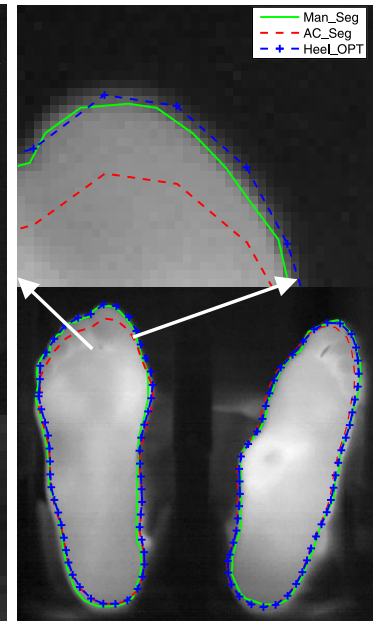

(b) Example 2

Figure 5.8: Examples of results from optimised registration. The green solid lines represent the foot contours obtained from manual segmentation done by experienced clinicians. The red dashed lines correspond to the foot contours obtained from projective transformation of the contours from the colour images. The blue dashed lines come from the optimised registration.

point (the furthest point on the foot contour when the foot was rotated to stand vertically).

The length of the perpendicular profile line (shown in 5.5b), which is equivalent to the window size, was set to 11 pixels with 5 pixels inside, and 5 pixels outside the foot region. To bound the search area, the ranges of the parameters were limited; for shift: $x_{t}, y_{t} \in[-20,20]$ (pixel), for rotation: $r \in[-3,3]$ (degree), and for vertical scaling: $s \in[0.95,1.05]$. Examples of the optimisation results can be found in Fig. 5.8.

Compared to manual segmentation in the thermal images, the sensitivity and specificity of feet segmentation in the thermal images with transformation parameter optimisation, before fine registration, are $96.0 \% \pm 2.0 \%$ and $98.1 \% \pm$ $0.7 \%$ with $D_{E A}=2.7 \pm 1.0 \mathrm{~mm}$; and after fine registration: $97.9 \% \pm 1.1 \%$ and $98.3 \% \pm 0.5 \%$ with $\bar{D}_{E A}=1.9 \pm 0.6 \mathrm{~mm}$. 


\subsubsection{Left and Right Foot Registration and Detection of Dia- betic Foot Complications}

For each patient, the right foot was chosen as the static object, and the left foot was mapped on the right foot. Examples of the registration between left and right feet and the detected complications are given in Fig. 5.9.

To get a quantitative assessment of the registration results, a comparison between manually association of some regions on the right and left foot and automated association (by our right/left registration algorithm) was performed: the sensitivity and specificity are $85 \% \pm 1 \%$ and $98.4 \% \pm 0.4 \%$, respectively, and $\bar{D}_{E A}=4 \pm 1 \mathrm{~mm}$. The error may come partly from the subjectiveness of the manual association, and partly also from the asymmetric deformations of the feet (which can locally make the association difficult, or even impossible). Taking this into account, the registration results are considered to be accurate.

Taking $2.2^{\circ} \mathrm{C}$ as cut-off point for risk identification, all $3 \mathrm{Charcot}$ foots and 35 out of 37 diabetic foot ulcers were successfully detected (95\%). The 2 missed ulcers were small $\left(\sim 5 \times 5 \mathrm{~mm}^{2}\right)$, one of which was only found after debridement of abundant callus.

\subsubsection{Limitations of the Study}

A limitation of the proposed asymmetric analysis was that it could only detect diabetic foot complications by comparing the two feet of the patient. This means that when one foot is amputated, any complication in the other foot can not be detected. Additionally, in case that both feet have similar complications in corresponding regions, the complications will also be missed. Besides, in finding the asymmetry of between left and right feet, registration was always done from left and right feet. This registration itself may introduce artificial asymmetry, which has not been investigated in this article.

Another limitation of the study is that we did not have a 'gold standard' to evaluate the segmentation and registration of the feet. We used manuallysegmented references that were labeled by one clinical investigator. This may be subject to some bias since another observer may come to different observations. However, we expect that this inter-observer variability will not significantly affect the found sensitivity and specificity. 


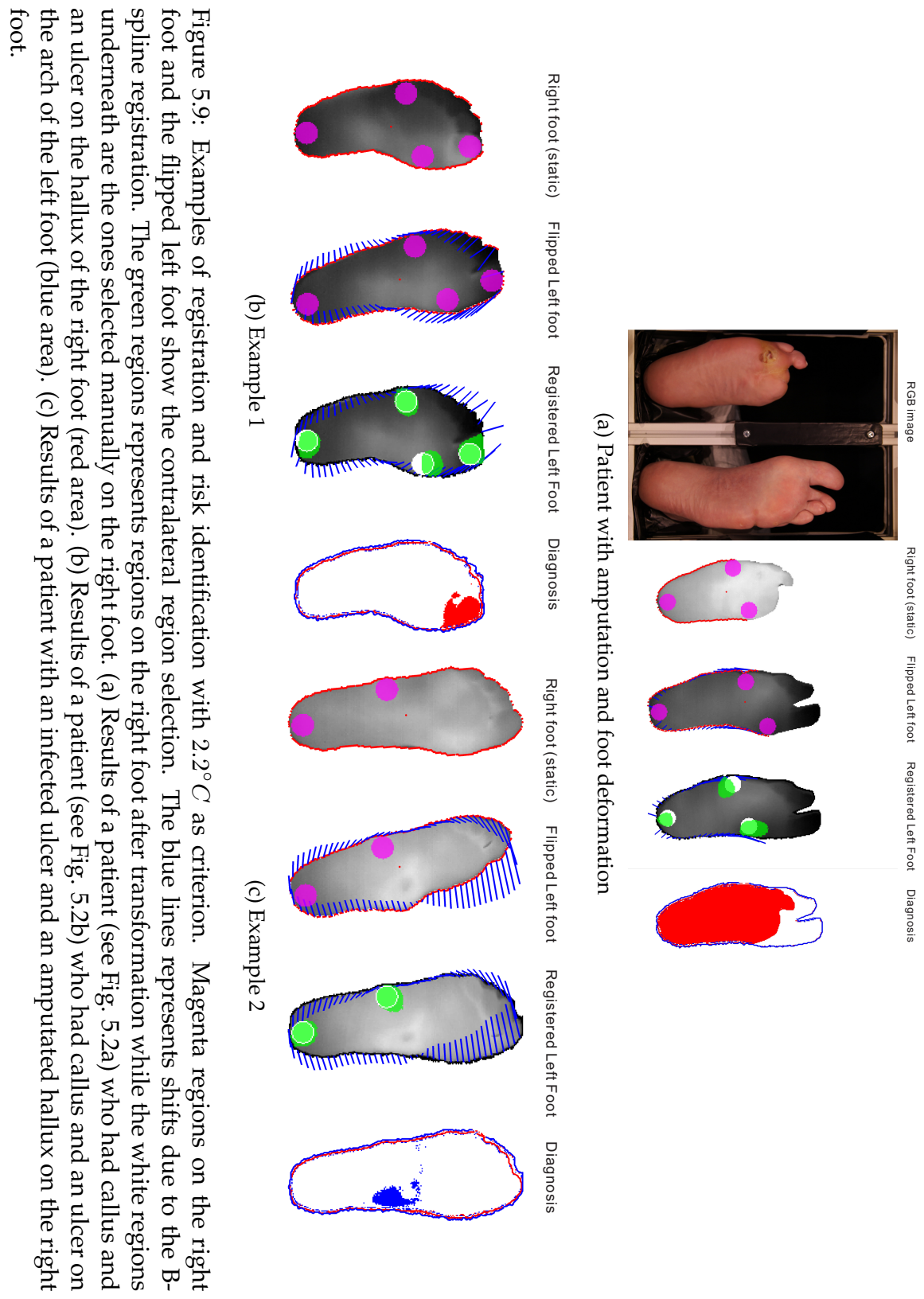




\subsection{Conclusion}

In this contribution, an experimental setup with an IR camera system and an RGB camera was built. With the data collected from diabetic patients with foot complications, or patients who are at high risk of foot complications, a methodology was proposed for automated detection of these foot complications with the acquired thermal images. The method is based on a simple asymmetric analysis combined with foot segmentation from color images and non-rigid registration between left and right foot.

Foot segmentation with unsupervised machine learning in different color spaces was investigated in Section 5.4.2. The foot segmentation in CIE $\mathrm{L}^{\star} \mathrm{a}^{\star} \mathrm{b}^{\star}$ color space achieved a sensitivity and specificity of $98 \% \pm 1 \%$ and $99 \% \pm 1 \%$, respectively. With registration optimization to map the foot segments onto the thermal image, the final sensitivity and specificity of the foot segmentation in the thermal images were $97.9 \% \pm 1.1 \%$ and $98.3 \% \pm 0.5 \%$, respectively. The registration between the left and right foot, based on foot contours, presented fairly good results regardless of the shapes, sizes, poses or positions of the two feet. In this chapter, we provide the proof-of-principle that the thermal images acquired through our experimental setup can detect diabetic foot complications.

These promising outcomes of thermal image analysis of the foot may prove to be promising in the early detection of foot complications in patients with DM and patients who are at high risk for these complications. Future research objectives are: $a$ ) Both the left and right feet may be restored to a general and unbiased foot contour template to reduce the asymmetry in the foot registration. b) Thermal image analysis of the ipsilateral foot to detect diabetic foot complications without the need to image the contralateral foot. c) Combining the thermography technology with other modalities, such as photometric stereo imaging [83] and multispectral imaging [40], in the experimental setup to predict the development or healing of diabetic foot complications. d) Comparing the effectiveness and efficiency of predicting diabetic foot complications using different modalities; $e$ ) Developing and investigating an intelligent telemedicine system with the most cost-effective modality or modalities to monitor diabetic foot status in daily clinical practice. 



\section{Photometric Stereo Imaging to Reconstruct the 3D Surface of the Plantar Foot of Diabetic Patients: A Feasibility Study on Detection of Diabetic Foot Complications}

\subsection{Abstract}

Photometric Stereo Imaging is capable of reconstructing and presenting details of both the skin geometric and radiometric changes, which makes it a potential modality for an intelligent telemedicine system for frequent screening and risk assessment of diabetic foot complications. In this chapter, we describe the development and investigation of a PSI system with eight illuminators for scanning the foot soles of patients with Diabetes Mellitus. A feasibility study was conducted to apply the 3D surface reconstruction on plantar foot from diabetic patients using photometric stereo imaging. The 3D curvature features would be extracted and be used as image features for foot diagnostic segmentation to distinguish between the skin with and without any diabetic foot complications. When the patient feet remain static during measurement, the Root Mean Square Error of the height map reconstruction, based on our PSI system and reconstruction method, was $0.5 \mathrm{~mm}$. By combing the recovered surface radiometric property, and the 3D curvature features extracted from the recovered surface normal as input features to Expectation-Maximisation clustering for detecting diabetic foot complications, the sensitivity and specificity over 34 patients were $81.4 \%$ and $82.3 \%$, respectively, comparing with the clinical live assessment. These outputs indicated PSI as potential technique for detecting the diabetic foot complications for further study. Additional research and investment is worthwhile, which unfortunately will not be included in this thesis. 
This chapter will be published in a slightly modified version as "C.Liu, J. J. van Netten, J. G. van Baal, S. A. Bus, and F. van der Heijden, Photometric Stereo Imaging to Reconstruct the 3D Surface of the Plantar Foot of Diabetic Patients: A Feasibility Study on Detection of Diabetic Foot Complications"

\subsection{Introduction}

Diabetes Mellitus (DM) is on the rise in prevalence and significance worldwide [2]. Diabetic foot ulceration is one of the major complications for patients with DM. Approximately $15 \%-25 \%$ of diabetic patients will eventually develop foot ulcers [3]. These ulcers provide an avenue for infection, and may cause progressive tissue necrosis. If not adequately treated, they may lead to an increased risk of amputation and mortality [7]. Early risk identification and subsequent treatment of diabetic foot complications (e.g. ulceration, inflammation, callus formation and blisters), plays a pivotal role in amputation prevention and diabetes care. However, it is impractical and expensive to have frequent risk assessment by health care specialists. Examination by patients themselves has its limitations due to various health impairments caused by DM. Thus, any innovation that is noninvasive, non interactive and easy to use, for automatic assessment and detection of diabetic foot complications, may be beneficial for diabetic foot care. The ultimate objective of our project is to develop an intelligent telemedicine monitoring system, that can be deployed at the patients' homes or at health centres, for frequent foot assessment in order to detect diabetic foot complications in due course.

\subsubsection{Related work}

\section{Telemedicine system using digital photography}

Telemedicine systems based on digital photography have been built and tested for frequent foot risk assessment [19,32]. However, some pre-signs of diabetic foot ulceration are difficult to assess using digital photography [32]. Furthermore, such systems are not yet capable of automatic assessment of diabetic foot complications. This limits their application and implementation.

\section{D Scanning Technologies}

3D shape acquisition and reconstruction is a beneficial but also a challenging problem in many fields of engineering and life science. It may be useful for 
revealing the local 3D surface deformation of the foot sole skin surface, which then provides information about changes in the surface texture. Such changes are either caused by geometrical variation of the surface (e.g. papillary lines, or pre-signs of ulceration such as fissures, abundant callus) or by radiometrical variations (e.g. necrosis, infection related redness, or an ulcer).

Numerous 3D scanning technologies exist. They generally fall into three categories, which are multi-view imaging, photometric stereo imaging (PSI) and tactile imaging. Multi-view imaging techniques often require elaborate setups [75], and thy focus more on the global 3D shape reconstruction, thereby failing to capture high-frequency structure details [76]. Tactile imaging systems measure the local pressure on the object surface and they translate the measured pressure to a 3D surface [77]. The pressure on the foot skin is fundamental for tactile imaging, but may cause the transmission of pathological organisms in our application. PSI, on the other hand, is a modest and noncontact technology, which achieves the 3D surface reconstruction based on an image set taken from one fixed viewpoint under three or more different illumination conditions $[78,79]$. In PSI, low spatial frequency components of measured gradients are rejected and 3D surfaces are derived based on higher spatial frequency components, which can capture the local surface details. As such, PSI shows greater potential for future telemedicine applications and it is the focus of this chapter.

In this chapter, we first describe the development of a PSI system with eight illuminations for diabetic foot sole scanning. And the feasibility of the PSI system in detecting diabetic foot complications was investigated in a group of patients at high risk for diabetic foot ulceration. Currently, the study was oriented to show the capability of the PSI in distinguishing between skin with diabetic foot complications and healthy skin.

\subsubsection{Outline of this chapter}

The fundamental theory of PSI reconstruction was introduced in Section 6.3. Section 6.4 describes our experimental setup, the measurements and patient recruitment. Next, the image processing for 3D surface reconstruction and the detection of diabetic foot complications are described in Section 6.5. Results and discussion follow in Section 6.6. Finally, conclusions and recommendations for further work are given in Section 6.7. 


\subsection{Photometric Stereo Imaging}

The fundamental theory of PSI is based on two assumptions [78,79], which are (a) all illumination sources are at a large distance and they can be regarded as point sources at known positions. This provides parallel incident light with known illumination directions. (b) The object surface is Lambertian, i.e. the surface remission is an isotropic scattering and it follows a Lambertian reflectance model, without shadows or specular reflections.

A given image point $(u, v)$ corresponds in 3D to an unknown depth $z=$ $f(u, v)$. Here, $(u, v)$ are the pixel coordinates. Its 3D position in world coordinate system can be derived as $\mathbf{p}=\mathbf{P}^{-1}[u, v, 1]^{T}$, where $\mathbf{P}$ denotes the camera's perspective transformation.

Suppose the 3D position $\mathbf{p}$ on the object surface has a surface norm $\mathbf{n}$ and a surface albedo $\rho$. Under the PSI assumption, the pixel brightness in the acquired image $\left(I_{i}(u, v)\right)$ under illumination source $\mathbf{l}_{i}(i=1 \cdots K, K$ is the number of illumination sources) can be described by the Bi-direction Reflectance Function (BRDF), as follows:

$$
I_{i}(u, v)=A_{i}(u, v) \rho(u, v) \mathbf{l}_{i}(u, v) \cdot \mathbf{n}(u, v)
$$

where $A_{i}(u, v)$ is a response factor, including the camera sensor sensitivity and the illumination strength attenuation.

Defining $m_{i}(u, v) \equiv I_{i}(u, v) / A(u, v)$, for a PSI system with $K$ illumination sources, we may stack the $\mathrm{K}$ equations to a linear system:

$$
\mathbf{M}(u, v)=\rho(u, v) \mathbf{L}(u, v) \mathbf{n}(u, v)
$$

The above linear system of equations can be solved if the illumination sources $\mathbf{L}(u, v)$ is nonsingular. This is equivalent to requiring that the illuminations sources and the objects do not lie in the same plane. The more illuminators involved, the more accurate and robust the recovery result may be. The surface normal and the surface albedo can be estimated according to the least square estimation as follows $[78,79]$ :

$$
\begin{aligned}
\rho(u, v) & =\left|\left[\mathbf{L}^{T}(u, v) \mathbf{L}^{-1}(u, v)\right] \mathbf{L}^{T}(u, v) \mathbf{M}(u, v)\right| \\
\mathbf{n}(u, v) & =\frac{1}{\rho(u, v)}\left[\mathbf{L}^{T}(u, v) \mathbf{L}^{-1}(u, v)\right] \mathbf{L}^{T}(u, v) \mathbf{M}(u, v)
\end{aligned}
$$

For RGB images, the estimation by Eq. 6.3 can be done for each color channel, and the surface normal can be achieved as an average as $\mathbf{n}(u, v)=$ $\left[\mathbf{n}_{r}(u, v)+\mathbf{n}_{g}(u, v)+\mathbf{n}_{b}(u, v)\right] / 3$. 
The directional gradients can be obtained by scaling the directional normal to its surface:

$$
\begin{aligned}
{\left[\begin{array}{lll}
-\frac{\partial f(u, v)}{\partial u} & -\frac{\partial f(u, v)}{\partial v} & 1
\end{array}\right] } & =\frac{\mathbf{n}(u, v)}{n_{z}(u, v)} \\
& =\left[\begin{array}{lll}
-\frac{n_{u}(u, v)}{n_{z}(u, v)} & -\frac{n_{v}(u, v)}{n_{z}(u, v)} & 1
\end{array}\right]
\end{aligned}
$$

\subsection{Materials and Measurement}

An experimental setup, consisting of one commercial digital RGB camera, the Canon EOS 40D, and eight power LED light sources [83], has been developed for measurements on patients as shown in Figure 6.1. The digital camera was placed at a distance of $860 \mathrm{~mm}$ from the measurement plane, covering a field of view $420 \times 280 \mathrm{~mm}^{2}$ with resolution $3888 \times 2592$ pixels (pixel size $\approx 0.1 \mathrm{~mm}$ ). The eight illumination sources were mounted on a circle $(\phi \approx 565 \mathrm{~mm})$ with equally spaced tilt angles. The distance between the target imaging plane and the illumination plane was $480 \mathrm{~mm}$. As such, the slant angles for each illumination sources were about the same, $\approx 30^{\circ}$, satisfying the requirement for photometric stereo imaging system design [80].

Patients were recruited from the multidisciplinary diabetic foot clinic of the Hospital Group Twente, Almelo, the Netherlands. The patients included in this study were all diagnosed with DM and showed diabetic foot complications, e.g. callus, blisters, redness, ulceration, or had a history of ulceration. The average age of the 34 included patients, 25 males and 9 females, was 65 years $(\mathrm{SD}=13.8)$.

Measurements started with placing patients' feet on the foot support of the experimental setup (Fig.6.1e). To help to position the patients' feet and restrict the movements of the feet during measurement, three sidelong supporting bars were mounted. The patients were allowed to lean their foot on the bars during measurement. The positioning of the patients' feet was adjust according to the top surface of the supporting bars to make sure all the patients' feet almost at the same plane. This adjustment was done with a help of the clinical experimenter by visual check. A hospital cloth was placed over the legs of the patients for hygiene reasons and one piece of black cloth was draped over the shield of the setup to block external light entering the setup and to provide a homogeneous background. During the measurement, the eight illumination sources (Fig.6.1c) were turned on one by one. For each of the illuminators, one image was taken. As such, for each measurement, an image set of eight images 


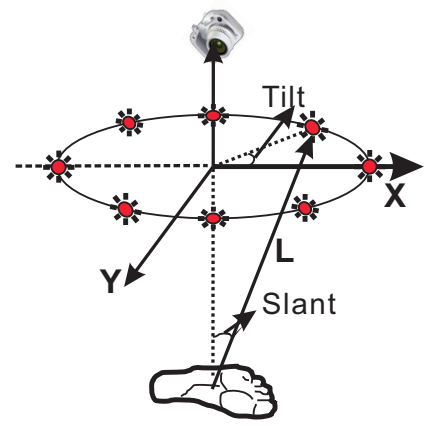

(a)

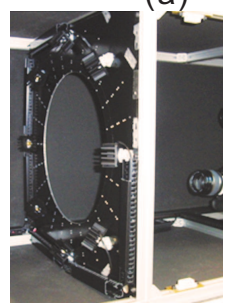

(c)

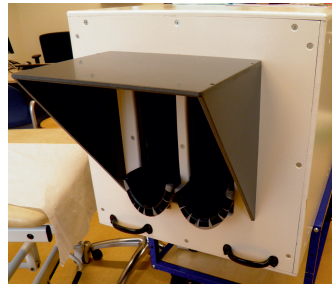

(d)

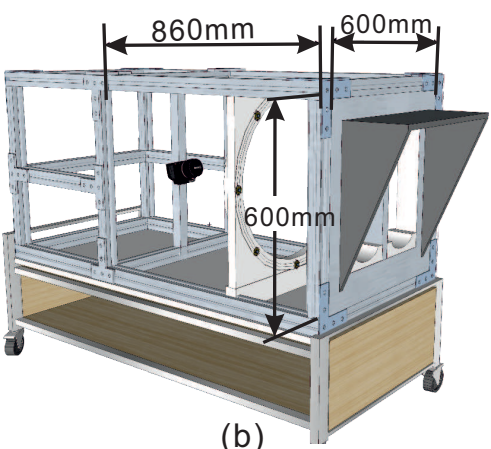

(b)

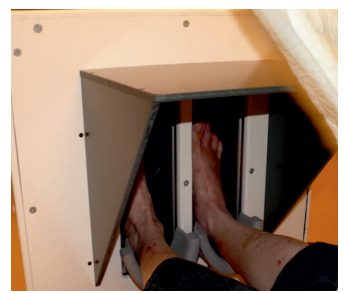

(e)

Figure 6.1: The experimental setup with eight illuminations. (a) The schematic of PSI with eight illuminations (The thermal camera and spectral camera array were removed from the schematic for illustration purpose); (b) The sketch of the experimental setup for patient measurements; (c) The illumination plate and the RGB camera; (d) The appearance of the experimental setup; (e) Positioning of patient's feet during the measurement.

was acquired by the same digital camera with 8 different illumination sources. The total measurement takes around 90 seconds, during which the patients were requested to keep their foot as stable as possible to avoid any movement.

For each patient, a live assessment form of the plantar surface of both feet was completed by a wound care specialist. This live assessment was used as a reference for the validation of the automatic detection. Examples of the acquired images under different illuminators are given in Fig. 6.2.

\subsection{Method}

Preceded with system and light calibration (Section 6.5.1), first, patient specific PSI processing was done, and next to which was diabetic foot complica- 


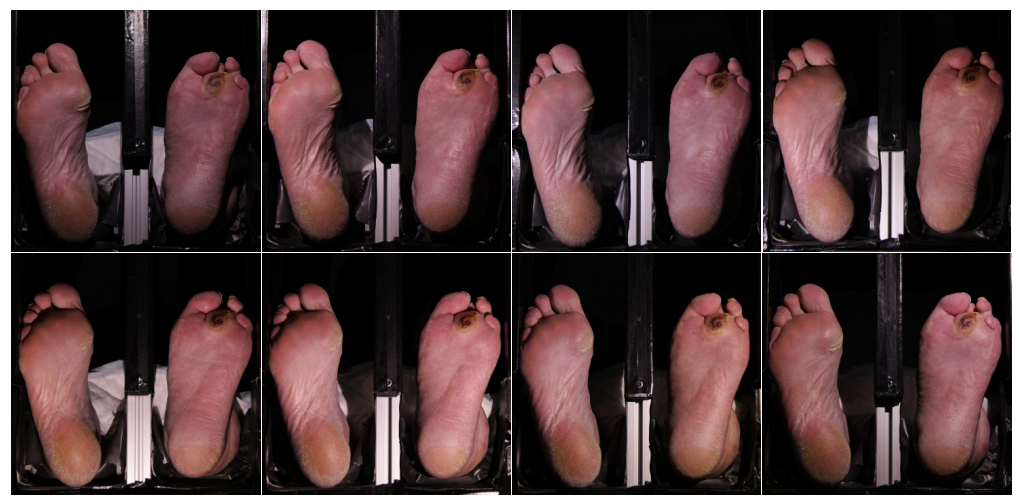

Figure 6.2: The acquired images set for one patient under eight illuminators. The images are cropped for illustrative purpose.

tions detection. This was achieved in three steps: image preprocessing (Section 6.5.1), PSI reconstruction (Section 6.5.2) and the detection of foot complications (Section 6.5.3). All the image processing and pattern classification in this study was performed on Matlab R2013b, with an additional toolbox PRtools5 [86] in 64 bits Windows.

\subsubsection{Calibration and Image Pre-processing}

Before measurements on patients, the imaging system was calibrated using the camera calibration toolbox [119] to obtain the camera perspective transformation matrix $\mathbf{P}$.

To calibrate the light distribution over the field of view (FOV), three calibration objects, shown in Fig. 6.3, were built and imaged, with known surface geometry (known surface normal). The surface of the three calibration objects were covered with standard Grey Card (18\% neutral grey, Fotowand Technic, Sudwalde, Germany), which created objects with known surface albedo $\rho_{\text {gray }}=0.18$.

For each pixel in the FOV, by stacking all the image intensities of the three calibration objects under the same illuminator, a linear equation system based on Eq. 6.1 can been built as

$$
\mathbf{I}_{i}^{\text {gray }}(u, v)=\rho_{\text {gray }} A_{i}(u, v) \mathbf{l}_{i}(u, v) \mathbf{n}(u, v)
$$


which can be solved to obtain the illumination distribution $\mathbf{l}_{i}(u, v)$ and an estimation of the response factor $A_{i}(u, v)$, which are needed for solving Eq. 6.2. This light calibration needs to be done off-line once for the same setup.

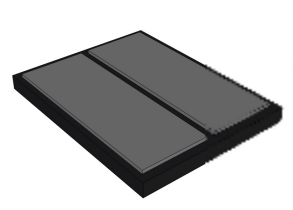

(a)

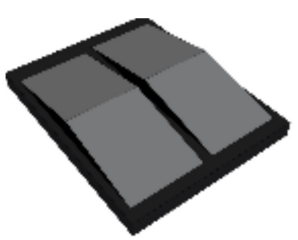

(b)

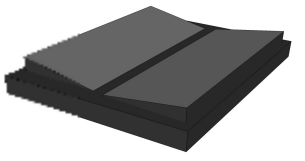

(c)

Figure 6.3: Three calibration objects with different surfaces geometries

Foot segmentation was preceded by PSI reconstruction with unsupervised machine learning technology for each set of the input images. Details about this have been addressed in [120]. In this way, the PSI reconstruction would be focused on the foot area only to avoid the influence of the background.

\subsubsection{Surface Reconstruction}

Though the Lambertian assumptions can accurately describe the diffuse surface reflection, it may sometimes oversimplify the reflectance of real world objects, especially when the objects exhibit shiny highlights or when are subject to self-occluding shadows. Specularity and shadows are usually difficult to eliminate in PSI reconstruction.

Our method for PSI reconstruction combined the one-specularity-twoshadow assumption [81] and the simple decision criterion proposed by Reshmeier et al. [121]. In the pixel-wise PSI reconstruction, the $\mathrm{K}(\mathrm{K}=8)$ grey-level image intensities were ranked in descending order. The assumption that at most one specular reflection and two shadows are present among the multiple images has been proven to work well with most objects [81]. In view of this, the highest and the last two lowest image intensities, together with their corresponding illumination sources, were always discarded. As such, five of the measured intensities were taken into account for the reconstruction to rule out the influence of shadows and specular reflection.

As simple as the criterion is, there might be a risk to lose information in the highest or the two lowest values that are not associated with specular reflection or shadows. In our system, we still have five, two more than three, images, which may provide redundant information as extra equations in Eq. 6.2, to 
improve the accuracy and robustness of the recovery presented in Eq. 6.3. All these aspects make our method easy and robust to implement.

To obtain the height information from the recovered surface normal, the common method based on Fourier basis was implemented [122]. In this method, the surface gradients $[\partial f(u, v) / \partial u, \partial f(u, v) / \partial v]$ were projected onto a set of Fourier basis functions to do the integration in the frequency domain, which enhanced the integrability and the tolerance to noises.

Currently, it is hard to obtain the ground truth for the foot sole of a patient. Therefore, for validation two wooden half spheres $(\phi=20 \mathrm{~mm})$ attached to the cover of a grey hard board were used. And their 3D surfaces were reconstructed. This validation targets were also used to capture the influence of possible movement of the patients.

\subsubsection{Detection of Diabetic Foot Complication}

Surface curvature can be an important 3D feature for revealing the surface intrinsic properties. For each pixel, the Gaussian curvature $(K)$, the mean curvature $(H)$ and the scalar curvature $(C)$ can be calculated as follows [123]:

$$
\begin{aligned}
K & =\frac{f_{u u} f_{v v}-f_{u v}^{2}}{1+f_{u}^{2}+f_{v}^{2}} \\
H & =\frac{\left(1+f_{u}^{2}\right) f_{v v}+\left(1+f_{v}^{2}\right) f_{u u}-2 f_{u} f_{v} f_{u v}}{\left(1+f_{u}^{2}+f_{v}^{2}\right)^{3 / 2}} \\
k_{1}, k_{2} & =H \pm \sqrt{H^{2}-K} ; \quad C=\sqrt{k_{1}^{2}+k_{2}^{2}}
\end{aligned}
$$

where $f_{u}, f_{v}$ and $f_{u u}, f_{v v}, f_{u v}$ are the first and second order partial derivatives of the depth $f(u, v)$. An example of the scalar curvature $(C)$ can be found as Fig. 6.7b.

The recovered surface albedo and the surface curvature were used as input features for the Expectation-Maximisation (EM) clustering, aiming at the detection of diabetic foot complications. The EM clustering is one of the most popular techniques in unsupervised machine learning [86]. A probabilistic model $f_{e m}$ is used to estimate the joint probability distributions $p\left(\mathbf{x}, k_{l} \mid f_{e m}\right)$ iteratively, where $\mathbf{x}$ is a pixel within the segmented foot and $k_{l}$ is the associated class label (with or without diabetic foot complications, in our case). The number of the desired classes (clusters) is predefined. Detailed about the EM clustering algorithm has been addressed in the previous Chapter. 
For this application, a simple model, a mixture of Gaussians with equal covariance matrices, was chosen and trained with the EM algorithm for each patient. This model is also referred to as EM with linear discriminated classifier (LDC). For pixels in the segmented foot area, the derived surface curvature (C) and the reconstructed surface albedo for the three colour channels $\left(\left[\rho_{r} \rho_{g}, \rho_{b}\right]\right)$ were combined and served as the input features for clustering. As such, for each pixel, $\mathbf{x}(u, v)=\left[C(u, v), \rho_{r}(u, v), \rho_{g}(u, v), \rho_{b}(u, v)\right]$, has a four dimensional feature space. Empirically, the desired number of classes was set to be 3, for discriminating whether a pixel with or without diabetic foot complications (2class problem). One extra class label was added mainly for the pixels that located at the board of the foot.

\subsection{Results and Discussion}

\subsubsection{PSI Reconstruction on Real Patient's Foot}

The PSI reconstruction was performed for the pixels on the plantar surface only utilising the above mentioned method. Reconstruction results, with the acquired images shown in Fig. 6.2, can be found in Fig. 6.4 and Fig. 6.5.

As stated before, it is hard to obtain the ground truth for the foot sole of a patient. But we can perceive the surface geometry changes presented in the surface normal map and the height map. Taking the patient shown in Fig.6.5 as example, the surface height map changes can be observed, at the positions of the callus (at the bottom of the hallux of the left foot in the image ), the ulceration regions on the right foot in the image), and the fold of skin on both feet.

Assume that segmented foot contours were always located on the same plane as the top surface of the sidelong supporting bars, the reconstructed heights for the pixels on the segmented foot contours were set to be zero. The foot sole surface heights were defined as the height differences between the reconstructed heights for pixels within the segmented foot region and the plane of the foot contours. Taking the heights of the highest pixel as the reconstructed foot elevation, the mean recovered foot sole surface elevation of all the 34 patients was $25 \pm 7 \mathrm{~mm}$.

For a quantitative evaluation, the reconstruction results of the spheres can be found in Fig. 6.6. By subtracting the height of the reconstructed background, the root mean square error (RMSE) of reconstructed height map of the entire surface of the half sphere (shown in Fig. 6.6(a)) is $0.5 \mathrm{~mm}$. However, if any 
movement was involved (about 30 pixels $(\sim 3 \mathrm{~mm})$ ) in acquired image set, without any registration step for correction of the movement the post PSI reconstruction, the reconstruction RMSE of the height can be as high as $4.6 \mathrm{~mm}$.

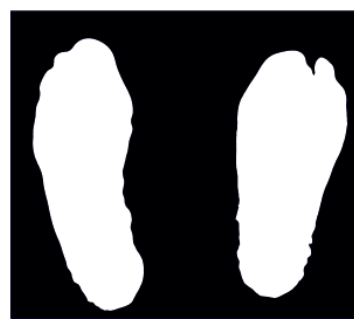

(a)

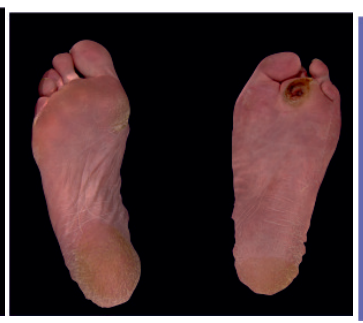

(b)

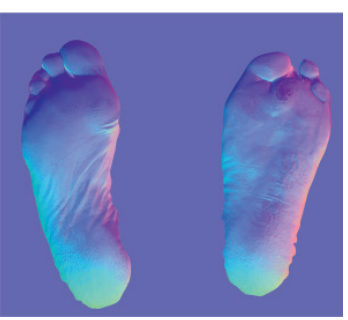

(c)

Figure 6.4: The Foot segmentation (a), the recovered surface albedo (b), and the RGB coded surface normal map (c). The RGB colour for the normal map was generated by normalising $\mathrm{x}, \mathrm{y}, \mathrm{z}$ comments in the interval of $[-1,1]$ to the $\mathrm{R}, \mathrm{G}, \mathrm{B}$ colour channel within interval $[0,1]$.

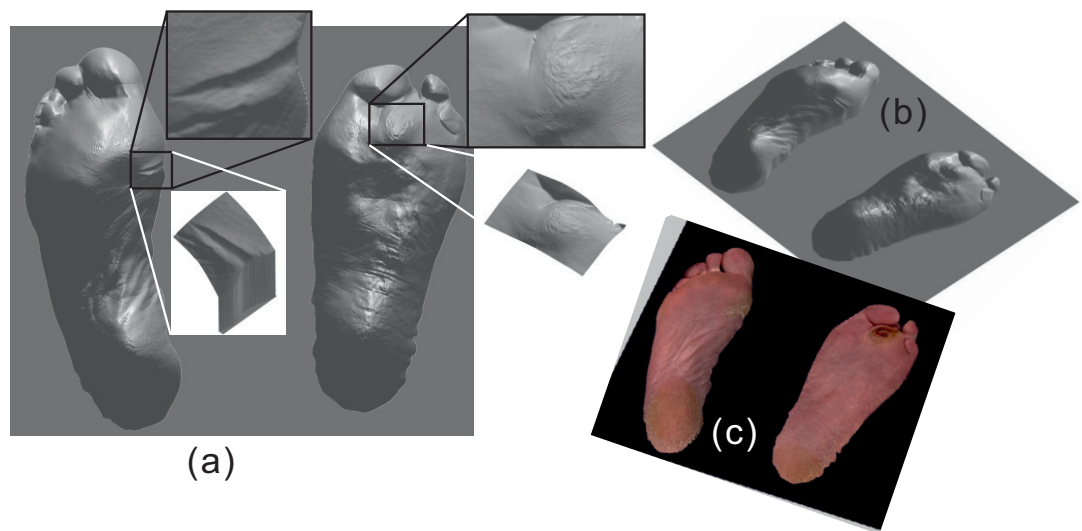

Figure 6.5: Reconstructed height map: (a) the top view of the foot sole, where the regions that had abundant callus and ulceration (indicated during live assessment by clinical expert) have been selected, zoomed and viewed in different angle. (b) the height map viewed from a different angle (c) the height map with textured colour. 


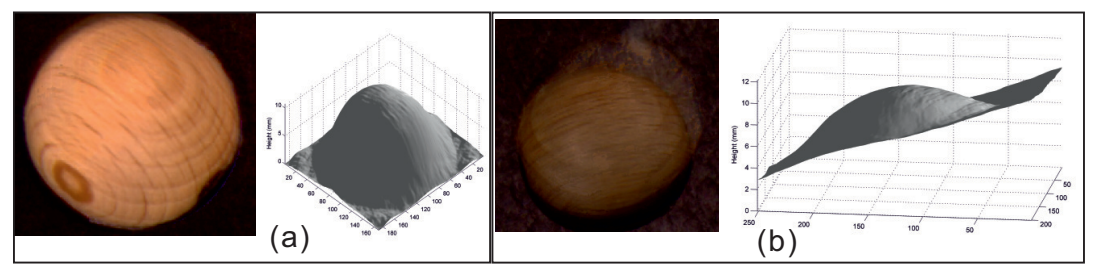

Figure 6.6: Reconstructed Surface Albedo and height map for the Spheres: (a) Sphere without movement involved in the image sequence acquisition. Relative height map of to its background, the highest point in the reconstructed height map $10.7 \mathrm{~mm}$. (b) Sphere with about 30 pixels $(\sim 3 \mathrm{~mm})$ movement involved. The movement can be observed in the recovered surface albedo.

\subsubsection{Detection of Diabetic Foot Complications}

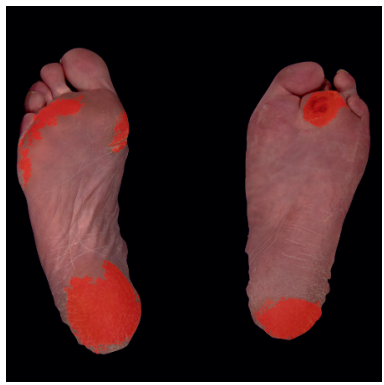

(a)

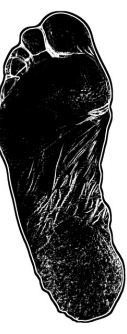

(b)
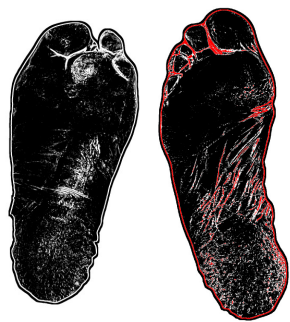

(c)

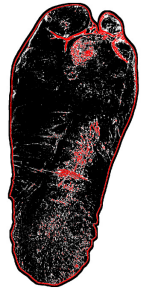

Figure 6.7: The region detected with EM clustering based on Surface Albedo and the depth curvature (a), the surface curvature map (b) and the region detected (Red) based on only the surface curvature (c).

With the recovered surface albedo and the surface curvature as as input features to EM algorithm for three clusters (this number was set empirically), the colour changes played a dormitory role in the clustering procedure. The colour changes on the heel and/or on the palms of the feet (shown in Fig. 6.7a) can also be detected, which accounts for small callus due to walking pressure. This is 'regular callus' on human foot but is yet a diabetic foot complication.

When using the surface curvature as input feature, surface geometry 
changes (edges) can be detected by thresholding the scalar curvature empirically. However, the detected edges (shown in Fig. 6.7c) do not only account for callus and ulceration, but also some from the skin fold. Taking only the curvature into consideration, simple thresholding was not able to distinguish the difference.

By comparing the segmentation results with the live assessment form from clinical experts, among the 34 patients in this preliminary study, there were 8 out of 9 ulcerations, 23 out of 27 callus, 4 out of 7 other foot complications (redness, necrosis and small defect) correctly detected. 12 regions with no signs of foot complications were labeled. As such, the sensitivity and specificity for the detection are $81.4 \%$ and $82.3 \%$, respectively.

\subsection{Conclusion}

In this contribution, a PSI system with 8 illumination sources was build and investigated for foot sole scanning and diabetic foot complications detection. When the imaging subject remains static during the image acquisition, the evaluated RMSE of height reconstruction from our image system was $0.5 \mathrm{~mm}$. This calibration was done with a half-sphere calibration target. To achieve such reconstruction accuracy, it requests that there is no foot movement during the image acquisition, which may be a burden for diabetic patients. So, in the future, foot registration should be implemented to eliminate the movement effects.

Currently, foot complications detection with EM clustering has $81.4 \%$ and $82.3 \%$ as sensitivity and specificity, respectively. However, it lacks the ability to distinguish the reasons for the colour or geometric changes, whether it comes from normal skin changes or accounts for diabetic foot complications. A supervised training technique, instead of unsupervised EM clustering, may be necessary to learn the classifiers the patterns of foot complications to differentiate the diabetic foot complications from the healthy skin more precisely, e.g. to differentiate normal callus from the abundant callus.

Moreover, the recovered surface albedo and the curvature were utilised as features for classification directly, both of which contained noise from the PSI reconstructions. Other image features, such as Gabor features and local binary patterns, and other 3D surface features, surface flatness for example, should be extracted and applied, which may make the detection less sensitivity to the noise.

Furthermore, more information may be provided by combining the 3D surface features, and the surface albedo, with the information achieved with other 
modalities in our experimental setup, e.g. thermal imaging [120], and multispectral imaging [40], to automatically detect diabetic foot complications and to differentiate among these complications. 


\section{Conclusion}

\subsection{Answers to Research Questions}

In this thesis, we have presented studies to detect diabetic foot complications with acquired images using an experimental setup. This experimental setup combined three imaging modalities, namely spectral imaging, infrared thermal imaging, and photometric stereo imaging.

Can skin spectra be used to detect diabetic foot complications, and to distinguish different foot complications?

The reflectance skin spectra may reveal the structure and properties of the skin tissue. In Chapter 3, we employed a spectroscopy system with a spectrometer to measure the region of interest that were assessed and annotated by clinical experts. Instead of deriving the underneath supporting physiological cause of the skin reflectance, machine learning technologies were applied to signify the differences between different foot complications. With a linear map of all the spectral information, the classification accuracy with the measured data to discriminate different diabetic foot complications may reach $90 \% \pm 10 \%$.

What are the most informative wavelength bands for the detection of diabetic foot complications and for distinguishing the different diabetic foot complications? Is it possible to build a Spectral Imaging system that employs these informative bands?

A data set with skin reflectance spectra from 227 skin regions from 64 patients was built and used for the optical filter selection. With the central wavelengths and pass-bands of the commercially available optical band-pass filters, the optical filters were simulated and applied to filter the skin spectra (measured with 
the spectrometer). The filters selection results show that the required optical filters for a spectral imaging system ranges from 3 to 7 , depending on additional constrains. With selected simulated filters sets, it was able to discriminate between ulcers, callus and healthy skin with a specificity of $96 \%$ and a sensitivity 97\%. Our stability analysis based on Monte Carlo method reveals that the shot noise of the designed imaging system would be a curtail factor, which may influence the classification performance.

Thus, it is feasible to build a spectral imaging system with a limited number of optical bandpass filters corresponding to the informative wavelength bands. This may firmly increase the measurement and cost efficiency of the future system.

\section{Can the spectral imaging system with the selected bandpass filters designed in our experimental setup detect different diabetic foot complications? What is the performance?}

In Chapter 4, an experimental setup with a 9-camera array fitted with 18 filters was built for spectral images acquisition from plantar foot of diabetic patients at high-risk for foot ulceration and amputation. Front-end pixel classifiers for automatic detection of the diabetic foot complications were trained and evaluated based on the annotation provided by clinical experts. Totally, 63 patients were measured and 62 'Callus', 29 'Ulceration', 11 'Rest', 6 'Fissure', 2 'Redness', 1 'Blister' regions and 369 'Healthy' regions were annotated. Among them, data from 42 patients were randomly selected as the training set from a dataset.

When a set of proper priors were chosen for quadratic discriminate classifiers training, the evaluation with the data from the other 21 patients provided classification performance of $78.8 \%$ sensitivity and $87.2 \%$ specificity for discriminate the skin pixels present or absent of diabetic foot complications. This result proved the concept that the designed front-end pixel classifier had the ability to detect the diabetic foot complications with acquired spectral images, which achieved the objective of the front-end pixel classifier designing.

However, $88 \%$ 'Callus' and only $65 \%$ of 'Ulceration' could be correctly detected with the trained pixel classifier. This indicated that the capability of the pixel classifier in discriminating the different foot complications was low; and the risk of miss detection was high. A back-end processing using the output of these classifiers is essential for the future study. In the back-end processing, higher level expert knowledge should be exploited to boost the performance to acceptable levels. 
Can infrared thermal imaging system in our experimental setup can be used for the detection of some of the diabetic foot complications?

In Chapter 5, a methodology was proposed for automated detection of diabetic foot complications with the acquired thermal images. The method was based on a simple asymmetric analysis combined with foot segmentation and landmark-based registration between the left and right foot. By assistance of the foot segmentation in digital colour images, the sensitivity and specificity of foot segmentation in the thermal image were $97.9 \% \pm 1.1 \%$ and $98.3 \% \pm 0.5 \%$, respectively, despite of the low contrast between the foot and the background in the thermal images. The left and right foot registration presented fairly good results regardless of the shapes, sizes, poses or positions of the two feet. Based on this left and right foot registration, the temperature difference of corresponding points on the contralateral feet could be easily achieved. As such, the diabetic foot complications could be identified by thresholding this temperature difference. The study in Chapter 5 provided a proof of concept that the thermal images acquired through our experimental setup can detect diabetic foot complications. However, this methodology is limited to patients, from whom both feet are available in the acquired images.

What kind of information can photometric stereo imaging provide in their application? Can photometric stereo imaging be used for the detection of diabetic foot complications?

In Chapter 6, a photometric stereo imaging system with 8 illumination sources in experimental setup was investigated for the foot sole scanning and diabetic foot complications detection. With the reconstructed foot depth map and surface albedo as input features, the 'Healthy' and suspicious 'unhealthy' regions on plantar foot was detected with Expectation-Maximisation (EM) clustering. The sensitivity and specificity of this unsupervised detection were $81.4 \%$ and $82.3 \%$, respectively. However, it lacks the ability to distinguish the reasons for the colour or geometric changes. In other words, EM clustering could detect the skin surface changes but it could not distinguish whether the changes come from normal skin (e.g. skin fold or foot pressure point) or account for diabetic foot complications. Therefore, a supervised training step may be necessary to learn the classifiers the patterns of foot complications. 


\subsection{Recommendations for Future Work}

\subsubsection{Spectral Imaging on Detection of Diabetic Foot Compli- cations}

Pioneer studies has implemented hyperspectral imaging system to monitor diabetic neuropathy [55], to assess the risk for ulceration formation [59] and to predict ulcer healing [56,57]. Most of the pioneer studies followed the 'analytical approach' [96], which seeks to deconstruct the reflectance spectra into biologically and physically meaningful factors, such as a map of tissue oxygenation. Despite of the valuable performance, the delicated hyperspectral imaging system is too expensive to be used at home environment.

Although study in Chapter 3 showed promising results in filter selection and detection different diabetic foot complications with skin reflectance data following a statistical approach, the 7 filters selected with the approach mounted in our experimental setup did not continue to provide good performance in signifying the difference between different diabetic foot complications. This may account for different illumination source (different spectral distribution) and different light distribution on the target surface. Besides, lacking of learning examples for the classifier training may be another explanation.

Further study with the spectra images should focus on the improvement of the detection, which might be achieved by:

- Including more patients for the training and testing, covering more variations within classes. This can also help us to eliminate the possible data correlation in classifier evaluation.

- Another way of eliminating the possible correlations would be to perform data grouping in a Gaussian pyramid.

- Perform a more delicate imaging system registration to taking both the intrinsic and extrinsic cameras properties into consideration. Reducing the misalignment could also improve the performance of the classifiers.

- The output of the trained pixel classifiers can be used as the input for a back-end processing in which hight level expert knowledge, such as the area and shapes of regions with foot complications, should be exploited to boost the performance to more satisfactory levels.

- Deriving physiological causal parameters from the measured spectra data based on a skin optical model. It may also improve the classification 
performance by adding the physiological parameters as input features.

\subsubsection{Thermal Imaging on Detection of Diabetic Foot Compli- cations}

The methodology for processing the acquired thermal images was intended to detect the diabetic foot complication by comparing the corresponding points on the contralateral feet. In finding the asymmetry of between left and right feet, registration was always done from left and right feet. This registration itself may introduce artificial asymmetry, which has not been investigated. In the future, both feet may be registered to a general and unbiased foot contour template to reduce such asymmetry.

The only criterion validated with randomised controlled trail (RCT) in temperature monitoring of diabetic foot is: if the temperature difference between the corresponding points on left and right foot is larger than $2.2^{\circ} \mathrm{C}$, the region is at risk for inflammation. However, recent studies by our fellow researchers pointed out that the $2.2^{\circ} \mathrm{C}$ criterion was not sufficient for different foot complications $[116,124]$. Besides, this comparison can not be conducted on patients with partial or entire foot amputated. Additionally, in case both feet have similar complications in corresponding regions, the complication will be missed as well.

Thus, the thermal image analysis should be further developed on ipsilateral foot to detect foot complications without the need to image the contralateral foot. One possibility for the ipsilateral foot temperature analysis is to compare the temperature with its neighbour pixels to find the hot spot at risk for inflammation.

\subsubsection{Photometric Stereo Imaging on Detection of Diabetic Foot Complications}

Photometric Stereo imaging was included into our experimental setup, intending to obtain a 3D reconstruction of the foot, provide more realistic presentation of the foot and detect the diabetic foot complications associated with texture changes.

However, this imaging modality is really sensitive to the object movement and illumination condition changes. For diabetic patients, it is hard to restrict the feet steady in the experimental setup during the image acquisition of the 8 images per patient, which has low light strength to avoid specular reflection 
and long exposure time for imaging. For the study in Chapter 6, around half of the patients were ruled out due to the foot movement and positioning.

A further study should be conducted to determine whether this modality is worthwhile for the following development of the telemedicine device.

- Improve the imaging system to unify illuminations and restrict the patient movement as much as possible.

- A robust algorithm for photometric stereo reconstruction should be developed, invariant of the object movement and illumination condition changes;

- Remove this modality from the further development. One alternative can be to use the 9 camera array geometry to reconstruct the 3D surface of plantar foot. In this way, the setup can get rid of one camera together with its eight illumination sources. With what, the system complexity will be reduced.

\subsubsection{Combination and Comparison Among the Three Imag- ing Modalities}

Currently, the information acquired with the three modalities in our experimental setup functioned in detecting diabetic foot complications separately. Although the results from the current studies indicated that each of them was promising for further development of the telemedicine system, a combination of the acquired information from the three may boost the performance and overcome their individual limitations.

Besides of the information integration, the adding values of each modality in detection of different diabetic complications should also be investigated and compared. If the function of one or two of the modalities in the performance improvement is so small that the modality/modalities can be reduced from the setup. In this way, the complexity of the future system will be reduced.

To do the combination and/or comparison between the three modalities, a dedicate imaging system registration is necessary. This registration can make sure the right pixel information be aligned with each other.

\subsubsection{Clinical Live Assessment and Medical Information}

Currently, only one clinical expert (either a wound consultant, surgeon or pediatrist) was involved in the live assessment of patient before image acquisition. 
Such assessment was done based on the personal education and experience. So, it would be quite subjective to the personal judgement with ineluctable bias. In the future, more clinicians should be involved for the live assessment to reduce such bias. In the meantime, an investment should be conducted to check the inter-clinician variation.

Furthermore, the live assessments were simply done on a paper form. Later in our data processing stage, our clinical researcher had to go back to the paper forms to annotated the foot for further usage, which was not efficient. In the future, a live annotation tool should be developed for clinicians to perform a digital live assessment.

Additionally, a complementary information database network might be helpful for the automatic diagnosis. Such a database may integrate all kinds of medical data together with the acquired images, such as the age, gender, types of diabetic, history of the ulceration, etc.

\subsubsection{Longitudinal Analysis}

The final objective of the project is to develop a telemedicine system which may predict the diabetic foot ulcerations. Therefore, a longitudinal study is desirable to monitor the development of the ulceration and the efficiency of the treatment. It is worth to mention that such study may require more time and arrangement of both the clinician and the patients. 



\section{Live Assessment Form}

Before the images acquisition started, any participated patient will be informed of the nature of the measurement. If they are consistent with the measurement protocol a patient consistence will be signed both by the patient and the clinical researcher, as shown in Fig. A.1.

Each foot was assessed by clinical expert for the presence of some foot complications, such as the presence of the ulceration, some import clinical pre-signs of ulceration (such as abundant callus, redness, fissures, blisters or other) or absence of any foot complications, on a simple live assessment form, as shown in Fig. A.2. 
Toestemmingsverklaring:

De ontwikkeling van een scanner voor de herkenning van problemen aan de diabetische voet

Ziekenhuisgroep Twente, locatie Almelo

Hierbij verklaar ik dat:

- Ik uitleg heb gekregen over het uit te voeren onderzoek.

Ik de schriftelijk informatie heb gelezen en begrepen.

- Ik aanvullende vragen heb kunnen stellen. Deze zijn genoeg beantwoord.

- $\quad$ Ik voldoende tijd heb gehad om te beslissen of ik meedoe.

- Ik begrijp dat al mijn persoonlijke gegevens strikt vertrouwelijk worden behandeld en anoniem worden verwerkt.

- Ik weet dat meedoen helemaal vrijwillig is en dat ik op ieder moment kan beslissen om toch niet mee te doen.

- $\quad$ Ik wil wel / niet nogmaals benaderd worden voor een volgende fase in dit onderzoek.

- Ik het bovenstaande heb gelezen, begrepen en stem toe met deelname aan het onderzoek 'De ontwikkeling van een scanner voor de herkenning van problemen aan de diabetische voet'.

Naam patiënt:

Geboortedatum: ....... - ...... - 19.....

Geslacht:

Huidige datum: ...20......

Handtekening:

Ik heb bovengenoemde deelnemer geïnformeerd over het onderzoek en hij/zij heeft zijn/haar bereidheid getoond om deel te nemen.

Naam onderzoeker:

Handtekening: Datum: ....... - ......- -..20.....

Figure A.1: Example of the patient consistence form. 
Appendix 1. Report form for live assessment to point out Regions of Interest (ROIs) 03

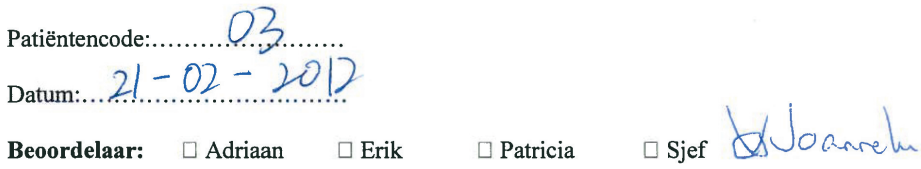

A.

Geef in onderstaande tekening duidelijk aan waar eventuele risicovolle plaatsen op de voetzool aanwezig zijn. Als criterium geldt dat de patiënt een oproep voor de polikliek zou krijgen. Geef de risicovolle locaties aan met de volgende codes:

$$
\begin{array}{ll}
1=\text { ulcus } & 4=\text { blaar } \\
2=\text { roodheid } & 5=\text { fissuur } \\
3=\text { overmatig callus } & 6=\text { anders }
\end{array}
$$

Of kruis aan 'geen bijzonderheden'

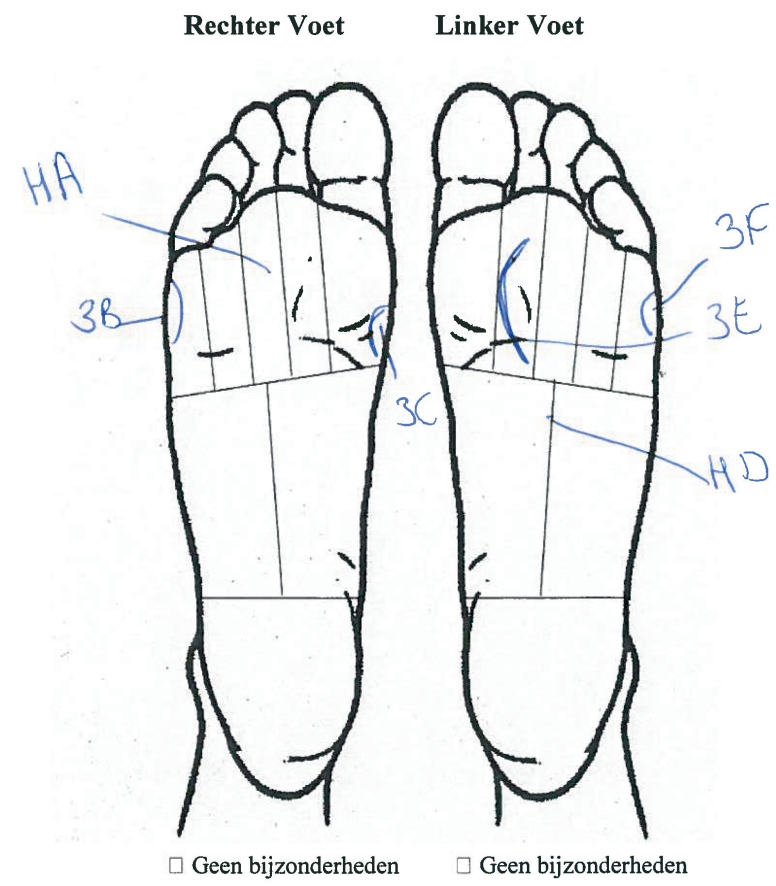

Figure A.2: Example of the live assessment form filled by clinical specialist. 



\section{Bibliography}

[1] S. Wild, G. Roglic, A. Green, R. Sicree, and H. King, "Global prevalence of diabetes: Estimates for the year 2000 and projections for 2030," Diabetes Care, vol. 27, no. 5, pp. 1047-1053, 2004.

[2] J. E. Shaw, R. A. Sicree, and P. Z. Zimmet, "Global estimates of the prevalence of diabetes for 2010 and 2030," Diabetes Research and Clinical Practice, vol. 87, no. 1, pp. 4-14, 2010.

[3] A. J. Boulton, L. Vileikyte, G. Ragnarson-Tennvall, and J. Apelqvist, “The global burden of diabetic foot disease," The Lancet, vol. 366, no. 9498, pp. 12-18, 2005.

[4] A. Boulton, D. Armstrong, S. F. Albert, R. Frykberg, R. Hellman, M. Kirkman, L. Lavery, J. W. LeMaster, J. L. Mills, M. J. Muller, P. Sheehan, and D. K. Wukich, "Comprehensive foot examination and risk assessment," Diabetes Care, vol. 31, no. 8, pp. 1679-1685, 2008.

[5] L. A. Lavery, K. R. Higgins, D. R. Lanctot, G. P. Constantinides, R. G. Zamorano, D. G. Armstrong, K. A. Athanasiou, and C. M. Agrawal, "Home monitoring of foot skin temperatures to prevent ulceration," Diabetes Care, vol. 27, no. 11, pp. 2642-2647, 2004.

[6] L. A. Lavery, K. R. Higgins, D. R. Lanctot, G. P. Constantinides, R. G. Zamorano, K. A. Athanasiou, D. G. Armstrong, and C. M. Agrawal, "Preventing diabetic foot ulcer recurrence in high-risk patients: use of temperature monitoring as a self-assessment tool," Diabetes Care, vol. 30, no. 1, pp. 14-20, 2007.

[7] J. Apelqvist, K. Bakker, W. van Houtum, and N. C. Schaper, "Practical guidelines on the management and prevention of the diabetic foot," Diabetes/Metabolism Research and Reviews, vol. 24, no. S1, pp. 225-231, 2008.

[8] I. D. Federation, "Idf diabetes altas sixth edition," on-Line, http://www.idf.org/ diabetesatlas.

[9] G. Chand, A. K. Mishra, S. Kumar, and A. Agarwal, "Diabetic foot," Clinical Queries: Nephrology, vol. 1, no. 2, pp. 144-150, 2012.

[10] N. Singh, D. G. Amstrong, and B. A. Lipsky, "Preventing foot ulcers in patients with diabetes," The Journal of the American Medical Association, vol. 293, no. 2, pp. 217-228, 2005. 
[11] G. E. Reiber, L. Vileikyte, and E. J. B. and, “Causal pathways for incident lowerextremity ulcers in patients with diabetes from two settings," Diabetes Care, vol. 22, no. 1, pp. 157-162, 1999.

[12] A. Boulton, "The diabetic foot: from art to science. the 18th camillo golgi lecture," Diabetologia, vol. 47, no. 8, pp. 1343-1353, 2004.

[13] V. Falanga, "Wound healing and its impairment in the diabetic foot," The Lancet, vol. 366, no. 9498, pp. 1736-1743, 2005.

[14] M. Lepäntalo, J. Apelqvist, C. Setacci, J. B. Ricco, G. de Donato, F. Becker, H. Robert-Ebadi, P. Cao, H. H. Eckstein, P. D. Rango, N. Diehm, J. Schmidli, M. Terra, F. L. Moll, F. Dick, and A. H. Davies, "Chapter v: Diabetic foot," European Journal of Vascular and Endovascular Surgery, vol. 42, no. S2, pp. S60-S74, 2011.

[15] J. J. Mendes and J. Neves, "Diabetic foot infections: Current diagnosis and treatment," The Journal of Diabetic Foot Complications, vol. 4, no. 2, pp. 26-45, 2012.

[16] A. Korzon-Burakowska and M. Edmonds, "Role of the microcirculation in diabetic foot ulceration," International Journal of Lower Extremity Wounds, vol. 5, no. 3, pp. 144-148, 2006.

[17] A. Boulton, "The diabetic foot: grand overview, epidermology and pathogenesis," Diabetes/Metabolism Research and Reviews, vol. 24, no. 1, pp. S3-S6, 2008.

[18] K. Bakker, J. Apelqvist, and N. C. Schaper, "Practical guidelines on the management and prevention of the diabetic foot 2011," Diabetes/Metabolism Research and Reviews, vol. 19, no. 1, pp. 377-384, 2012.

[19] S. Franc, A. Daoudi, S. Mounier, B. Boucherie, H. Laroye, C. Peschard, D. Dardari, O. Juy, E. Requeda, L. Canipel, and G. Charpentier, "Telemedicine: What more is needed for its integration in everyday life?" Diabetes $\mathcal{E}$ Metabolism, vol. 37, no. 4, pp. S71-S77, 2011.

[20] S. Franc, A. Daoudi, S. Mounier, B. Boucherie, D. Dardari, H. Laroye, B. Neraud, E. Requeda, L. Canipel, and G. Charpentier, "Telemedicine and diabetes: Achievements and prospects," Diabetes \& Metabolism, vol. 37, no. 6, pp. 463-476, 2011.

[21] R. Bellazzi, "Telemedicine and diabetes management: Current challenges and future research directions," Journal of Diabetes Science and Technology, vol. 2, no. 1, pp. 98-104, 2008.

[22] O. El-Gayar, P. Timsina, N. Nawar, and W. Eid, "A systematic review of it for diabetes self-management: Are we there yet?" International Journal of Medical Informatics, vol. 82, no. 8, pp. 637-652, 2013.

[23] L. Ratheau, N. Jeandidier, F. Moreau, S. Sigrist, and M. Pinget, "How technology has changed diabetes management and what is has failed to achieve," Diabetes $\mathcal{E}$ Metabolism, vol. 37, pp. S57-S64, 2011. 
[24] S. B. Larsen, J. Clemensen, and N. Ejskjaer, "A feasibility study of umts mobile phones for supporting nurses doing home visits to patients with diabetic foot ulcers," Journal of Telemedicine and Telecare, vol. 12, no. 7, pp. 358-362, 2006.

[25] G. E. Dafoulas, S. Koutsias, J. B. J. O. B. Malley, A. Gruentzig, L. A. Celi, P. Angelidis, K. Theodorou, and A. Giannoukas, "Development of an mhealth open source platform for diabetic foot ulcers tele-consultations," in Wireless Mobile Communication and Healthcare, ser. Lecture Notes of the Institute for Computer Sciences, Social Informatics and Telecommunications Engineering, K. S. Nikita, J. C. Lin, D. Fotiadis, and M.-T. A. Waldmeyer, Eds. Springer Berlin Heidelberg, 2012, vol. 83, pp. 158-164.

[26] J. Clemensen, S. B. Larsen, and N. Ejskjaer, "Telemedical treatment at home of diabetic foot ulcers," Journal of Telemedicine and Telecare, vol. 11, no. 2, pp. S2: 1416, 2005.

[27] J. Clemensen, S. B. Larsen, M. Kirkevold, and N. Ejskjaer, "Treatment of diabetic foot ulcerations in the home: Video consultations as an alternative to outpatient hospital care," International Journal of Telemedicine and Applications, vol. 2008, no. 132890, 2008.

[28] F. L. Bowling, L. King, H. Fadavi, J. A. Paterson, K. Preece, D. R. Matthews, and A. J. Boulton, "An assessment of the accuracy and usability of a novel optical wound measurement system," Diabetic Medicine, vol. 26, no. 1, pp. 93-96, 2009.

[29] F. L. Bowling, L. King, J. A. Paterson, J. Hu, B. A. Lipsky, D. R. Matthews, and A. J. Boulton, "Remote assessment of diabetic foot ulcers using a novel wound imaging system," Wound Repair and Regeneration, vol. 19, no. 1, pp. 25-30, 2011.

[30] S. A. Bus, C. E. V. B. Hazenberg, M. Klein, and J. G. Van Baal, “Assessment of foot disease in the home environment of diabetic patients using a new photographic foot imaging device," Journal of Medical Engineering \& Technology, vol. 34, no. 1, pp. 43-50, 2010.

[31] C. E. B. Hazenberg, J. G. van Baal, E. Manning, A. Bril, and S. A. Bus, “The validity and reliability of diagnosing foot ulcers and pre-ulcerative lesions in diabetes using advanced digital photography," Diabetes Technology \& Therapeutics, vol. 12, no. 12, pp. 1011-7, 2010.

[32] C. E. B. Hazenberg, S. A. Bus, A. I. Kottink, C. A. Bouwmans, A. M. SchöbachSpraul, and S. G. van Baal, "Telemedical home-monitoring of diabetic foot disease using photographic foot imaging - a feasibility study," Journal of Telemedicine and Telecare, vol. 18, no. 1, pp. 32-36, 2012.

[33] C. E. B. Hazenberg, J. J. van Netten, S. G. van Baal, and S. A. Bus, “Assessment of signs of foot infection in diabetes patients using photographic foot imaging and infrared thermography," Diabetes Technology \& Therapeutics, vol. 16, no. 6, p. [Epub ahead of print], 2014. 
[34] P. Ladyzynski, P. Foltynski, M. Molik, J. Tarwacka, K. Migalska-Musial, M. Mlynarczuk, J. M. Wojcicki, J. Krzymien, and W. Karnafel, "Area of the diabetic ulcers estimated applying a foot scanner-based home telecare system an three referenced methods," Diabetes Technology \& Therapeutics, vol. 13, no. 11, pp. 1011-1017, 2011.

[35] P. Foltynski, P. Ladyzynski, K. Migalska-Musial, S. Sabalinska, A. Ciechanowska, and J. Wojcicki, "A new imaging and data transmitting device for telemonitoring of diabetic foot syndrome patients," Diabetes Technology $\mathcal{E}$ Therapeutics, vol. 13, no. 8, pp. 861-867, 2011.

[36] P. Foltynski, J. M. Wojcicki, P. Ladyzynski, K. Migalska-Musial, G. Rosinski, J. Krzymien, and W. Karnafel, "Monitoring of diabetic foot syndrome treatment: some new aspectives," Artificial Organs, vol. 35, no. 2, pp. 176-182, 2011.

[37] A. Klein, "Alignment of diabetic feet," Master's thesis, University of Twente; Signals and Systems Group, Enschede, 2006, m.Sc. Report SAS2606.

[38] A. Klein, F. van der Heijden, and C. H. Slump, "Alignment of diabetic feet images," in Proceedings of SPS-DARTS 2007, the third annual IEEE Benelux/DSP Valley Signal Processing Symposium. Antwerp, Belgium: IEEE Benelux/DSP, 2007.

[39] S. Prahl, "Optical absorption of hemoglobin," last accessed September 11, 2014. [Online]. Available: http://omlc.ogi.edu/spectra/hemoglobin/index.html

[40] C. Liu, J. J. van Netten, M. E. Klein, J. G. van Baal, S. A. Bus, and F. van der Heijden, "Statistical analysis of spectral data: a methodology for designing an intelligent monitoring system for the diabetic foot," Journal of Biomedical Optics, vol. 18, no. 12, p. 126004, 2013.

[41] K. J. Zuzak, M. D. Schaeberle, E. N. Lewis, and I. W. Levin, “Visible reflectance hyperspectral imaging: Characterization of a noninvasive, in vivo system for determining tissue perfusion," Analytical Chemistry, vol. 74, no. 9, pp. 2021-2028, 2002.

[42] T. Vo-Dinh, "Development of a multi-spectral imaging system for medical applicatons," Journal of Physics D: Applied PhysicsEmail alert RSS feed, vol. 36, no. 14, pp. 1663-1668, 2003.

[43] T. Vo-Dinh, D. L. Stokes, M. B. Wabuyele, M. E. Martin, J. M. Song, R. Jagannathan, E. Michaud, R. J. Lee, and X. pan, "A hyperspectral imaging system for in vivo optical diagnosis," IEEE Engineering in Medicine and Biology Magazine, vol. 23, pp. 40-49, 2004.

[44] T. Dinh, F. Tecilazich, A. Kafanas, J. Doupis, C. Gnardellis, E. Leal, A. Tellechea, L. Pradhan, T. E. Lyons, J. M. Giurini, and A. Veves, "Mechanisms involved in the development and healing of diabetic foot ulceration," Diabetes, vol. 61, no. 11, pp. 2937-2947, 2012. 
[45] M. J. C. van Gemert, S. L. Jacques, H. J. C. M. Sterenborg, and W. M. Star, "Skin optics," IEEE transactions on Biomedical Engineering, vol. 36, no. 12, pp. 1146-1154, 1989.

[46] V. V. Tuchin, Tissue Optics: Light Scattering Methods and Instruments for Medical Diagnosis, 2nd ed. SPIE Publications, 2007.

[47] T. Lister, P. A. Wright, and P. H. Chappell, "Optical properties of human skin," Journal of Biomedical Optics, vol. 17, no. 9, p. 090901, 2012.

[48] A. Korzon-Burakowska and M. Edmonds, "Role of the microcirculation in diabetic foot ulceration," International Journal of Lower Extremity Wounds, vol. 5, pp. 144-148, 2006.

[49] G. Rayman, S. A. Williams, P. D. Spencer, L. H. Smaje, P. H. Wise, and J. E. Tooke, "Impaired microvascular hyperaemic response to minor skin trauma in type i diabetes." British medical journal (Clinical research ed.), vol. 292, no. 6531, pp. 12951298, 1986.

[50] E. S. Papazoglou, M. S. Weingarten, L. Zubkov, L. Zhu, S. Tyagi, and K. Pourrezaei, "Optical properties of wounds: Diabetic versus optical properties of wounds: Diabetic versus healthy tissue," IEEE transactions on Biomedical Engineering, vol. 53, no. 6, pp. 1047-1055, 2006.

[51] E. S. Papazoglou, M. S. Weingarten, L. Zubkov, M. Neidrauer, L. Zhu, S. Tyagi, and K. Pourrezaei, "Changes in optical properties of tissue during acute wound healing in an animal model," Journal of Biomedical Optics, vol. 13, no. 4, p. 044005, 2008.

[52] E. S. Papazoglou, "Noninvasive assessment of diabetic foot ulcers with diffuse photon density wave methodology: pilot human study," Journal of Biomedical Optics, vol. 14, no. 6, p. 064032, 2009.

[53] M. Neidrauer, L. Zubkov, M. S. Weingarten, K. Pourrezaei, and E. S. Papazoglou, "Near infrared wound monitor helps clinical assessment of diabetic foot ulcers," Journal of Diabetes Science and Technology, vol. 4, no. 4, pp. 792-798, 2010.

[54] M. S. Weingarten, J. A. Samuels, M. Neidrauer, X. Mao, D. Diaz, J. McGuire, J. McDaniel, L. Jenkins, L. Zubkov, and E. S. Papazoglou, "Diffuse near-infrared spectroscopy prediction of healing in diabetic foot ulcers: A human study and cost analysis," Wound Repair and Regeneration, vol. 20, no. 6, pp. 911-917, 2012.

[55] R. L. Greenman, S. Panasyuk, X. Wang, T. E. Lyons, T. Dinh, L. Longoria, J. M. Giurini, J. Freeman, L. Khaodhiar, and A. Veves, "Early changes in the skin microcirculation and muscle metabolism of the diabetic foot," The Lancet, vol. 366, no. 9498, pp. 1711-1717, 2005.

[56] L. Khaodhiar, T. Dinh, K. T. Schomacker, S. V. Panasyuk, J. E. Freeman, R. Lew, T. Vo, A. A. Panasyuk, C. Lima, J. M. Giurini, T. E. Lyons, and A. Veves, "The 
use of medical hyperspectral technology to evaluate microcirculatory changes in diabetic foot ulcers and to predict clinical outcomes," Diabetes Care, vol. 30, no. 4, pp. 903-910, 2007.

[57] A. Nouvong, B. Hoogwerf, E. Mohler, B. Davis, A. Tajaddini, and E. Medenilla, "Evaluation of diabetic foot ulcer healing with hyperspectral imaging of oxyhemoglobin and deoxyhemoglobin," Diabetes Care, vol. 32, no. 11, pp. 2056-2061, 2009.

[58] D. Yudovsky, A. Nouvong, K. Schomacker, and L. Pilon, “Monitoring temporal development and healing of diabetic foot ulceration using hyperspectral imaging," Journal of Biophotonics, vol. 4, no. 7-8, pp. 565-576, 2011.

[59] — " "Assessing diabetic foot ulcer development risk with hyperspectral tissue oximetry," Journal of Biomedical Optics, vol. 16, no. 2, pp. (026 009) 1-8, 2011.

[60] S. J. Benbow, A. W. Chan, D. R. Bowsher, G. William, and I. A. Macfarlane, “The prediction of diabetic neuropathic plantar foot ulceration by liquid-crystal contact thermography," Diabetes Care, vol. 17, no. 8, pp. 835-839, 1994.

[61] D. G. Armstrong and L. A. Lavery, "Monitoring neuropathic ulcer healing with infrared dermal thermometry," Journal of Foot and Ankle Surgery, vol. 35, no. 4, pp. 335-338, 1996.

[62] D. G. Armstrong, L. A. Lavery, P. J. Liswood, W. F. Tood, and J. A. Tredwell, "Infrared dermal thermometry for the high-risk diabetic foot," Physical Therapy, vol. 77, no. 2, pp. 169-175, 1997.

[63] D. G. Armstrong, K. Holtz-Neiderer, C. Wendel, M. J. Mohler, H. R. Kimbriel, and L. A. Lavery, "Skin temperature monitoring reduces the risk for diabetic foot ulceration in high-risk patients," The American Journal of Medicine, vol. 120, no. 12, pp. 1042-1046, 2007.

[64] M. Bharara, J. E. Cobb, and D. J. Claremont, "Thermography and thermometry in the assessment of diabetic neuropathic foot: A case for furthering the role of thermal techniques," International Journal of Lower Extremity Wounds, vol. 5, no. 4, pp. 250-260, 2006.

[65] M. Bharara, J. Schoess, A. Nouvong, and D. G. Armstrong, "Wound inflammatory index: a "proof of concept" study to assess wound healing trajectory," Journal of Diabetes Science and Technology, vol. 4, no. 4, pp. 773-779, 2010.

[66] K. Roback, M. Johansson, and A. Starkhammar, "Feasibility of a thermographic method for early detection of foot disorders in diabetes," Diabetes Technology $\mathcal{E}$ Therapeutics, vol. 11, no. 10, pp. 663-337, 2009.

[67] P. Sun, H. Lin, S. H. Jao, Y. C. Ku, R. C. Chan, and C. K. Cheng, "Relationship of skin temperature to sympathetic dysfunction in diabetic at-risk feet," Diabetes Research and Clinical Practice, vol. 73, no. 1, pp. 41-46, 2006. 
[68] P. C. Sun, H. D. Lin, S. H. Jao, R. C. Chan, M. J. Kao, and C. K. Cheng, "Thermoregulatory sudomotor dysfunction and diabetic neuropathy develop in parallel in at-risk feet," Diabetic Medicine, vol. 25, no. 3, pp. 413-418, 2008.

[69] R. G. Frykberg, A. Tallis, and E. Tierney, "Diabetic foot self examination with the tempstat ${ }^{\mathrm{U}}$ as an integral component of a comprehensive prevention program," The Journal of Diabetic Foot Complications, vol. 1, no. 1, pp. 13-18, 2009.

[70] K. Nishide, T. Nagase, M. Oba, M. Oe, Y. Ohashi, S. Iizaka, G. Nakagami, T. Kadowaki, and H. Sanada, "Ultrasonographic and thermographic screening for latent inflammation in diabetic foot callus," Diabetes Research and Clinical Practice, vol. 85, no. 3, pp. 304-309, 2009.

[71] T. Nagase, H. Sanada, K. Takehara, M. Oe, S. Iizaka, Y. Ohashi, M. Oba, T. Kadowaki, and G. Nakagami, "Variations of plantar thermographic patterns in normal controls and non-ulcer diabetic patients: novel classification using angiosome concept." Journal of Plastic, Reconstructive E Aethetic Surgery, vol. 64, no. 7, pp. 860-866, 2011.

[72] M. Bharara, J. Schoess, and D. G. Armstrong, "Coming events cast their shadows before: detecting inflammation in the acute diabetic foot and the foot in remission," Diabetes/Metabolism Research and Reviews, vol. 28, no. 1, pp. 15-20, 2012.

[73] N. Kaabouch, Y. Chen, W.-C. Hu, J. W. Anderson, F. Ames, and R. Paulson, “Enhancement of the asymmetry-based overlapping analysis through features extraction," Journal of Electronic Imaging, vol. 20, no. 1, pp. 013 012-013 012-7, 2011.

[74] N. Kaabouch, W.-C. Hu, and Y. Chen, "Alternative technique to asymmetry analysis-based overlapping for foot ulceration examination: Scalable scanning," Journal of Diabetes and Metabolism, vol. S5, p. 003, 2011.

[75] S. Seitz, B. Curless, J. Diebel, D. Scharstein, and R. Szeliski, "A comparison and evaluation of multi-view stereo reconstruction algorithms," in Computer Vision and Pattern Recognition, 2006 IEEE Computer Society Conference on, vol. 1, June 2006, pp. 519-528.

[76] D. Nehab, S. Rusinkiewicz, J. Davis, and R. Ramamoorthi, "Efficiently combining positions and normals for precise $3 \mathrm{~d}$ geometry," ACM Transactions on Graphics, vol. 24, no. 3, pp. 536-543, Juli 2005.

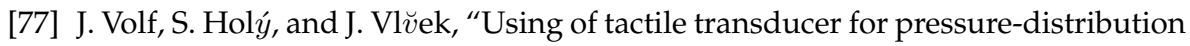
measurement on the sole of the foot," Sensors and Actuators A: Physical, vol. 62, no. 1-3, pp. 556 - 561, 1997, proceedings of Eurosensors X.

[78] R. J. Woodham, "Photometric method for determing surface orientation from multiple images," Optical Engineering, vol. 19, no. 1, pp. 139-144, 1980.

[79] S. Barsky and M. Petrou, "The 4-source photometric stereo technique for threedimensional surfaces in the presence of highlights and shadows," IEEE Transactions on Pattern Analysis and Machine Intelligence, vol. 25, no. 10, pp. 1239-1252, 2003. 
[80] __ "Design issues for a colour photometric stereo system," Journal of Mathematical Imaging and Vision, vol. 24, no. 1, pp. 143-162, 2006.

[81] J. Sun, M. Smith, L. Smith, S. Midha, and J. Bamber, “Object surface recovery using a multi-light photometric stereo technique for non-lambertian surfaces subject to shadows and specularities," Image and Vision Computing, vol. 25, no. 7, pp. 1050-1057, 2007.

[82] V. Argyriou, M. Petrou, and S. Barsky, "Photometric stereo with an arbitrary number of illuminants," Computer Vision and Image Understanding, vol. 114, no. 8, pp. 887-900, 2010. [Online]. Available: \url\{http://www.sciencedirect.com/ science/article/B6WCX-5051PS9-1/2/7d6b74dc88c1c4f53ce50e41d7b33657\}

[83] C. Liu, F. van der Heijden, and J. J. van Netten, "Towards surface analysis on diabetic feet soles to predict ulcerations using photometric stereo." in Proceedings of SPIE 8214, Advanced Biomedical and Clinical Diagnostic Systems X, vol. 82141D, 2012.

[84] J. Apelqvist, K. Bakker, W. H. van Houtum, M. H. Nabuurs-Franssen, and N. C. Schaper, "International consensus and practical guidelines on the management and the prevention of the diabetic foot," Diabetes/Metabolism Research and Reviews, vol. 16, no. 1, pp. S84-S92, 2000.

[85] W. Li, "Locality-preserving dimensionality reduction and classification for hyperspectral image analysis," IEEE Transactions on Geoscience and Remote Sensing, vol. 50, no. 4, pp. 1185-1198, 2012.

[86] F. van der Heijden, R. P. W. Duin, D. de Ridder, and D. M. J. Tax, Classification, Parameter Estimation and State Estimation An Engineering Approach Using MATLAB. John Wiley \& Sons, 2004.

[87] "Edmund optics catalogue of the interfernce bandpass filters from 400nm to 1000nm," last accessed September 11, 2014. [Online]. Available: http: //www.edmundoptics.com/optics/optical-filters/

[88] "Comar optics catalogue of the interfernce bandpass filters from 400nm to 1000nm," last accessed September 11, 2014. [Online]. Available: http: //www.comaroptics.com/pdfs/

[89] T. M. Cover, "Geometrical and statistical properties of systems of linear inequalities with applications in pattern recognition," IEEE Transactions on Electronic Computers, vol. EC-14, pp. 326-334, 1965.

[90] R. Kohavi and G. H. John, "Wrappers for feature subset selection," Artificial Intelligence, vol. 97, pp. 273-324, 1997.

[91] A. K. Jain, R. P. W. Duin, and J. Mao, "Statistical pattern recognition: A review," IEEE Transactions on Pattern Analysis and Machine Intelligence, vol. 22, pp. 4-37, 2000. 
[92] D. Yudovsky and L. Pilon, "Rapid and accurate estimation of blood saturation, melanin content, and epidermis thickness from spectral diffuse reflectance," $A p$ plied Optics, vol. 49, no. 10, pp. 1707-1719, 2010.

[93] T. Vo-Dinh, W. S. Grundfest, and A. Mahadevan-Jansen, Eds., Two-layer Optical Model of Skin for Early, Non-invasive Detection of Wound Development on the Diabetic Foot, ser. Advanced Biomedical and Clinical Diagnostic Systems VIII, vol. 7555, no. (755514-)1-10, SPIE. SPIE, 2010.

[94] P. Regtien, F. van der Heijden, M. J. Korsten, and W. Otthius, Measurement Science for Engineers. Butterworth-Heinemann, 2004, ch. 10, pp. 277-307.

[95] M. Bharara, J. L. Mills, K. Suresh, H. L. Rilo, and D. G. Armstrong, “Diabetes and landmine-related amputations: a call to arms to save limbs," International Wound Journal, vol. 6, no. 1, pp. 2-3, 2009.

[96] V. V. Tuchin, Ed., Handbook of Optical Biomedical Diagnostics. SPIE Press, 2002.

[97] D. G. Lowe, "Object recognition from local scale-invariant features," in The Proceedings of the Seventh IEEE International Conference on Computer Vison, 1999, pp. 1150-1157.

[98] _ "Local feature view clustering for 3d object recognition," in Proceedings of the 2001 IEEE Computer Society Conference on Computer Vision and Pattern Recognition (CVPR 2001), vol. 1, 2001, pp. 682-688.

[99] —_ "Distinctive image features from scale-invariant keypoints," International Journal of Computer Vision, vol. 60, no. 2, pp. 91-110, 2004.

[100] K. Mikolajzyk and C. Schmid, "A performance evaluation of local descriptors," IEEE Transactions on Pattern Analysis and Machine Intelligence, vol. 27, no. 10, pp. 1615-1630, 2005.

[101] M. A. Fischler and R. C. Bolles, "Random sample consensus: a paradigm for model fitting with applications to image analysis and automated cartography," Communications of the ACM, vol. 24, no. 6, pp. 381-395, 1981.

[102] D. G. Lowe, "Demo software: Sift keypoint detector," 2005, last accessed September 11, 2014. [Online]. Available: http:/ / www.cs.ubc.ca/ lowe/keypoints /

[103] Z. Yi, C. Zhiguo, and X. Yang, "Multi-spectral remote image registration based on sift," Electronics Letters, vol. 44, no. 2, pp. 107-108, 2008.

[104] H. Gonçalves, L. Corte-Real, and J. A. Gonçalves, "Automatic image registration through image segmentation and sift," IEEE Transactions on Geoscience and Remote Sensing, vol. 49, no. 7, pp. 2589-2600, 2011.

[105] B. Jähne, Practical Handbook on Image Processing for Scientific Applications. CRC Press, 1997.

[106] N. Otsu, "A threshold selection method from gray-level histograms," IEEE Transcations on Systems, Man and Cybernetics, vol. 9, no. 1, pp. 62-66, 1979. 
[107] X.-H. Zhou, N. A. Obuchowski, and D. K. McClish, Statistical Methods in Diagnostic Medicine, 2nd ed. Wiley, 2011, ch. 2 Measures of Diagnostic Accuracy, pp. $13-54$.

[108] T. Fawcett, "An introduction to roc curve," Pattern Recognition Letters, vol. 26, no. 8, pp. 861-874, 2006.

[109] A. P. Bradley, "The use of the area under the roc curve in the evaluation of machine learning algorithm," Pattern Recognition, vol. 30, no. 7, pp. 1145-1159, 1997.

[110] R. Kumar and A. Indrayan, "Receiver operating characteristic (roc) curve for medical researchers," Indian Pediatrics, vol. 48, no. 4, pp. 277-288, 2011.

[111] P. Agache and P. Humbert, Eds., Measuring the Skin. Springer, 2004.

[112] C. Liu, F. van der Heijden, M. E. Klein, J. G. van Baal, S. A. Bus, and J. J. van Netten, "Infrared dermal thermography on diabetic feet soles to predict ulcerations: a case study," in Advanced Biomedical and Clinical Diagnostic Systems XI, Proceedings of SPIE 8572, 2013, p. 85720N.

[113] D. Rueckert, L. I. Sonoda, C. Hayes, D. L. G. Hill, M. O. Leach, and D. J. Hawkes, "Nonrigid registration using free-form deformations: Application to breast mr images," IEEE Transcations on Medical Imaging, vol. 18, no. 8, pp. 712-721, 1999.

[114] D. J. Kroon, "Segmentation of the mandibular canal in cone-beam ct data," $\mathrm{PhD}$ Dissertation, MIRA Institute for Biomedical Technology and Technical Medicine, University of Twente, Enschede, The Netherlands, 2011.

[115] _ , "B-spline grid, image and point based registration," last accessed September 11, 2014. [Online]. Available: http://www.mathworks.com/matlabcentral/ fileexchange/20057-b-spline-grid-image-and-point-based-registration

[116] J. J. van Netten, J. G. van Baal, C. Liu, F. van der Heijden, and S. A. Bus, “Infrared thermal imaging for automated detection of diabetic foot complications," Journal of Diabetes Science and Technology, vol. 7, no. 5, pp. 1122-1129, 2013.

[117] P. Kakumanu, S. makrogiannis, and N. Bourbakis, "A survey of skin-color modeling and detection methods," Pattern Recognition, vol. 40, no. 3, pp. 1106-1122, 2007.

[118] N. Vandenbroucke, L. Macaire, and J.-G. Postaire, “Color image segmentation by pixel classification in an adpated hybrid color space. application to soccer image analyis," Computer Vision and Image Understanding, vol. 90, no. 2, pp. 190-216, 2003.

[119] J.-Y. Bouguet, "Camera calibration toolbox for matlab." [Online]. Available: http://www.vision.caltech.edu/bouguetj/calib\_doc/

[120] C. Liu, J. J. van Netten, J. G. van Baal, S. A. Bus, and F. van der Heijden, “Automatic detection of diabetic foot complications with infrared thermography by asymmetric analysis," 2014, submitted to the IEEE Transactions on Medical Imaging. 
[121] H. Rushmeier, G. Taubin, and A. Guéziec, “Applying shape from lighting variation to bump map capture," in Eighth Eurographics Rendering Workshop, 1997, pp. 53-44.

[122] R. T. Frankot and R. Chellappa, "A method for enforcing integrability in shape from shading algorithm," IEEE Transactions on Pattern Analysis and Machine Intelligence, vol. 10, no. 4, pp. 439-451, July 1988.

[123] R. J. Woodham, "Gradient and curvature from the photometric-stereo method, including local confidence estimation," Journal of optical Society of America, vol. A 11, pp. 3050-3068, 1994.

[124] J. J. van Netten, M. Prijs, Jeff, G. van Baal, C. Liu, F. van der Heijden, and S. A. Bus, "Diagnostic values of temperature assessment for detection of diabetic foot complications," 2014, accepted by the Journal of Diabetes Research. 



\section{List of Publications}

- Chanjuan Liu, Jaap J. van Netten, Jeff G. van Baal, Sicco A. Bus, and Ferdi van der Heijden, "A Front-end for Automatic Detection of Diabetic Foot Complications using Spectral Images: a Statistical Approach to Pixel Based Segmentation", ready for submission

- Chanjuan Liu, Jaap J. van Netten, Jeff G. van Baal, Sicco A. Bus, and Ferdi van der Heijden, "Photometric Stereo Imaging to Reconstruct the 3D Surface of the Plantar Foot of Diabetic Patients: A Feasibility Study on Detection of Diabetic Foot Complications", ready for submission

- Chanjuan Liu, Jaap J. van Netten, Jeff G. van Baal, Sicco A. Bus, and Ferdi van der Heijden, "Automatic detection of diabetic foot complications with infrared thermography by asymmetric analysis," 2014, submitted.

- Chanjuan Liu, Jaap J. van Netten, Marvin E. Klein, Jeff G. van Baal, Sicco A. Bus, and Ferdi van der Heijden, "Statistical analysis of spectral data: a methodology for designing an intelligent monitoring system for the diabetic foot," Journal of biomedical optics, vol. 18, no. 12, p. 126004, 2013.

- Chanjuan Liu, Ferdi van der Heijden, Marvin E. Klein, Jeff G. van Baal, Sicco A. Bus, and Jaap J. van Netten, "Infrared dermal thermography on diabetic feet soles to predict ulcerations: a case study," in Advanced Biomedical and Clinical Diagnostic Systems XI, Proceedings of SPIE 8572, 2013, p. 85720N.

- Chanjuan Liu, Ferdi van der Heijden, and Jaap J. van Netten, “Towards surface analysis on diabetic feet soles to predict ulcerations using photometric stereo." in Proceedings of SPIE 8214, Advanced Biomedical and Clinical Diagnostic Systems X, vol. 82141D, 2012.

- Jaap J. van Netten, Jeff G. van Baal, Chanjuan Liu, Ferdi van der Heijden, and Sicco A. Bus, "Infrared thermal imaging for automated detection of diabetic foot complications," Journal of diabetes science and technology, vol. 7, no. 5, pp. 1122-1129, 2013.

- Jaap J. van Netten, Miranda Prijs, Jeff G. van Baal, Chanjuan Liu, Ferdi van der Heijden, and Sicco A. Bus, "Diagnostic values of temperature assessment for detection of diabetes-related foot complications", accepted by Diabetes Technology and Therapeutics, 2014 



\section{Summary}

Diabetes Mellitus (DM) is one of the most common chronic diseases globally. Currently more than 382 million people are diagnosed with DM. The population and significance of DM are expected to rise even further in the future. People with DM are at risk of developing a number of disabling and life-threatening health problems, such as diabetic foot complications, including ulceration and its pre-signs (e.g. abundant callus, inflammation, fissures, blisters). Approximately $25 \%$ of patients with DM eventually develop foot ulcers. If not treated in time, the risk for (partial) lower extremity amputations or mortality increases. These devastating consequences can be prevented by early detection and timely treatment of the diabetic foot complications. This early identification strongly depends on frequent risk assessment, preferably on a daily basis, especially for high-risk patients. However, frequent assessment by healthcare professionals is costly and not always possible. Self-examination by patients is difficult and impractical, due to other complications (e.g. limited joint mobility and reduced eyesight).

An intelligent telemedicine system, which is compact, non-invasive, non-contact and user-friendly, may provide a solution for frequent assessment, which is the ultimate objective of our project. As the first step to approach this goal, an experimental setup that combined three promising imaging modalities, namely spectral imaging, infrared thermal imaging, and photometric stereo imaging, was developed and investigated.

A spectral imaging system consisting of nine cameras in a matrix configuration was developed, fitted with the preselected optical filters. This pre-selection of the filters was conducted to obtain the most informative bandpass filters that signified the difference between different skin conditions. Using the spectral images acquired, front-end pixel classifiers were developed to detect diabetic foot complications, taking image annotations based on the live assessment as the ground truth. These front-end pixel classifiers can distinguish presence or absence of diabetic foot complications with acceptable performance. However, they are lack of the capability of differentiating between the various diabetic foot complications. Future studies are needed on enhancing the performance of current pixel classifiers and designing the back-end classifiers.

With the infrared thermal imaging, the temperature difference between corresponding points on the left and right foot in the thermal images were compared to detect the risk of inflammation. As the temperature contrast between the feet and background was low, it was hard to perform the foot segmentation directly from the thermal images. To issue this, foot segmentations from digital color images were conducted for assistance. The segmented left and right foot were then registered with each other, which which helped to compare the temperature of the corresponding points on the contralateral feet. The outcomes of the thermal image analysis proved to be promising in the early detection of foot complications in patients with DM and patients who are at high risk 
for these complications. However, this methodology is limited to patients, from whom both feet are available in the acquired images.

With photometric stereo imaging, a feasibility study was conducted to detect diabetic foot complications. The surface curvature (derived from the reconstructed surface normal) and the reconstructed surface albedo were treated as input features to distinguish the skin regions with or without diabetic foot complications. The results indicate that this imaging technology is promising, although it still has some limitations currently, such as the movement in patients' foot during image acquisition. To determine the potential value of this modality in the future telemedicine system, further improvement is required.

The outcomes of the studies presented in this thesis showed the feasibility of developing a telemedicine system to detect diabetic foot complications with the three imaging modalities. The studies acted as the precursors for developing such an intelligent telemedicine system, which proposed potential detection methodologies and provided the directions for the future study. Following the conclusion and recommendations of this thesis, we expect an intelligent telemedicine system suitable for measurements at the patients' homes or at health centres can be developed in the future. 


\section{Samenvatting}

Diabetes Mellitus (DM) is wereldwijd een van de meest voorkomende chronische ziekten. Op dit moment zijn meer dan 382 miljoen mensen gediagnosticeerd met DM. Er wordt verwacht dat de populatie en significantie van DM in de toekomst alleen maar verder stijgen. Mensen met DM lopen het risico op verschillende beperkende en levensbedreigende gezondheidsproblemen. Een voorbeeld hiervan zijn diabetische voet complicaties, zoals ulcera en de voortekenen van ulcera (bijv. overmatig callus, inflammatie, fissuren, blaren). Ongeveer $25 \%$ van de patiënten met DM ontwikkelen gedurende hun leven een voetulcus. Als deze niet tijdig behandeld worden stijgt het risico op amputatie van (delen van) de onderste extremiteit en mortaliteit. Deze verwoestende consequenties kunnen voorkomen worden door vroege herkenning en tijdige behandeling van diabetische voet complicaties. Deze vroege herkenning is in sterke mate afhankelijk van frequente risicobeoordeling, bij voorkeur dagelijks, vooral bij patiënten met een hoog risico. Echter, frequente beoordeling door gezondheidsprofessionals is kostbaar en niet altijd mogelijk. Beoordeling door patiënten zelf is moeilijk en niet praktisch, mede door de andere complicaties die deze patiënten hebben (bijv. beperkte mobiliteit in de gewrichten en verminderd zicht).

Een intelligent telemedicine systeem, dat compact is, niet invasief, geen contact maakt met de voet en gebruiksvriendelijk is, kan een oplossing zijn voor frequente beoordeling. Een dergelijk systeem is het uiteindelijke doel van dit project. Als eerste stap om dit doel te bereiken is een experimentele setup ontwikkeld en onderzocht. Deze setup bestaat uit een combinatie van drie veel belovende modaliteiten, namelijk "spectrale imaging", "thermische imaging", en "photometrische stereo imaging".

Het "spectral imaging" systeem, bestaande uit negen camera's in een matrix configuratie was ontwikkeld, waarbij met behulp van optische filters de gewenste spectra werd geselecteerd. Deze selectie werd gebruikt om tot de juiste selectie van optische filters te bepalen, welke gebruikt worden voor de verschillende huid-aandoeningen. Gebruik makend van de spectrale beelden, "front-end pixel classifiers" werden ontwikkeld om de complicaties van diabetische voeten te detecteren, door annotaties te gebruiken gebaseerd op de live assessment die de "ground-truth" bepaalde. Deze classifiers kunnen de aan- of afwezigheid van diabetische voet complicaties met acceptabele performance bepalen. Echter, ze hebben te weinig capaciteit om de verschillen tussen verschillende diabetische voet complicaties te onderscheiden. Vervolg onderzoek is nodig om de prestaties van bestaande pixel classifiers te bepalen en back-end classifiers te ontwikkelen.

Voor de "thermische imaging" is een infrarood camera gebruikt om het temperatuurverschil tussen overeenkomende punten op de plantaire zijde van de linker- en de rechtervoet te vergelijken, om zo het risico op inflammatie te detecteren. Omdat 
het contrast tussen temperatuur van de voet en de omgeving laag was, was het moeilijk om het onderscheid tussen voet en omgeving te maken op basis van de thermische beelden. Om dit op te lossen werd voet segmentatie gedaan op basis van digitale kleurenfoto's. De gesegmenteerde linker- en de rechtervoet werden vervolgens geregistreerd met elkaar, zodat het verschil voor temperatuur tussen overeenkomende punten op de beide voeten kon worden vergeleken. De uitkomsten van de analyse van de thermische beelden zijn veelbelovend voor de vroege herkenning van voet complicaties bij patiënten met DM en patiënten die een risico lopen op deze complicaties. Echter, de methodologie is beperkt tot patiënten waarbij beide voeten beschikbaar zijn voor analyse.

Met "photometrische stereo imaging" is een haalbaarheidsstudie gedaan om diabetische voet complicaties te herkennen. De kromming van de gereconstrueerde oppervlakte en het gereconstrueerde oppervlakte albedo werden genomen als input kenmerken om onderscheid te maken tussen verschillende regio's op de huid aan de plantaire zijde van de voet waar diabetische voet complicaties aanwezig of afwezig waren. De resultaten wezen erop dat deze technologie mogelijkheden biedt, maar dat er nog wel beperkingen zijn om het toe te passen om diabetische voet complicaties te herkennen. Een voorbeeld hiervan is het bewegen van de voeten van patiënten tijdens de metingen. Verdere verbeteringen van deze techniek zijn nodig om dit toe te passen in een toekomstig telemedicine systeem.

De uitkomsten van de studies die in dit proefschrift zijn gepresenteerd lieten de haalbaarheid zien van de ontwikkeling van een telemedicine systeem gebaseerd op drie modaliteiten om diabetische voet complicaties te herkennen. De studies zijn een voorloper voor de ontwikkeling van een dergelijk intelligent telemedicine systeem. Verschillende mogelijke vormen van methodologie voor detectie worden voorgesteld en er wordt richting gegeven aan toekomstig onderzoek. De conclusies en aanbevelingen van dit proefschrift volgend verwachten we dat een intelligent telemedicine systeem dat metingen kan doen bij patiënten thuis of in gezondheidscentra in de toekomst ontwikkeld kan worden. 


\section{Acknowledgments}

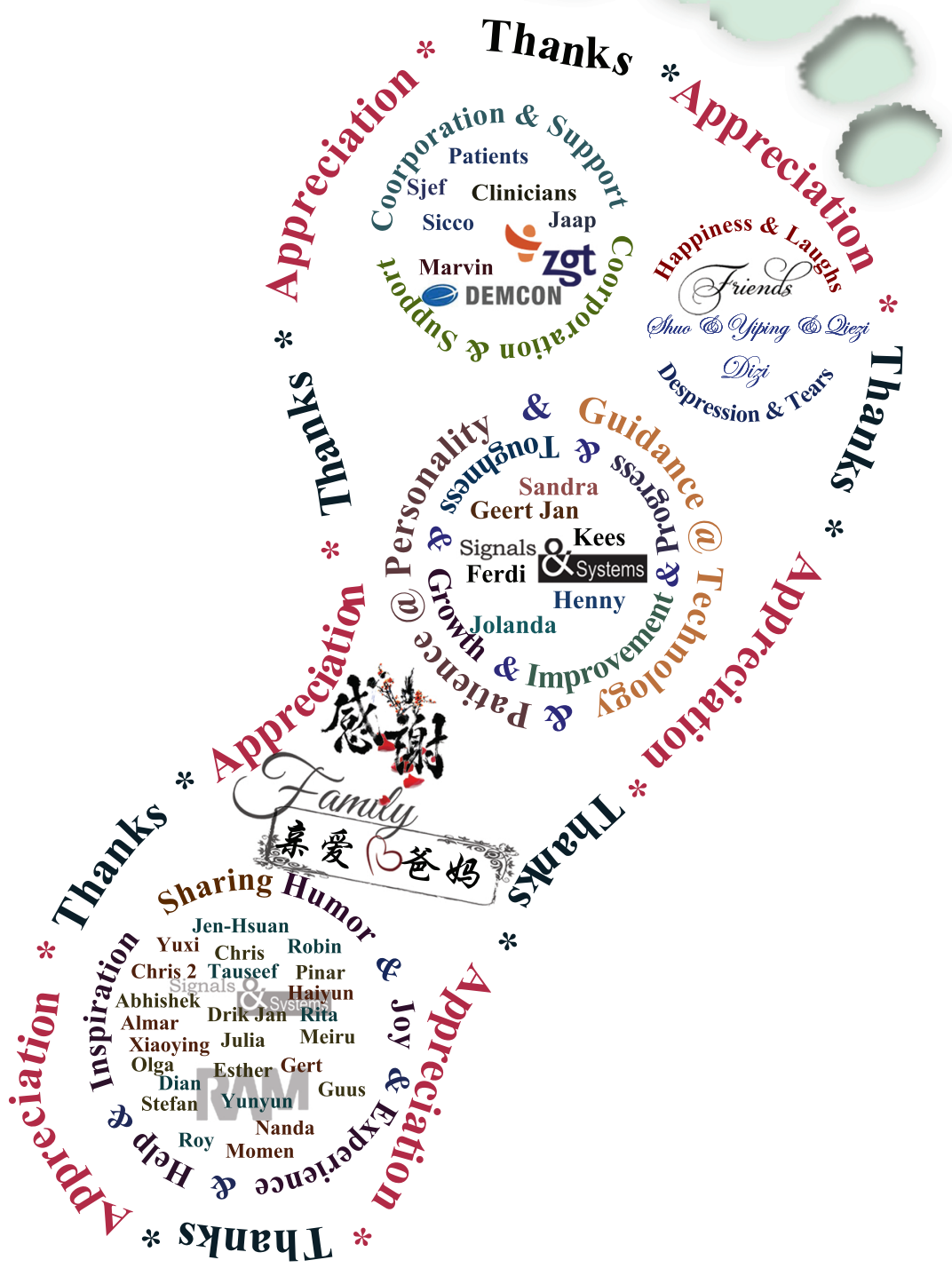

